\title{
ON MILNOR'S FIBRATION THEOREM AND ITS OFFSPRING AFTER 50 YEARS
}

\author{
JOSÉ SEADE \\ To Jack, whose profoundness and clarity of vision \\ seep into our appreciation of the beauty and depth of mathematics.
}

\begin{abstract}
Milnor's fibration theorem is about the geometry and topology of real and complex analytic maps near their critical points, a ubiquitous theme in mathematics. As such, after 50 years, this has become a whole area of research on its own, with a vast literature, plenty of different viewpoints, a large progeny, and connections with many other branches of mathematics. In this work we revisit the classical theory in both the real and complex settings, and we glance at some areas of current research and connections with other important topics. The purpose of this article is twofold. On the one hand, it should serve as an introduction to the topic for nonexperts, and on the other hand, it gives a wide perspective of some of the work on the subject that has been and is being done. It includes a vast literature for further reading.
\end{abstract}

\section{Contents}

Introduction

\section{Part I. Complex Singularities}

1. The initial thrust: Searching for homotopy spheres

2. An example: The Pham-Brieskorn singularities

3. Local conical structure of analytic sets

4. The classical fibration theorems for complex singularities

5. Extensions and refinements of Milnor's fibration theorem

6. The topology of the fibers: Milnor and Lê numbers

7. On the Milnor number

8. Indices of vector fields and the Milnor number

9. Milnor classes

10. Equisingularity

11. Relations with other branches

Received by the editors June 15, 2018.

2010 Mathematics Subject Classification. Primary 32SXX, 14BXX, 57M27, 57M50; Secondary 32QXX, 32JXX, 55S35, 57R20, 57R57, 57R77.

Key words and phrases. Milnor fibration, Milnor number, Lê numbers, vanishing cycles, equisingularity, Lipschitz, Chern classes, indices of vector fields, 3-manifolds, mixed singularities, linear actions, polar actions, homotopy spheres.

The author's research was partially supported by CONACYT 282937, PAPIIT-UNAM IN 110517, and CNRS-UMI 2001, Laboratoire Solomon Lefschetz-LaSoL, Cuernavaca, Mexico. 
Part II. Beyond the Holomorphic Realm

12. Foundations and first steps

13. On functions with a nonisolated critical point 320

14. d-regularity and Milnor fibrations 323

15. Mixed singularities

16. Linear actions, intersections of quadrics, and LVM manifolds 332

About the author $\quad 335$

Acknowledgments

References 335

\section{INTRODUCTION}

Milnor's fibration theorem in [193] is a milestone in singularity theory that has opened the way to a myriad of insights and new understandings. This is a beautiful piece of mathematics, where many different branches, aspects and ideas, come together. The theorem concerns the geometry and topology of analytic maps near their critical points.

Consider the simplest case, a holomorphic map $\left(\mathbb{C}^{n+1}, \underline{0}\right) \stackrel{f}{\rightarrow}(\mathbb{C}, 0)$ taking the origin into the origin, with an isolated critical point at $\underline{0}$. As an example one can have in mind the Pham-Brieskorn polynomials,

$$
z \mapsto z_{0}^{a_{0}}+\cdots+z_{n}^{a_{n}}, \quad a_{i} \geq 2 \text { for all } i=0,1, \ldots, n .
$$

Since $f$ is analytic, there exists $r>0$ sufficiently small so that $0 \in \mathbb{C}$ is the only critical value of the restriction $\left.f\right|_{\mathbb{B}_{r}}$, where $\mathbb{B}_{r}$ is the open ball of radius $r$ and center at $\underline{0}$. Set

$$
V:=f^{-1}(0) \text { and } \quad V^{*}:=V \backslash\{\underline{0}\} .
$$

So $V^{*}$ is an $n$-dimensional complex manifold. We know (see section 3) that $V^{*}$ meets transversally every sufficiently small sphere $\mathbb{S}_{\varepsilon}$ in $\mathbb{C}^{n+1}$ centered at $\underline{0}$ and contained in $\mathbb{B}_{r}$. The manifold $L_{V}:=V \cap \mathbb{S}_{\varepsilon}$ is called the link of the singularity, and its diffeomorphism type does not depend on the choice of the sphere. Then Milnor's theorem in 193 says that for every such sphere $\mathbb{S}_{\varepsilon}$ we have a smooth fiber bundle

$$
\varphi:=\frac{f}{|f|}: \mathbb{S}_{\varepsilon} \backslash L_{V} \longrightarrow \mathbb{S}^{1} .
$$

In fact $f$ can have nonisolated critical points. The fibers $F_{f}$ (see Figure 1) are diffeomorphic to the complex manifolds obtained by considering a regular value $t$ sufficiently near $0 \in \mathbb{C}$ and looking at the piece of $f^{-1}(t)$ contained within the open ball $\mathbb{B}_{\varepsilon}$ bounded by $\mathbb{S}_{\varepsilon}$.

Early versions of this theorem arose when Brieskorn 47] discovered that the fibration (0.2) can be used to prove that exotic spheres show up naturally as links of isolated singularities of complex hypersurfaces. In fact Brieskorn proved that every exotic sphere that bounds a parallelizable manifold is diffeomorphic to the link of a singularity as in (0.1), for appropriate $a_{i}$.

These kinds of ideas led to studying the local conical structure of all real and complex analytic sets, which we discuss in section 3 . In the rest of this paper we describe the fibration theorem for real and complex singularities, and various extensions of it. We discuss too the main ideas of its proof, and we exemplify these 


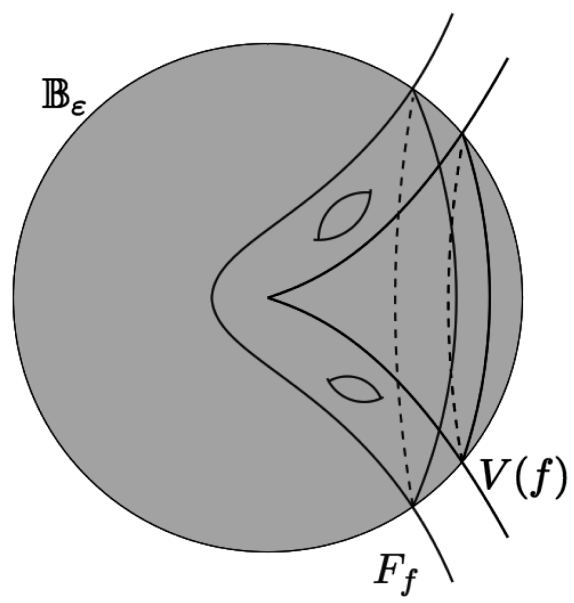

Figure 1. The Milnor fiber $F_{f}$.

with the case of the Pham-Brieskorn polynomials, where the main ingredients of the proof are shown in an elementary way.

When the map in question is holomorphic and has an isolated critical point, the fiber is diffeomorphic to a $2 n$-ball to which one attaches handles of middle index. The number of such handles is now called the Milnor number of the singularity. If the critical point is nonisolated, then the Milnor fiber is diffeomorphic to a ball to which we must attach handles of various indices. The precise number of handles of each index is prescribed by the Lê numbers of the singularity, a concept introduced by D. Massey in [175, 176].

The study of the Milnor number and its generalizations, such as the Lê numbers, has given rise to a vast literature, discussed in sections 6] to 9, In fact there are interesting relations with the theory of Chern classes for singular varieties: One has the Milnor classes, defined as the difference between the Schwartz-MacPherson and the Fulton-Johnson classes. For varieties which are complete intersections with isolated singularities, there is only one Milnor class, an integer equal to the sum of the local Milnor numbers at the singular points. So Milnor classes generalize the Milnor number to varieties which may not be complete intersections and can have nonisolated singularities.

In section 10 we glance at the important concept of equisingularity. This emerges from Zariski's seminal works [281 285], and it has important ties with Milnor's fibration theorem. The basic idea is that if $V$ is a variety with nonisolated singularities, we want to know whether the singularity of $V$ at a given point is "worse than" or "equivalent to" its singularities at other nearby points; and what does "equivalent" mean?

As mentioned above, singularity theory is a meeting point of various areas of mathematics. In section 11 we briefly discuss relations of complex singularities with open-book decompositions and fibered knots, contact structures, and low dimensional manifolds. Later we discuss relations with the so-called moment-angle manifolds, which spring from mathematical physics. For this we need to extend the discussion beyond the holomorphic realm. 
Milnor proved in 192, 193 that the fibration theorem also holds for analytic maps $\left(\mathbb{R}^{n}, \underline{0}\right) \stackrel{f}{\rightarrow}\left(\mathbb{R}^{p}, 0\right), n \geq p \geq 1$, with an isolated critical point, and one has a fiber bundle

$$
\varphi: \mathbb{S}_{\varepsilon} \backslash L_{V} \longrightarrow \mathbb{S}^{1}
$$

Yet, in general the projection map $\varphi$ can only be taken as $f /|f|$ in a neighborhood of the link $L_{V}$. Also, as pointed out in [193, Chapter 11], the condition of having an isolated critical point is very stringent. Generically, the set of critical values has positive dimension, and even if the only critical value is 0 , it is fairly stringent to ask to have an isolated critical point. In sections 12 to 14 we discuss Milnor fibrations for real analytic maps in general, starting with the isolated singularity case. We discuss a regularity condition that is necessary and sufficient to ensure that if we have the fibration (0.3), we can take the projection $\varphi$ to be $f /|f|$ everywhere. This is called d-regularity.

We look also at two particularly interesting classes of maps: the meromorphic germs and maps of the form $f \bar{g}$ with $f, g$ holomorphic. Notice that away from the set $f g=0$, one has

$$
\frac{f / g}{|f / g|}=\frac{f \bar{g}}{|f \bar{g}|}
$$

The study of Milnor fibrations for meromorphic germs began with a couple of papers [125, 126] by Gusein-Zade et al. (see also [33, 34]). The study of Milnor fibrations and open-books defined by functions $f \bar{g}$ essentially began in [235, 237], though this type of map had already appeared in $[1,130,193$. We study these in a section under the name "Mixed singularities", which by definition are critical points of analytic functions in the complex variables $z_{1}, \ldots, z_{n}$ and their conjugates.

Polar weighted homogeneous singularities are a particularly interesting class of mixed functions which are reminiscent of the classical weighted homogeneous polynomials. Now one has a weighted action of $\mathbb{S}^{1} \times \mathbb{R}^{+} \cong \mathbb{C}^{*}$ on $\mathbb{C}^{n}$, and the function brings out scalars to some power. Unlike the classical case of weighted homogeneous functions, now $\mathbb{S}^{1}$ and $\mathbb{R}^{+}$can act with different weights. The paradigm examples of such singularities are the twisted Pham-Brieskorn polynomials, studied in $[241,248,249$ :

$$
\left(z_{1}, \ldots, z_{n}\right) \longrightarrow z_{1}^{a_{1}} \bar{z}_{\sigma(1)}+\cdots+z_{n}^{a_{n}} \bar{z}_{\sigma(n)}, \quad a_{i} \geq 2
$$

where $\sigma$ is a permutation of the set $\{1, \ldots, n\}$. If $\sigma$ is the identity, these are essentially equivalent to usual Pham-Brieskorn singularities (by [222,241]).

The notions of weighted polar actions and polar singularities were introduced in [62], inspired by [241]. The name mixed singularity was coined by M. Oka [219], and his vast contributions to the subject have turned this into a whole new area of research. These singularities also provide interesting open-book decompositions similar to Milnor's open-books but which do not appear in complex singularities (see for instance [5, 7, 236]).

We finish this article with an especially interesting class of mixed singularities that spring from the study of holomorphic linear actions of $\mathbb{C}^{m}$ in $\mathbb{C}^{n}, 0<m \ll n$. Under appropriate conditions, there is an open dense set in $\mathbb{C}^{n}$ of points that belong to a type of orbits called "Siegel leaves". These are parameterized by a $C^{\infty}$-manifold $V^{*}:=V \backslash\{\underline{0}\}$, where $V$ is a complete intersection defined by $2 m$ real-valued quadratic polynomial equations: $V^{*}$ consists of the points where the orbits are tangent to the foliation in $\mathbb{C}^{n} \backslash\{0\}$ given by all spheres centered at the 
origin. The manifold $V^{*}$ has a canonical complex structure that comes from being everywhere transversal to the orbits of the $\mathbb{C}^{m}$-action, and it has a canonical $\mathbb{C}^{*}$ action with compact quotient $V^{*} / \mathbb{C}^{*}$. These quotients are known as $L V M$-manifolds (for López de Madrano, Verjovsky, and Meersseman); these have a rich geometry and topology, and they are a class of the so-called moment-angle manifolds, of relevance in mathematical physics.

We begin this article by pointing out important relations between complex singularities and exotic spheres, and we close it with an important relation between real singularities and moment-angle manifolds: this exemplifies the strong interactions singularity theory has with other areas of mathematics.

My aim with this article is to share with the readers some of the beauty and richness of singularity theory, a fascinating area of mathematics which somehow begins with Isaac Newton and is built upon the foundational work of Hassler Whitney, René Thom, John Milnor, Oscar Zariski, Heisuke Hironaka, Egbert Brieskorn, Vladimir Arnold, and many others. Arnold used to say [18] that the main goal in most problems of singularity theory is to understand the qualitative change in objects that depend on parameters, and which may come from analysis, geometry, physics, or from some other science. Milnor's fibration theorem certainly fits within this framework.

\section{Part I. Complex Singularities}

\section{The initial thrust: Searching for homotopy Spheres}

In 1956 John Willard Milnor (b. 1931) surprised the world by finding [191 the first "exotic spheres": seven-dimensional smooth manifolds homeomorphic to $\mathbb{S}^{7}$ but with nonequivalent differentiable structures.

The set of equivalence classes of smooth structures on the $n$-sphere $\mathbb{S}^{n}$ is a monoid where the operation is the connected sum. For $n \neq 4$ this monoid is a group and it is isomorphic to the finite abelian group $\Theta_{n}$ of h-cobordism classes of oriented homotopy $n$-spheres, with the connected sum as operation; the identity element is the standard $\mathbb{S}^{n}$. This group was studied by Kervaire and Milnor in [140] for $n \geq 5$. They noticed that $\Theta_{n}$ contains a "preferred subgroup", denoted $b P_{n+1} \subset \Theta_{n}$, of those homotopy spheres that bound a parallelizable manifold, i.e., a manifold with trivial tangent bundle. For $n \neq 3$ odd, this is a finite cyclic group which has finite index in $\Theta_{n}$. This cyclic group has order 1 or 2 for $n \equiv 1(\bmod 4)$, but for $n \equiv 3(\bmod 4)$ its order $\left|b P_{4 m}\right|$ grows more than exponentially:

$$
\left|b P_{4 m}\right|=\left[2^{2 m-2}\left(2^{2 m-1}-1\right)\right] \cdot\left[\text { numerator of }\left(\frac{4 \mathcal{B}_{m}}{m}\right)\right],
$$

where the $\mathcal{B}_{m}$ are the Bernoulli numbers. Thus for instance (see [133, 140]), for $n=7,11,15$, or 19 there are, respectively, $\left|b P_{n+1}\right|=28,992,8128$, and 130816 nonequivalent differentiable structures on the $n$-sphere that bound a parallelizable manifold.

A question was, How do we construct those exotic spheres? An approach to this problem is by looking at the links of isolated complex singularities. This led to Milnor's fibration theorem, as we now explain.

Let $(V, P)$ be a complex analytic variety of complex dimension $n$ in some affine space $\mathbb{C}^{N}$, with a unique singular point at $P$. Then $V^{*}=V \backslash\{P\}$ is a complex $n$-manifold. One has the following. 
Proposition 1.1. There exists $\varepsilon>0$ sufficiently small, so that every sphere $\mathbb{S}_{r}$ in $\mathbb{C}^{N}$ of radius $r \leq \varepsilon$ and center at $P$ meets $V^{*}$ transversally.

This is proved in [193, Chapter 2] when $X$ is algebraic and it is a particular case of a general theorem about the local conical structure of analytic sets; see section 3. It follows that if $(V, P)$ is as above, then its link $L_{V}:=\mathbb{S}_{\varepsilon} \cap V$ is a smooth real analytic manifold of dimension $2 n-1$.

Question 1.2. Can we know when $L_{V}$ is a homotopy sphere? And if so, can we determine which element in $\Theta_{n}$ it represents?

For $n=1$, the question is trivial since $L_{V}$ is a union of circles, one for each branch of $V$. For $n=2$, if $V$ has a normal singularity at $P$, then its link is never simply connected; see 199. When $V$ is a complex hypersurface (i.e., defined by one single equation), Question 1.2 was answered by the work of various people in the 1960s, most notably by E. Brieskorn, F. Hirzebruch, and J. Milnor; see [4, 119, 134, 193.

For instance, in dimension 7 we know from 140 that there exist 28 classes of homotopy spheres including the standard one, and all of them bound a parallelizable manifold. Brieskorn proved in [47] that all these 28 classes can be represented by the link $L_{V}$ of some hypersurface in $\mathbb{C}^{5}$ of the form

$$
z_{0}^{a_{0}}+z_{1}^{a_{1}}+\cdots+z_{4}^{a_{4}}=0 .
$$

The fibration theorem is the culmination of a series of works by various authors, starting with F. Pham 232.

Let $V$ be the zero-locus of an analytic map $\left(\mathbb{C}^{n+1}, \underline{0}\right) \stackrel{f}{\rightarrow}(\mathbb{C}, 0)$ with an isolated critical point at $\underline{0}$. Equip its link $L_{V}^{2 n-1}$ with its natural differentiable structure as the transverse intersection $L_{V}=\mathbb{S}_{\varepsilon} \cap V$ of two smooth submanifolds of $\mathbb{C}^{n+1}$. One has a map

$$
\varphi:=\frac{f}{|f|}: \mathbb{S}_{\varepsilon} \backslash L_{V} \longrightarrow \mathbb{S}^{1},
$$

and Milnor's fibration theorem says that this is a smooth fiber bundle.

Milnor also proves that the fiber $F_{t}$ is diffeomorphic to the portion of a noncritical level $f^{-1}(t)$ contained within the ball $\mathbb{B}_{\varepsilon}$ bounded by $\mathbb{S}_{\varepsilon}$; see Figure 2 , This implies that the normal bundle of $F_{t}$ is trivial, being the inverse image of a regular value. Hence the tangent bundle $T F_{t}$ is stably trivial, i.e., $F_{t}$ is stably parallelizable, and we know from [140] that for compact connected manifolds with nonempty boundary, stably parallelizable implies parallelizable. Thus we get the following.

Proposition 1.4. The link $L_{V}$ of every complex hypersurface isolated singularity bounds the fibers $F_{\theta}$, which are parallelizable manifolds.

The point is to know when $L_{V}$ is a homotopy sphere, and when this happens, which element it represents in $b P_{2 n}$. For this, Milnor proved the following in [193].

Proposition 1.5. The link of every isolated hypersurface singularity in $\mathbb{C}^{n+1}$ is $(n-2)$-connected, and the fiber $F_{t}$ has the homotopy of a bouquet $\bigvee \mathbb{S}^{n}$ of spheres of middle dimension.

The number of spheres in the bouquet $\bigvee \mathbb{S}^{n}$ is strictly positive, unless $V$ has no singularity. This is now called the Milnor number $\mu$ of $f$ (see section 6).

For $n>2$, the link is simply connected, and therefore the Hurewicz isomorphism implies that the homology of $L_{V}$ also vanishes in dimensions $i=1, \ldots, n-2$. Since 
$L_{V}$ is always orientable, by the Poincaré duality isomorphism its homology vanishes in dimensions $n+i, i=1, \ldots, n-2$ as well. Thus the only possibly nonzero groups are in dimensions $i=n, n-1$ and of course $i=0,2 n-1$ where they are isomorphic to the group of the integers (or the corresponding ring of coefficients).

If $H_{n-1}\left(L_{V}\right)$ vanishes, then $H_{n}\left(L_{V}\right)$ also vanishes by duality, and $L_{V}$ is a homology sphere. If $n \geq 2$, then $L_{V}$ is simply connected by 193 . Hence, if $n>2$, then Smale's theorem in 256] implies (Poincaré's Conjecture in dimensions $\geq 5$ ) that $L_{V}$ is actually homeomorphic to $\mathbb{S}^{2 n-1}$. So the question is to decide when $H_{n-1}\left(L_{V}\right)$ vanishes, and it is here that the fibration theorem enters the scene: fix a fiber $F_{0}$ and notice that $F_{0}$ and its complement in $\mathbb{S}_{\varepsilon} \backslash L_{V}$ have the same homotopy type. Consider the monodromy $h$ (a first return map) of the bundle (1.3) and the induced representation in the middle homology of the fiber

$$
h_{*}: H_{n}\left(F_{0}\right) \rightarrow H_{n}\left(F_{0}\right) .
$$

One has the corresponding Wang sequence (see [193, p. 68])

$$
H_{n}\left(F_{0}\right) \stackrel{h_{*}-I_{*}}{\longrightarrow} H_{n}\left(F_{0}\right) \longrightarrow H_{n}\left(\mathbb{S}_{\varepsilon}-L_{V}\right) \longrightarrow 0 .
$$

Using this, one arrives at Milnor's theorem [193, Theorem 8.5], that for $n>2$ the link is a topological sphere if and only if the determinant of $h_{*}-I_{*}$ is \pm 1 , which was already proved by F. Pham in 232 for the Pham-Brieskorn polynomials. Using this, for instance Hirzebruch proved in [134, pp. 20-21] that the links of the following singularities are all homotopy spheres:

$$
z_{0}^{3}+z_{1}^{6 k-1}+z_{2}^{2}+\cdots+z_{2 m}^{2}=0 ; \quad k \geq 1, m \geq 2 .
$$

One has the following remarkable theorm of Brieskorn [47, Korollar 2].

Theorem 1.6. Every exotic sphere of dimension $m=2 n-1>6$ that bounds a parallelizable manifold is the link of some hypersurface singularity of the form

$$
z_{0}^{a_{0}}+z_{1}^{a_{1}}+\cdots+z_{n}^{a_{n}}=0,
$$

for some appropriate integers $a_{i} \geq 2, i=0,1, \ldots, n$.

One may consider singularities which are not hypersurfaces and try to produce other elements in the homotopy of spheres. To my knowledge, little is known about this problem. If we consider complex isolated complete intersection singularities, one always has a Milnor fibration and the fibers can be regarded as being the interior of compact parallelizable manifolds with the link as its boundary, by [128]. So in these cases, if the link is an exotic sphere, this is in $b P_{2 n} \subset \Theta_{2 n-1}$, which is the simplest and best understood part of $\Theta_{2 n-1}$.

I thank Patrick Popescu-Pampu for bringing to my attention the following interesting question posed by A. Durfee in [277, Problem H, p. 252]:

Question 1.7. Does every exotic sphere occur as the link of an isolated complex singularity?

A step for answering Question 1.7 is the question that Popescu-Pampu originally asked me:

Question 1.8. Does there exist a complex isolated singularity whose link is a homotopy sphere that does not bound a parallelizable manifold?

Such examples, if they exist, would produce elements in the most mysterious part of the groups $\Theta_{n}$. 


\section{An example: The Pham-Brieskorn singularities}

Consider a Pham-Brieskorn polynomial $f: \mathbb{C}^{n} \rightarrow \mathbb{C}$,

$$
\left(z_{1}, \ldots, z_{n}\right) \stackrel{f}{\mapsto} z_{1}^{a_{1}}+\cdots+z_{n}^{a_{n}}, \quad a_{i} \geq 2 .
$$

The origin $\underline{0} \in \mathbb{C}^{n}$ is its only critical point, so $V:=f^{-1}(0)$ is a complex hypersurface with an isolated singularity at $\underline{0}$. Let $d$ be the lowest common multiple of the $a_{i}$ and for each $i=1, \ldots, n$ set $d_{i}=d / a_{i}$. Then for every nonzero complex number $\lambda \in \mathbb{C}^{*}$ one has a $\mathbb{C}^{*}$-action on $\mathbb{C}^{n}$ determined by

$$
\lambda \cdot\left(z_{1}, \ldots, z_{n}\right) \mapsto\left(\lambda^{d_{1}} z_{1}, \ldots, \lambda^{d_{n}} z_{n}\right) .
$$

Observe that one has

$$
f\left(\lambda^{d_{1}} z_{1}, \ldots, \lambda^{d_{n}} z_{n}\right)=\lambda^{d} f\left(z_{1}, \ldots, z_{n}\right),
$$

so $f$ is weighted homogeneous. This $\mathbb{C}^{*}$-action has $\underline{0}$ as its only fixed point and $V$ is an invariant set, union of $\mathbb{C}^{*}$-orbits. This has the following properties.

Property 1. Restricting the action to the positive real numbers $t \in \mathbb{R}^{+}$, we get a flow such that the following hold.

- Each orbit converges to $\underline{0}$ as $t$ tends to 0 , and it goes to infinity as $t$ tends to $\infty$.

- Each orbit is transversal to all spheres centered at $\underline{0}$. Hence $V$ intersects transversally every $(2 n-1)$-sphere $\mathbb{S}_{r}$ centered at $\underline{0}$, so $K_{r}:=V \cap \mathbb{S}_{r}$ is a real codimension 2 smooth submanifold of $\mathbb{S}_{r}$.

- Given arbitrary spheres $\mathbb{S}_{r}, \mathbb{S}_{r^{\prime}}$ centered at $\underline{0}$, the flow gives a diffeomorphism from $\mathbb{S}_{r}$ into $\mathbb{S}_{r^{\prime}}$ taking $K_{r}$ into $K_{r^{\prime}}$. Moreover, the flow determines a one-parameter group of diffeomorphisms that exhibits the pair $\left(\mathbb{C}^{n}, V\right)$ as the cone over $\left(\mathbb{S}_{r}, K_{r}\right)$. We denote the manifold $K_{r}$ by $L_{f}$ and call it the link (see 77, 193]).

Property 2. The argument of the complex number $f(z)$ is constant on each orbit of the above flow, i.e., $f(z) /|f(z)|=f(t z) /|f(t z)|$ for all $t \in \mathbb{R}^{+}$.

Property 3. The restriction of the $\mathbb{C}^{*}$-action to $\mathbb{S}^{1}$ leaves invariant every sphere around $\underline{0}$. Multiplication by $e^{i \theta}$ in $\mathbb{C}^{n}$ transports each fiber $f^{-1}(\zeta)$ into the fiber $f^{-1}\left(e^{i \theta \bar{d}} \cdot \zeta\right)$. Hence $\mathbb{S}^{1}$ acts on each tube $N(\delta):=f^{-1}\left(\partial \mathbb{D}_{\delta}\right)$, where $\partial \mathbb{D}_{\delta} \cong \mathbb{S}^{1}$ is the boundary of the disc in $\mathbb{C}$ of radius $\delta>0$ and centered at 0 . A direct computation shows that the orbits of this action are transverse to the fibers of $f$. So we have a smooth fiber bundle, $N(\delta) \stackrel{f}{\rightarrow} \partial \mathbb{D}_{\delta}$.

Now observe that for each line $\mathcal{L}_{\theta}$ through the origin in $\mathbb{C}$, we may consider the set

$$
X_{\theta}:=\left\{z \in \mathbb{C}^{n} \mid f(z) \in \mathcal{L}_{\theta}\right\} .
$$

Each $X_{\theta}$ is a real analytic hypersurface with an isolated singularity at $\underline{0}$, their union fills the entire $\mathbb{C}^{n}$, and their intersection is $V$. By Property 1 each $X_{\theta}$ is transversal to all the spheres, and by Property 3 , the $\mathbb{S}^{1}$-action permutes these hypersurfaces. Thus one has the following.

Property 4. These varieties define a pencil in $\mathbb{C}^{n}$, a sort of open-book where the binding is now the singular variety $V$, and each of these varieties is transverse to 


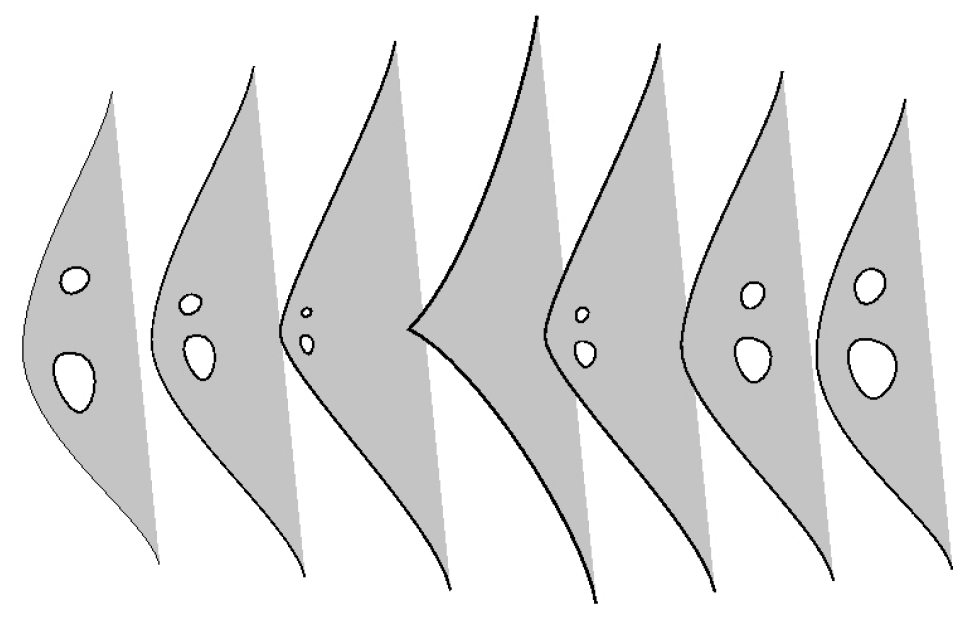

FiguRE 2. The noncritical levels are all diffeomorphic.

every sphere around $\underline{0}$. If we remove $V$ from $\mathbb{C}^{n}$, then for every ball around $\underline{0}$ we get a fiber bundle

$$
\varphi=\frac{f}{|f|}: \mathbb{B}^{2 n} \backslash V \longrightarrow \mathbb{S}^{1}
$$

The fiber over a point $e^{i \theta}$ is a connected component of $X_{\theta} \backslash V$. The other component is $f^{-1}\left(e^{-i \theta}\right)$.

Property 5. We now focus our attention near the origin, say restricted to the unit ball $\mathbb{B}^{2 n}$ in $\mathbb{C}^{n}$. Since each $X_{\theta}$ meets transversally the sphere $\mathbb{S}^{2 n-1}=\partial \mathbb{B}^{2 n}$, the intersection is a smooth codimension 1 submanifold of the sphere, containing the link $L_{f}=V \cap \mathbb{S}^{2 n-1}$. And since the orbits of the $\mathbb{S}^{1}$-action preserve the sphere $\mathbb{S}^{2 n-1}$, the restriction of $\varphi$ to $\mathbb{S}^{2 n-1}$ defines the classical Milnor fibration

$$
\varphi=\frac{f}{|f|}: \mathbb{S}^{2 n-1} \backslash L_{f} \longrightarrow \mathbb{S}^{1},
$$

Property 6. Since $V$ is transverse to the unit sphere $\mathbb{S}^{2 n-1}$ and each point in $V \backslash\{\underline{0}\}$ is regular, each fiber $f^{-1}(t)$ with $|t|$ sufficiently small is also transverse to $\mathbb{S}^{2 n-1}$. Hence, if $N(\delta)$ is as in Property 3 and we set $N(1, \delta):=N(\delta) \cap \mathbb{B}^{2 n}$, where the 1 means that the ball $\mathbb{B}^{2 n}$ has radius 1 , we have that the fiber bundle described by Property 3 determines a fiber bundle

$$
f: N(1, \delta) \rightarrow \partial \mathbb{D}_{\delta} \cong \mathbb{S}^{1}
$$

This is the second classical version of Milnor's fibration for the map $f$; see Figure 2

Property 7. Notice that by Property 2 , each $\mathbb{R}^{+}$-orbit is everywhere transverse to the tube $N(\delta)$ and transverse to the sphere $\mathbb{S}^{2 n-1}$, and the complex numbers $f(z)$ have constant argument along each orbit. Thence the integral lines of this action determine a diffeomorphism between $N(1, d)$ and $\mathbb{S}^{2 n-1}$ minus the part of the sphere contained inside the open solid tube $f^{-1}\left(\mathbb{D}_{\delta}\right)$. This determines the classical equivalence between the Milnor fibration in the sphere (4.1) and the Milnor-Lê fibration in the tube (4.2). 
We now remark that everything we said above works in exactly the same way for all weighted homogeneous complex singularities; i.e., for all complex polynomials $f$ for which there is a $\mathbb{C}^{*}$-action on $\mathbb{C}^{n}$ as above, for some positive integers $\left\{d ; d_{1}, \ldots, d_{n}\right\}$,

$$
\lambda \cdot\left(z_{1}, \ldots, z_{n}\right)=\left(\lambda^{d_{1}} z_{1}, \ldots, \lambda^{d_{n}} z_{n}\right),
$$

satisfying that for all $\lambda \in \mathbb{C}^{*}$ and for all $z \in \mathbb{C}^{n}$, one has

$$
f(\lambda \cdot z)=\lambda^{d} \cdot f(z) .
$$

These all have the same Properties 1 to 7 . As we will see in what follows, all real analytic isolated singularities can be equipped with flows that satisfy properties analogous to 1 and 3 to 6 , but not always 2 . This implies that we have a fibration as in (2.3) and it can be carried to a fibration on the sphere as in (2.2) but the projection map $\varphi$ may not always be taken to be $f /|f|$ away from a neighborhood of the link. Also, having Property 2 grants that $\varphi$ can be taken as $f /|f|$ everywhere, and this is equivalent to the map-germ being d-regular, a concept that we discuss in section 14

\section{LOCAL CONICAL STRUCTURE OF ANALYTIC SETS}

Consider a reduced, equidimensional real analytic space $V$ of dimension $n$, defined in an open ball $\mathbb{B}_{r}(\underline{0}) \subset \mathbb{R}^{N}$ around the origin. Assume $V$ contains the origin $\underline{0}$ and $V^{*}:=V-\{\underline{0}\}$ is a real analytic manifold of dimension $n>0$. The following is proved in [193], and it can be deduced from [168].

Theorem 3.1 (Milnor 1968). There exists $\varepsilon>0$ sufficiently small, so that every sphere in $\mathbb{R}^{N}$ centered at $\underline{0}$ and with radius $\leq \varepsilon$ intersects $V^{*}$ transversally. Moreover, there is a smooth one-parameter family of diffeomorphisms $\left\{\gamma_{t}\right\}, t \in[0, \varepsilon)$, such that $\gamma_{0}$ is the identity, and if $\mathbb{S}_{\varepsilon-t}$ denotes the sphere of radius $\varepsilon-t$, then each $\gamma_{t}$ carries the pair $\left(\mathbb{S}_{\varepsilon}, \mathbb{S}_{\varepsilon} \cap V\right)$ into $\left(\mathbb{S}_{\varepsilon-t}, \mathbb{S}_{\varepsilon-t} \cap V\right)$. The pair $\left(\mathbb{B}_{\varepsilon}, \mathbb{B}_{\varepsilon} \cap V\right)$ is homeomorphic to the cone over $\left(\mathbb{S}_{\varepsilon}, \mathbb{S}_{\varepsilon} \cap V\right)$.

The idea of the proof is simple: Consider the function $\mathbb{R}^{N} \stackrel{d}{\rightarrow} \mathbb{R}$ given by $d\left(x_{1}, \ldots, x_{N}\right)=x_{1}^{2}+\cdots+x_{N}^{2}$, so that $d$ is the square of the function "distance to

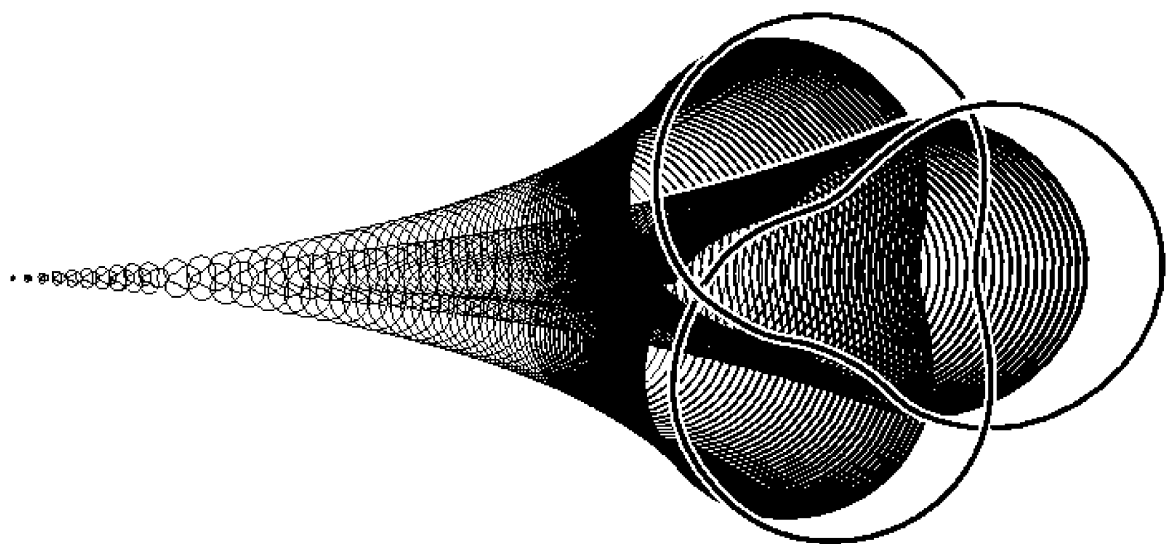

FiguRE 3. The link of the singularity determines the topological type. 
$\underline{0}$ ". The solutions of its gradient vector field $\nabla d$ are the straight rays that emanate from the origin. Let us adapt this vector field to $V$. For this, take the restriction $d_{V}$ of $d$ to $V$. At each point $x \in V^{*}$ the gradient vector $\nabla d_{V}(x)$ is obtained by projecting $\nabla d(x)$ to $T_{x} V^{*}$, the tangent space of $V^{*}$ at $x$, so $\nabla d_{V}(x)$ vanishes if and only if $T_{x} V^{*} \subset T \mathbb{S}_{x}$. This means that a point $x \in V^{*}$ is a critical point of $d_{V}$ iff $V^{*}$ is tangent at $x$ to the sphere passing through $x$ and centered at $\underline{0}$. Just as in [193, Corollary 2.8], one has that $d_{V}$ has at most a finite number of critical values corresponding to points in $V^{*}$, since it is the restriction of an analytic function on $\mathbb{B}_{r}(\underline{0})$. Hence $V^{*}$ meets transversally all sufficiently small spheres around the origin in $\mathbb{R}^{N}$. The gradient vector field of $d_{V}$ is now everywhere transversal to the spheres around $\underline{0}$, and it can be assumed to be integrable. Hence it defines a one-parameter family of local diffeomorphisms of $V^{*}$ taking each link into "smaller" links, proving Theorem 3.1

Theorem 3.1 was extended in 51 to varieties with arbitrary singular locus using Whitney stratifications (we refer to [71,116] for background material on stratifications). A more refined argument due to A. Durfee [77] (see also [159]) and based on the Curve Selection Lemma of [193], shows that in fact the diffeomorphism type of the manifold $V \cap \mathbb{S}_{\varepsilon}$ is also independent of the choice of the embedding of $V$ in $\mathbb{R}^{N}$. One has the following.

Theorem 3.2. Let $V$ be a real or complex analytic set in $\mathbb{R}^{m}$, and let $P$ be a singular point in $V$. Then there exists a Whitney stratification of $\mathbb{R}^{m}$ for which $V$ is a union of strata, $P$ is a point stratum, and one has the following (see Figure 3).

(1) There exists $\varepsilon>0$ sufficiently small, so that every sphere $\mathbb{S}_{r}$ in $\mathbb{R}^{m}$ of radius $r \leq \varepsilon$ and center at $P$ meets transversally at every stratum in $V$.

(2) One has a homeomorphism of pairs: $\left(\mathbb{B}_{\varepsilon}, \mathbb{B}_{\varepsilon} \cap V\right) \cong$ Cone $\left(\mathbb{S}_{\varepsilon}, \mathbb{S}_{\varepsilon} \cap V\right)$.

(3) The homeomorphism type of $L_{V}:=\mathbb{S}_{\varepsilon} \cap V$ is independent of the choice of the defining equations for $V$.

Definition 3.3. The manifold $L_{V}:=V \cap \mathbb{S}_{\varepsilon}$ is called the link of $V$ at 0 , and a sphere $\mathbb{S}_{\varepsilon}$ as in Theorem 3.2 is called a Milnor sphere for $V$. We also denote $L_{V}$ by $L_{f}$ when we want to emphasize the function rather than the space $V$.

\section{The Classical Fibration theOrems For COMPleX Singularities}

The first version of Milnor's fibration theorem says the following.

Theorem 4.1 (Fibration theorem, first version). Let $U$ be an open neighborhood of the origin $\underline{0} \in \mathbb{C}^{n+1}$, and let $f:(U, \underline{0}) \rightarrow(\mathbb{C}, 0)$ be a complex analytic map. Set $V:=f^{-1}(0)$ and $L_{V}:=V \cap \mathbb{S}_{\varepsilon}$, where $\mathbb{S}_{\varepsilon}$ is a sufficiently small sphere in $U$ centered at $\underline{0}$. Then

is a $C^{\infty}$ fiber bundle.

$$
\varphi:=\frac{f}{|f|}: \mathbb{S}_{\varepsilon} \backslash L_{V} \longrightarrow \mathbb{S}^{1}
$$

The proof by Milnor in 193 . uses the Curve Selection Lemma first to show that the map $\varphi$ has no critical points and then to construct an appropriate vector field on $\mathbb{S}_{\varepsilon} \backslash L_{V}$ that shows the local triviality, i.e., that each fiber of $\varphi$ has a neighborhood which is a product. Nowadays, the most common proof of Theorem 4.1 follows the original approach sketched by Milnor himself in a previously unpublished article 192. The starting point is the following. 
Theorem 4.2 (Fibration theorem, second version). With the above hypothesis and notation, let $\delta>0$ be sufficiently small with respect to $\varepsilon$ so that for every $t \in \mathbb{C}$ with $|t| \leq \delta$ the fiber $f^{-1}(t)$ meets the sphere $\mathbb{S}_{\varepsilon}$ transversally. Let $\mathbb{D}_{\delta}$ be the disc in $\mathbb{C}$ of radius $\delta$ and center at 0 ; let $\partial \mathbb{D}_{\delta} \cong \mathbb{S}^{1}$ be its boundary and set $N(e, \delta):=$ $f^{-1}\left(\partial \mathbb{D}_{\delta}\right) \cap \mathbb{B}_{\varepsilon}$, where $\mathbb{B}_{\varepsilon}$ is the open ball in $\mathbb{C}^{n+1}$ bounded by $\mathbb{S}_{\varepsilon}$. Then,

$$
f_{\left.\right|_{N(e, \delta)}}: N(e, \delta) \longrightarrow \partial \mathbb{D}_{\delta}
$$

is a $C^{\infty}$ fiber bundle (essentially) isomorphic to that in Theorem 4.1 .

The word "essentially" in the last statement is because the fibers in 4.2 are compact, while those in Theorem 4.1 are open manifolds. To have an actual isomorphism of the two fibrations, one must restrict the fibration in 4.2 to the open ball.

Milnor proved this theorem in 192 when the map-germ $f$ has an isolated critical point. In the general case, Milnor proved in [193] that the fibers in Theorem 4.2 (restricted to the open ball) are diffeomorphic to those in Theorem 4.1. In order to prove that one actually has a fiber bundle in Theorem 4.2 one must grant that given $\varepsilon>0$ as above, there exists a $\delta$ as stated, such that all fibers $f^{-1}(t)$ with $|t| \leq \delta$ meet the sphere $\mathbb{S}_{\varepsilon}$ transversally. This was not known until 1977 when Hironaka proved in 131 that all complex-valued holomorphic maps have a Thom stratification.

To prove Theorem 4.2, we restrict $f$ to a sufficiently small open ball $\mathbb{B}_{r}$ around $\underline{0}$ so that $0 \in \mathbb{C}$ is its only critical value. We equip $\mathbb{B}_{r}$ with a Thom stratification [131, so that $V$ is a union of strata, and we assume that $\underline{0}$ itself is a stratum. Now let $\mathbb{S}_{\varepsilon} \subset \mathbb{B}_{r}$ be a Milnor sphere for $f$, so that every sphere of radius $\leq r$ meets transversally each stratum in $V$; this is possible by Theorem 3.2 . By compactness, this implies that there exists $\delta>0$ such that for each $t \in \mathbb{C}$ with $0<|t| \leq \delta$, the fiber $f^{-1}(t)$ meets $\mathbb{S}_{\varepsilon}$ transversally. Hence all fibers in Theorem 4.2 are compact smooth manifolds with boundary. The proof of the local triviality is as in the usual proof of Ehresmann's fibration lemma - lifting via the Jacobian of $f$ the appropriate vector fields in $\mathbb{C}$ to vector fields in $\mathbb{B}_{\varepsilon}$ which are normal to the fiber. The only additional thing is that we must choose the liftings so that the vector fields are also tangent to $\mathbb{S}_{\varepsilon}$, which is possible because the fiber is transversal to the sphere (see [249, 279]).

The next step to prove Theorem 4.1 is implicit in 193: we inflate the Milnor tube, carrying the fibration in the tube into the fibration in the sphere as stated (see Figure 44). This relies on the Curve Selection Lemma. The key for this is constructing a vector field as stated in the following lemma.

Lemma 4.3. There exists an integrable vector field $\xi$ on $\mathbb{B}_{\varepsilon} \backslash V$ such that the following hold.

(1) Its integral lines are transversal to all Milnor tubes $f^{-1}\left(\mathbb{S}_{r}^{1}\right)$.

(2) Its integral lines are transversal to all spheres centered at $\underline{0}$.

(3) Its integral lines travel along points where $f$ has constant argument. That is, if $z, w$ are points in $\mathbb{B}_{\varepsilon} \backslash V$ which are in the same integral line of $\xi$, then $f(z) /|f(z)|=f(w) /|f(w)|$.

We remark that constructing a vector field that satisfies the first two conditions is easy and can also be done in the real analytic category. This allows one to inflate 


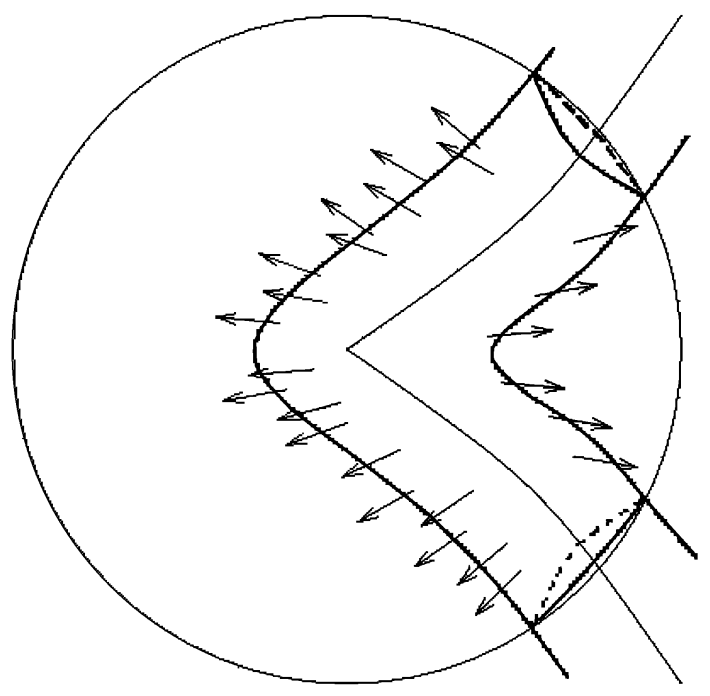

Figure 4. The vector field that carries a Milnor tube into the sphere.

the tube to the sphere so that we get a homeomorphism

$$
h: N(\varepsilon, \eta) \longrightarrow \overline{\mathbb{S}_{\varepsilon} \backslash N(\varepsilon, \eta)}
$$

in the obvious way: for each $z \in N(\varepsilon, \eta)$, we consider the unique integral line of $\xi$ passing by $z$; and we then travel along this integral line until it hits the sphere $\mathbb{S}_{\varepsilon}$. We thus get a fiber bundle $\varphi: \mathbb{S}_{\varepsilon} \backslash L_{V} \rightarrow \mathbb{S}^{1}$ with projection map $\varphi:=f \circ h^{-1}$. The hard part is having one such vector field that further satisfies the third condition. This grants that the projection map in Theorem 4.1 can be taken as $\varphi=f /|f|$ and the two fibrations are equivalent.

Having these two equivalent fiber bundles associated to a map-germ brings great richness. The first fibration is interesting for topology and differential geometry. This has important relations with knot theory, open-book decompositions, and contact and symplectic geometry. The second fibration lends itself more naturally to generalizations, and this has strong relations with algebraic geometry, as it exhibits the special fiber $V$ as the limit of a flat family of complex manifolds that degenerate to $V$.

\section{Extensions AND REFinements OF Milnor's Fibration theOREM}

A natural extension of Milnor's theorem is due to Hamm in [128] (see also [167]) for isolated complete intersection singularities (ICIS). This means a local complete intersection germ

$$
f=\left(f_{1}, \ldots, f_{k}\right): \mathbb{C}^{n+k} \rightarrow \mathbb{C}^{k},
$$

such that $V:=f^{-1}(0)$, has an isolated singularity at the origin. One has a fibration over the regular values of $f$ sufficiently near the origin, where the fiber $F_{t}$ is the intersection of the complex manifold $f^{-1}(t)$ with a small ball around the origin in $\mathbb{C}^{n+k}$. Notice that in this case the set of critical values has in general complex codimension 1 in $\mathbb{C}^{k}$ (see for instance [74, Theorem 1.1]). 
It is proved in [120, Lemma 1.10] (see also [167]) that given an ICIS as above, one can always find good representatives of these singularities, which means that the first $k-1$ equations define an ICIS $\mathcal{W}$ of one dimension more, and the last equation defines an isolated singularity hypersurface germ in $\mathcal{W}$. Therefore, we may see this as a special case of the following theorem from [154] (see [65] for the equivalence of the two fibrations).

Theorem 5.1 (Lê Dũng Tráng). Let $X$ be an analytic subset of an open neighborhood $U$ of the origin $\underline{0}$ in $\mathbb{C}^{n}$. Given $f:(X, \underline{0}) \rightarrow(\mathbb{C}, 0)$ holomorphic with a critical point at $\underline{0} \in X$ (in the stratified sense [116]), let $V:=f^{-1}(0)$, let $\mathbb{B}_{\varepsilon}$ be a closed ball of sufficiently small radius $\varepsilon$ around $\underline{0} \in \mathbb{C}^{n}$, and let $\mathbb{S}_{\varepsilon}$ be its boundary. Then the following hold.

(1) Let $L_{X}=X \cap \mathbb{S}_{\varepsilon}$ be the link of $X$, and let $L_{V}=f^{-1}(0) \cap \mathbb{S}_{\varepsilon}$ be the link of $V$ in $X$. One has a fiber bundle,

$$
\varphi=\frac{f}{|f|}: L_{X} \backslash L_{V} \longrightarrow \mathbb{S}^{1} .
$$

(2) Now choose $\varepsilon \gg \delta>0$ sufficiently small and consider the Milnor tube

$$
N(\varepsilon, \delta)=X \cap \mathbb{B}_{\varepsilon} \cap f^{-1}\left(\partial \mathbb{D}_{\delta}\right)
$$

where $\mathbb{D}_{\delta} \subset \mathbb{C}$ is the disc of radius $\delta$ around $0 \in \mathbb{C}$. Then

$$
f: N(\varepsilon, \delta) \longrightarrow \partial \mathbb{D}_{\delta}
$$

is a fiber bundle, $C^{\infty}$-isomorphic to the previous bundle.

Notice that the fibers in (5.2) are subsets of the link $L_{X}:=X \cap \mathbb{S}_{\varepsilon}$, while the fibers in (5.3) are contained in the interior of $X \cap \mathbb{B}_{\varepsilon}$, in analogy with the classical Milnor fibrations Theorems 4.1 and 4.2. These statements can be refined by giving a fibration on the whole ball $\mathbb{B}_{\varepsilon}$ minus the variety $V:=f^{-1}(0)$ which has the two fibrations in Theorem [5.1 as subfibrations. For this we need the following.

Theorem 5.4 (The Canonical Pencil). For each $\theta \in[0, \pi)$, let $\mathcal{L}_{\theta}$ be the line through 0 in $\mathbb{R}^{2}$ with an angle $\theta$ with respect to the positive orientation of the $x$ axis. Set $V=f^{-1}(0)$ and $X_{\theta}=f^{-1}\left(\mathcal{L}_{\theta}\right)$. Then the following hold.

(i) The $X_{\theta}$ are all homeomorphic real analytic hypersurfaces of $X$ with singular set $\operatorname{Sing}(V) \cup\left(X_{\theta} \cap \operatorname{Sing}(X)\right)$. Their union is the whole space $X$, and they all meet at $V$, which splits each $X_{\theta}$ in two homeomorphic halves.

(ii) If $\left\{S_{\alpha}\right\}$ is a Whitney stratification of $X$ adapted to $V$, then the intersection of the strata with each $X_{\theta}$ determines a Whitney stratification of $X_{\theta}$, and for each stratum $S_{\alpha}$ and each $X_{\theta}$, the intersection $S_{\alpha} \cap X_{\theta}$ meets transversally with every sphere in $\mathbb{B}_{\varepsilon}$ centered at $\underline{0}$.

(iii) There is a uniform conical structure for all $X_{\theta}$, i.e., there is a homeomorphism

$$
h:\left(X \cap \mathbb{B}_{\varepsilon}, V \cap \mathbb{B}_{\varepsilon}\right) \rightarrow\left(\operatorname{Cone}\left(L_{X}\right), \text { Cone }\left(L_{f}\right)\right),
$$

which, when restricted to each $X_{\theta}$, defines a homeomorphism

$$
\left(X_{\theta} \cap \mathbb{B}_{\varepsilon}\right) \cong \operatorname{Cone}\left(X_{\theta} \cap \mathbb{S}_{\varepsilon}\right) \text {. }
$$

The next theorem implies that the fibrations over the circle in Milnor's theorem are actually liftings of fibrations over $\mathbb{R P}^{1}$. 
Theorem 5.5 (Fibration theorem). One has a commutative diagram of fiber bundles

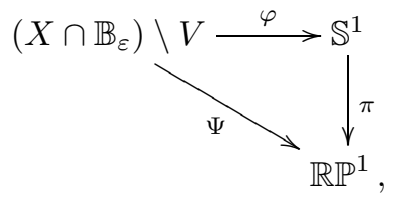

where $\Psi(x)=(\operatorname{Re}(f(x)): \operatorname{Im}(f(x)))$ with fiber $\left(X_{\theta} \cap \mathbb{B}_{\varepsilon}\right) \backslash V, \pi$ is the natural twofold covering, and $\varphi(x)=\frac{f(x)}{|f(x)|}$. The restriction of $\varphi$ to the link $L_{X} \backslash L_{f}$ is the usual Milnor fibration (5.2), while the restriction to the Milnor tube $f^{-1}\left(\partial \mathbb{D}_{\eta}\right) \cap \mathbb{B}_{\varepsilon}$ is the fibration (5.2) (up to multiplication by a constant), and these two fibrations are equivalent.

The proof of Theorem 5.5 follows the same line as in the case where $X$ is nonsingular. The key point is constructing an appropriate integrable vector field in the vein of Lemma 4.3 above. When the ambient space $X$ is singular, we must consider stratified vector fields and use either Mather's controlled vector fields 181 or Verdier's rugose vector fields [274], which are all continuous and integrable. The proof in 65] of Theorem 5.5 also shows the following.

Corollary 5.6. Let $f:(X, \underline{0}) \rightarrow(\mathbb{C}, 0)$ be as above, a holomorphic map with a critical point at $\underline{0} \in X$, and consider its Milnor fibration

$$
\varphi=\frac{f}{|f|}: L_{X} \backslash L_{f} \longrightarrow \mathbb{S}^{1}
$$

If the germ $(X, \underline{0})$ is irreducible, then every pair of fibers of $\varphi$ over antipodal points of $\mathbb{S}^{1}$ are glued together along the link $L_{f}$ producing the link of a real analytic hypersurface $X_{\theta}$, which is homeomorphic to the link of $\{\operatorname{Re} f=0\}$. Moreover, if both $X$ and $f$ have an isolated singularity at $\underline{0}$, then this homeomorphism is in fact a diffeomorphism, and the link of each $X_{\theta}$ is diffeomorphic to the double of the Milnor fiber of $f$ regarded as a smooth manifold with boundary $L_{f}$.

\section{The TOpology of The Fibers: Milnor And LÊ NUMBers}

This section surveys known results about the topology of the Milnor fibers. We refer to Massey's expository articles [178, 179] and lecture notes [177] for more complete accounts of the subject.

We start with an example.

Example 6.1. Consider the homogeneous map $f: \mathbb{C}^{2} \rightarrow \mathbb{C}$ defined by $(x, y) \mapsto x y$; this has a unique critical point at $x=0=y$. Its zero locus $V(f)$ consists of the two axes $\{x=0\} \cup\{y=0\}$ with the origin as an isolated singularity. So its link $L_{f}:=V(f) \cap \mathbb{S}^{3}$ is the Hopf link. By [193, Lemma 9.4], the Milnor fiber $F_{f}$ is diffeomorphic to the whole fiber $f^{-1}(1)$, which consists of the points where $x \neq 0$ and $y=1 / x$. Hence $F_{f}$ is diffeomorphic to a copy of $\mathbb{C}^{*}$; in fact it is an open cylinder $\mathbb{S}^{1} \times \mathbb{R}$, and it can be regarded as being the tangent bundle of the circle. In particular $F_{f}$ has the homotopy type of $\mathbb{S}^{1}$.

We extend these considerations to higher dimensions in two different ways. First notice that we can make the change of coordinates $(x, y) \mapsto\left(z_{1}, z_{2}\right)$ with 
$z_{1}=(x+i y)$ and $z_{2}=(x-i y)$. In these new coordinates the above map becomes $z_{1}^{2}+z_{2}^{2}$, and we may consider, more generally, the homogeneous polynomial

$$
f\left(z_{0}, \ldots, z_{n}\right)=z_{0}^{2}+\cdots+z_{n}^{2} .
$$

The link $L_{f}$ consists of the points where one has

$$
\operatorname{Re}\left(z_{0}^{2}+\cdots+z_{n}^{2}\right)=0, \quad \operatorname{Im}\left(z_{0}^{2}+\cdots+z_{n}^{2}\right)=0, \quad \text { and }\left|z_{0}\right|^{2}+\cdots+\left|z_{n}\right|^{2}=1 .
$$

Hence $L_{f}$ is diffeomorphic to the unit sphere bundle of the $n$-sphere $\mathbb{S}^{n}$. By Milnor's Lemma 9.4 in [193], the Milnor fiber $F_{f}$ is diffeomorphic to the set of points where $z_{0}^{2}+\cdots+z_{n}^{2}=1$, i.e.,

$$
\operatorname{Re}\left(z_{0}^{2}+\cdots+z_{n}^{2}\right)=1 \text { and } \operatorname{Im}\left(z_{0}^{2}+\cdots+z_{n}^{2}\right)=0 .
$$

This describes the tangent bundle of the $n$-sphere $\mathbb{S}^{n}$, and $F_{f}$ actually is the corresponding open unit disc bundle. In particular, $F_{f}$ has the sphere $\mathbb{S}^{n}$ as a deformation retract and therefore $F_{f}$ has nontrivial homology only in dimensions 0 and $n$; in these dimensions its integral homology is isomorphic to $\mathbb{Z}$.

Starting again with the initial example, consider now the map $f: \mathbb{C}^{3} \rightarrow \mathbb{C}$ defined by $(x, y, z) \mapsto x y z$. Its zero set $V(f)$ consists of the coordinate planes $\{x=0\} \cup\{y=0\} \cup\{z=0\}$, with the three axes as a singular set. The Milnor fiber $F_{f}$ is diffeomorphic to $\{x y z=1\}$, i.e., $x \neq 0, y \neq 0$, and $z=1 / x y$. Therefore $F_{f}$ is diffeomorphic to $\mathbb{C}^{*} \times \mathbb{C}^{*}$ and it has the torus $\mathbb{S}^{1} \times \mathbb{S}^{1}$ as a deformation retract. So $F_{f}$ now has nontrivial homology in dimensions 0,1 , and 2 .

We know from 193, that the Milnor fiber $F_{f}$ of an arbitrary holomorphic mapgerm $\left(\mathbb{C}^{n+1}, \underline{0}\right) \rightarrow(\mathbb{C}, 0)$ has the homotopy type of a finite $\mathrm{CW}$-complex of middledimension $n$. This follows too from [12] since $F_{f}$ is a Stein manifold and, perhaps moving the origin $\underline{0}$ slightly if necessary, the square of the function distance to $\underline{0}$ is a strictly plurisubharmonic Morse function on $F_{f}$, so one has severe restrictions on the possible Morse indices.

If we further assume that $f$ has an isolated critical point at $\underline{0}$, then Milnor used Morse theory to show [193, Lemma 6.4] that $F_{f}$ is $(n-1)$-connected. Lefschetz duality, together with the above observations about the homology of $F$, implies that in this setting the fiber $F_{f}$ has the homotopy type of a bouquet of $n$-spheres. The number $\mu=\mu(f)$ of such spheres is now called the Milnor number of $f$. This statement can be made stronger in at least two ways.

On the one hand, we know from [155] that there exists in $F_{f}$ a polyhedron $P$ of middle dimension, with the homotopy type of a bouquet of spheres of dimension $n$, which is a deformation retract of $F_{f}$, and there is a continuous map $F_{f} \rightarrow V$ that carries $P$ into $\underline{0}$ and is a homeomorphism in the complement of $P$. So $F_{f}$ can be thought of as being also a topological resolution of the singularity. This is implicit in 232 for the Pham-Brieskorn polynomials; the polyhedron $P$ is the so-called join of Pham. The theorem in general is in [155] with a sketch of its proof. A complete proof is given in [161].

On the other hand, as proved in 157, 193, the fiber $F_{f}$ is in fact diffeomorphic to a $2 n$-ball with $\mu n$-handles attached. To explain this, we first recall S. Smale's classical process of attaching handles. To attach a $p$-handle to an $m$-manifold $M$, we assume one has a smooth embedding $\iota$ of $S^{p-1} \times D^{m-p}$ into the boundary $\partial M$. Set $H^{p}=D^{p} \times D^{m-p}$ and define a manifold $M \cup_{f} H^{P}$ by taking the disjoint union of $M$ and $H^{p}$ and by identifying $S^{p-1} \times D^{m-p}$ with its image by $\iota$. We think of 
$M \cup_{f} H^{P}$ as being obtained from $M$ by attaching a $p$-handle; the integer $p$ is the index of the handle.

Milnor noticed in 193. that, in high dimensions, Smale's h-cobordism theorem and the fact that the fiber $F_{f}$ has the homotopy type of a bouquet of $n$-spheres actually imply that $F_{t}$ is diffeomorphic to a $2 n$-ball with $\mu n$-handles attached. This claim also holds for $n=1$. The only case left open was $n=2$; this was done by Lê and Perron in [157] by a different method, and their proof actually works in all dimensions. This introduces an important technique which in fact is a first step toward the celebrated Lê's carroussel. The idea is to consider an auxiliary function $\ell: \mathbb{C}^{n+1} \rightarrow \mathbb{C}$, which is linear and "sufficiently general" with respect to $f$. The two maps together determine a map-germ

$$
\varphi=(f, \ell):\left(\mathbb{C}^{n+1}, \underline{0}\right) \rightarrow\left(\mathbb{C}^{2}, 0\right),
$$

and the Milnor fiber of $f$ corresponds to the inverse image of an appropriate line $\ell=c$. This allows us to reconstruct $F_{t}$ by looking at the slices determined by the level hyperplanes of $\ell$ (see Theorem 6.2). This brings us to the remarkable theory of "polar varieties" developed by Bernard Teissier and Lê Dũng Tráng in the 1970s. We define first the relative polar curve of $f$ with respect to a linear form, $\Gamma_{f, \ell}^{1}$ (see for instance [149, 152, 153, 263, 265]).

Given $f$ and $\ell$ as above, as a set the curve $\Gamma_{f, \ell}^{1}$ is the union of those components in the critical set of $(f, \ell)$ which are not in $\Sigma f$, the critical points of $f$. In other words, assume we have coordinates $\left(z_{0}, \ldots, z_{n}\right)$ so that the linear function $\ell=z_{0}$ is sufficiently general. Then the critical locus of $(f, \ell)$ is $V\left(\partial f / \partial z_{1}, \ldots, \partial f / \partial z_{n}\right)$, the set of points where $\partial f / \partial z_{i}=0$ for all $i=1, \ldots, n$. Now write the cycle represented by $V\left(\partial f / \partial z_{1}, \ldots, \partial f / \partial z_{n}\right)$ as a formal sum over the irreducible components,

$$
\left[V\left(\frac{\partial f}{\partial z_{1}}, \ldots, \frac{\partial f}{\partial z_{n}}\right)\right]=\sum n_{i}\left[V_{i}\right]
$$

Then $\Gamma_{f, \ell}^{1}$, as a cycle, is defined by

$$
\Gamma_{f, \ell}^{1}=\sum_{V_{i} \nsubseteq \Sigma f} n_{i}\left[V_{i}\right] .
$$

Now, given $f$, we need to choose the linear form $\ell$ to be "general enough". For this, let us equip $f$ with a good stratification at the singular point $\underline{0}$ in the sense of [129]. This means we have an analytic stratification of a neighborhood $U$ of $\underline{0}$, such that $V(f)$ is a union of strata, the regular set of $V(f)$ is a stratum, and one has Thom's $a_{f}$-condition with respect to $U \backslash V(f)$. We further need $\ell$ to define a prepolar slice for $f$ at $\underline{0}$ with respect to the good stratification $\left\{S_{\alpha}\right\}$. This means that the hyperplane $H=\ell^{-1}(0)$ meets transversely all the strata in $U$, except perhaps the stratum $\underline{0}$ itself.

If $H$ is a prepolar slice for $f$ at $\underline{0}$ defined by a linear form $\ell$, one has that the intersection number $\left(\Gamma_{f, \ell}^{1} \cdot V(f)\right)$ is finite and well-defined. We may now state Lê's attaching theorem.

Theorem 6.2 (Lê's attaching theorem). Given a map-germ $f$ as above, with a possibly nonisolated critical point at $\underline{0}$, consider its local Milnor fiber $F_{f}$ at $\underline{0}$. If $H$ is a prepolar slice for $f$ at $\underline{0}$, then $F_{f}$ is obtained from the Milnor fiber of the slice $V(f) \cap H$ by attaching a certain number of $n$-handles. The number of such handles is the intersection number $\left(\Gamma_{f, \ell}^{1} \cdot V(f)\right)$. 
This leads naturally to the definition of the Lê numbers, introduced by Massey in 175, 177. The idea is similar to the above discussion. A linear functional $\mathbb{C}^{n+1} \rightarrow \mathbb{C}^{r}$ gives rise to a polar variety relative to $f$, determined by the points of nontransversality of the fibers of $\ell$ and $f$. Massey showed that this gives rise to certain local analytic cycles, that he called the Lê cycles, that depend on the choice of the linear functional $\ell$, but they are all equivalent when the form is general enough. These cycles encode deep topological properties of the Milnor fibration. We denote these by $\Lambda_{f, \ell}^{k}(\underline{0})$. Up to equivalence, these are independent of the choice of the prepolar slice.

Each of these analytic cycles has a certain local multiplicity: these are the (generic) Lê numbers $\lambda_{f, \ell}^{k}(\underline{0})$. If the singularity is isolated, then there is only one generic Lê number, and it coincides with the Milnor number. Massey's theorem (see [179, Theorem 3.1] and also 175-177]) tells us how to build up the Milnor fiber by successively attaching handles of various dimensions. This is the second statement in Theorem 6.3 below, and it essentially summarizes the above discussion.

Theorem 6.3. Let $f:\left(\mathbb{C}^{n+1}, \underline{0}\right) \rightarrow(\mathbb{C}, 0)$ be a holomorphic map-germ, and let $F_{f}$ be its Milnor fiber. Then $F_{f}$ is a parallelizable complex Stein manifold that can be regarded as the interior of the compact manifold obtained by attaching to it its boundary; $F_{f}$ has the homotopy type of a $C W$-complex of middle dimension and the following.

(1) If the defining function $f$ has an isolated critical point, then:

- The boundary of $F_{f}$ is isotopic to the link $L_{f}$.

- $F_{f}$ has the homotopy type of a bouquet $\bigvee \mathbb{S}^{n}$ of spheres of middle dimension, and it actually is diffeomorphic to a closed ball $B^{2 n}$ to which we attach $\mu$ n-handles. The number $\mu$ is called the Milnor number of $f$.

- The number $\mu$ can be computed as the intersection number

$$
\mu=\operatorname{dim}_{\mathbb{C}} \frac{\mathcal{O}_{n+1, \underline{0}}}{\operatorname{Jac} \mathrm{f}},
$$

where $\mathcal{O}_{n+1,0}$ is the local ring of germs of holomorphic functions on $\mathbb{C}^{n+1}$ at $\underline{0}$ and Jac $\mathrm{f}$ is the Jacobian ideal generated by the derivatives $\left(\partial f / \partial z_{0}, \ldots, \partial f / \partial z_{n}\right)$.

- There is in $F_{f}$ a polyhedron $P$ of middle dimension, which is a deformation retract of $F_{f}$, and there is a continuous map $F_{f} \rightarrow V$ that carries $P$ into $\underline{0}$ and is a homeomorphism in the complement of $P$.

(2) If the defining function $f$ has a nonisolated critical point:

- If the complex dimension $s$ of its critical set is $s \leq n-2$, then $F_{f}$ is obtained up to diffeomorphism from a $2 n$-ball by successively attaching $\lambda_{f, \ell}^{n-k}(\underline{0}) k$-handles, where $n-s \leq k \leq n$ and $\lambda_{f, \ell}^{n-k}(\underline{0})$ is the $(n-k)$-th Lê number.

- If the complex dimension of its critical set is $s=n-1$, then $F_{f}$ is obtained up to diffeomorphism, from a real $2 n$-manifold with the homotopy type of a bouquet of $\lambda_{f, \ell}^{n-1}(\underline{0})$ circles, by successively attaching $\lambda_{f, \ell}^{n-k}(\underline{0}) k$-handles, where $2 \leq k \leq n$.

The literature about this topic is vast and includes important bouquet theorems by D. Siersma 255] and M. Tibăr 268, for functions defined on singular spaces; both of these theorems are reminiscent of Lê's attaching theorem, Theorem 6.2. 
Remark 6.4 (Vanishing cycles, monodromy and the Milnor lattice). The fibration theorem, Theorem 4.2 tells us that the Milnor fibers can be regarded as a oneparameter flat family $\left\{F_{t}\right\}$ of complex manifolds that degenerate to the special fiber $F_{0}=V:=f^{-1}(0)$. Since $V$ is a topological cone, this means that all the homology groups of $F$ vanish in the limit. In particular, if the critical point of $f$ at $\underline{0}$ is isolated, then Theorem 6.3 says that the only interesting homology group of the Milnor fiber $F$ is in dimension $n$ and it is generated by $\mu(f)$ cycles of dimension $n$, which are called the vanishing cycles. This group,

$$
\mathcal{L}(f):=H_{n}(F, \mathbb{Z}) \cong \mathbb{Z}^{\mu(f)},
$$

is naturally equipped with a $(-1)^{n}$-symmetric bilinear form $\langle$,$\rangle coming from the$ intersection of cycles. This group $\mathcal{L}(f)$ together with this additional structure is called the Milnor lattice of the singularity. The literature about it is vast; we refer to Dimca's book [71] for an account of this subject.

The $E_{8}$ lattice is an example of a unimodular lattice which is rather famous in singularity theory as well as in low dimensional topology. This is the Milnor lattice of the singularity $z^{2}+z_{2}^{3}+z_{3}^{5}=0$ in $\mathbb{C}^{3}$, whose link is the celebrated Poincaré homology 3-sphere.

A cornerstone in the study of Milnor lattices is the monodromy of the fibration. For this, it is useful to consider distinguished bases of the vanishing cycles. We refer for this to $[71,83,84,103,243,253$. Another viewpoint for studying the monodromy is via its zeta-function; see for instance [1,2, 125, 218, 272] for some classical material, or [19, 49, 60 and their bibliographies for more recent work on the subject.

\section{On the Milnor nUmber}

Given a map-germ $\left(\mathbb{C}^{n+1}, \underline{0}\right) \stackrel{f}{\rightarrow}(\mathbb{C}, 0)$ with an isolated critical point at $\underline{0}$, we know from the previous section that $F$ has the homotopy type of a bouquet $\bigvee \mathbb{S}^{n}$ of spheres of middle dimension. Therefore all its homology groups vanish except in dimension 0 where it is $\mathbb{Z}$, and in dimension $n$ where it is free abelian of rank $b_{n}$. The Milnor number $\mu:=\mu(f)$ is defined as the $n$th Betti number $b_{n}(F)$.

Theorem 8.2 in Milnor's book 193 says that $\mu(f)$ equals the multiplicity of the map-germ $f$ at $\underline{0}$, which equals the local Poincaré-Hopf index of its gradient vector field $\nabla f(z):=\left(\partial f / \partial z_{0}(z), \ldots, \partial f / \partial z_{n}(z)\right)$. Since the vector field $\nabla f$ is holomorphic, standard arguments in algebraic geometry say this index is the intersection number in Theorem 6.3.

$$
\mu=\operatorname{dim}_{\mathbb{C}} \frac{\mathcal{O}_{n+1, \underline{0}}}{\operatorname{Jac} \mathrm{f}} .
$$

It follows that the Euler characteristic of the fiber $F$ is $\chi(F)=1+(-1)^{n} \mu$. If we think of $F$ as being a compact manifold with boundary the link $L_{f} \cong \partial F$, then the theorem of Poincaré-Hopf for manifolds with boundary says that $\chi(F)$ is the total Poincaré-Hopf index of a vector field in $F$ that points outward at each point of the boundary.

We know from [128, that one has a Milnor fibration for ICIS germs:

$$
f:=\left(f_{1}, \ldots, f_{k}\right):\left(\mathbb{C}^{n+k}, \underline{0}\right) \rightarrow(\mathbb{C}, 0) .
$$

Hamm proved that in this setting, the Milnor fiber also has the homotopy type of a bouquet of spheres of middle dimension. So ICIS germs have also a well-defined Milnor number, defined as the rank of the middle homology of the Milnor fiber. It 
is thus natural to search for an algebraic expression for the Milnor number of ICIS germs in the vein of (17.1). This is known as the Lê--Greuel formula for the Milnor number:

Theorem 7.2. If $f_{1}, \ldots, f_{k}$ and $g$ are holomorphic map-germs $\left(\mathbb{C}^{n+k+1}, \underline{0}\right) \rightarrow$ $(\mathbb{C}, 0)$ such that $f=\left(f_{1}, \ldots, f_{k}\right)$ and $(f, g)$ define ICIS germs, then their Milnor numbers are related by

$$
\mu(f)+\mu(f, g)=\operatorname{dim}_{\mathbb{C}} \frac{\mathcal{O}_{n+k, \underline{0}}}{\left(f, \operatorname{Jac}_{k+1}(f, g)\right)},
$$

where $\operatorname{Jac}_{k+1}(f, g)$ denotes the ideal generated by the determinants of all $(k+1)$ minors of the corresponding Jacobian matrix.

This formula was proved independently by Lê [150] and G.-M. Greuel [118]. At about the same time Teissier proved [263, Proposition II.1.2], a "formule de restriction", which is the same theorem in the case where one of the two functions is linear; this is known as Teissier's lemma. We also have the celebrated Teissier sequence of numbers of an ICIS, which are the Milnor numbers of the corresponding complete intersection germs one gets by taking linear slices of various dimensions. This is briefly discussed in the following section. We refer to [56, 63, 81 for other recent viewpoints on the Lê- - Greuel formula for the Milnor number.

There are two questions that arise naturally.

Question 7.4. What is (or what ought to be) the Milnor number of a nonisolated hypersurface or complete intersection singularity?

Question 7.5. What is (or what ought to be) the Milnor number of an isolated complex analytic singularity $(V, P)$ which may not be an ICIS?

There is a vast literature about both of these questions, with different viewpoints. Concerning the first of these, there are two particularly interesting viewpoints. One, due to D. Massey, is via the Lê numbers of $V$, explained in section 6. These describe the topology of the local Milnor fiber (cf. Theorem 6.3), and when the singularity is isolated, there is only one Lê number and it coincides with the Milnor number.

Another viewpoint is that of Parusiński in 227. He considers a compact complex manifold $M$ and a codimension 1 subvariety $V$ defined by a holomorphic section of some line bundle $L$ over $M$. There is a Milnor number $\mu(S ; V)$ associated to each connected component of its singular set. This has important properties; some of these are listed here.

(i) It coincides with the usual Milnor number when the singularity is isolated.

(ii) Its total index in $V$ equals the 0-degree Fulton-Johnson class (cf. section 9).

(iii) If we can approximate $V$ by a family of manifolds $\left\{V_{t}\right\}$ defined by sections of $L$ which are transversal to the zero section, then $\mu(S ; V)$ measures the change in the Euler characteristic as the $\left\{V_{t}\right\}$ degenerate into $V$.

(iv) For complex polynomials $f: \mathbb{C}^{m} \rightarrow \mathbb{C}$, it measures the change in the Euler characteristic of the fibers at infinity $[20$.

Concerning Question 7.5, for a reduced complex curve $(X, P)$, Buchweitz and Greuel in 52] define the Milnor number by

$$
\mu_{\mathrm{BG}}:=\operatorname{dim} \frac{\omega_{X}}{d \mathcal{O}_{X}},
$$


where $\omega_{X}$ is Grothendieck's dualizing module (cf. [195]). When the germ $(X, P)$ is smoothable, it equals the first Betti number of the smoothing, so for ICIS it coincides with the usual Milnor number. We recall what smoothable means.

Definition 7.6. A normal isolated singularity germ $(X, P)$ of pure dimension $n$ is smoothable if there exists an $(n+1)$-dimensional complex analytic space $\mathcal{W}$ with an isolated normal singularity that we also denote $P$, and a flat morphism

$$
\mathcal{G}:(\mathcal{W}, P) \longrightarrow(\mathbb{C}, 0),
$$

such that $\mathcal{G}^{-1}(0)$ is $X$ and $\mathcal{G}^{-1}(t)$ is nonsingular for all $t \neq 0$ with $|t|$ sufficiently small.

The fibers of a smoothing give a similar picture to that of the Milnor fibration of a hypersurface singularity, the difference being that $\mathcal{W}$ may now be singular.

The above definition in [52] of the Milnor number was generalized in 50] to isolated not necessarily reduced curve singularities, and in [117] to isolated singularities of arbitrary dimension.

There is in 88 an invariant $\nu$ that can be regarded as an "alternative Milnor number". This is defined in all dimensions using indices of 1 -forms (cf. section 8 below). One has in [88] a Lê-Greuel type formula for $\nu$, proved using Greuel's work [118. For curves one gets

$$
\nu:=\operatorname{dim} \Omega_{X} / d \mathcal{O}_{X}
$$

where $\Omega_{X}$ is the canonical module of holomorphic 1-forms, so $\nu$ coincides with the Milnor number in 52 , when $X$ is an ICIS.

In dimension 2, Greuel and Steenbrink proved in 121 that if an isolated surface singularity $(V, \underline{0})$ is normal and Gorenstein, then its first Betti number $b_{1}(V, \underline{0})$ vanishes and $b_{2}(V, \underline{0})$ is independent of the choice of the smoothing. Hence every such germ has a well-defined Milnor number $\mu_{\mathrm{GS}}(V, \underline{0}):=b_{2}(V, \underline{0})$. One has the Laufer-Steenbrink formula for the Milnor number, proved in [146] for hypersurfaces (the same proof works for ICIS), and by Steenbrink [257] in general.

Theorem 7.7. Assume that $(V, 0)$ is a smoothable Gorenstein normal surface singularity. Then

$$
\mu_{\mathrm{GS}}+1=\chi(\widetilde{V})+K^{2}+12 \rho_{g}(V),
$$

where $\chi(\widetilde{V})$ is the usual Euler characteristic of a good resolution, $K^{2}$ is the selfintersection number of the canonical class of $\widetilde{V}$, and $\rho_{g}:=\operatorname{dim} H^{1}(\widetilde{V}, \mathcal{O})$ is the geometric genus.

For surfaces, the Milnor number $\nu$ defined in 88 seems to coincide with $\mu_{\mathrm{GS}}$.

\section{INDiCES OF VECTOR FIELDS AND THE MILnOR NUMBER}

The Poincaré-Hopf local index of a vector field on a smooth manifold is a fundamental invariant that has given rise to a vast literature. In the case of vector fields on singular varieties, there are several extensions of this concept, each having its own properties and characteristics (see [41] for a thorough account of the subject).

In the case of manifolds, up to sign issues, it is essentially the same to consider indices of 1 -forms or vector fields. In the case of singular varieties, this is no longer the case, though the two theories are parallel and have many similarities. The case of 1-forms has been developed mostly by W. Ebeling and S. Gusein-Zade (see for instance [85 88, or [41, Chapter 9]). Here we focus on vector fields. 


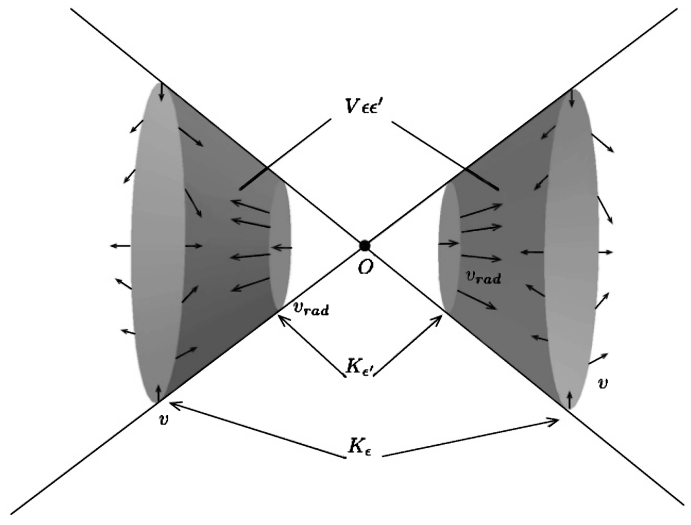

(a)

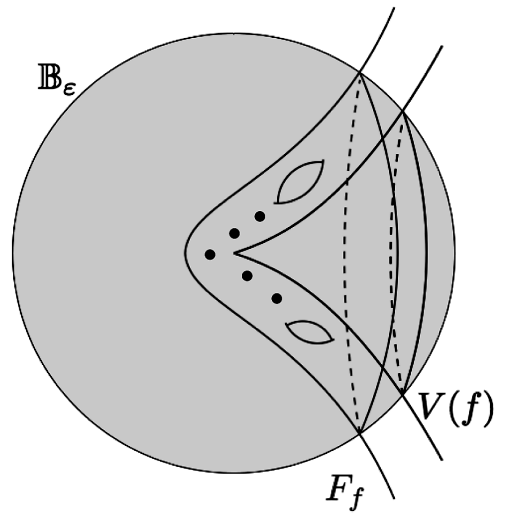

(b)

FIgURE 5. The (a) radial and (b) GSV indices.

Let $(V, \underline{0})$ be an analytic variety of dimension $n$ (say, irreducible and reduced) for simplicity, with a unique singular point in an open set $U$ in some $\mathbb{C}^{m}$, and let $v$ be a vector field tangent to $V$, singular only at $\underline{0}$, and restricting a continuous vector field in $U$. Then one has several possible notions of its local index:

(i) the radial index;

(ii) the GSV index;

(iii) the virtual index;

(iv) the homological index;

(v) the local Euler obstruction (see section 8.1).

Let us say a few words about these. The Euler obstruction is discussed on its own in section 8.1 .

(i) The radial index. This is reminiscent of the Schwartz index in 244, defined only for radial vector fields. The radial index was introduced in [141] and then, independently, in 44,85; see Figure 5(a). This is defined for continuous stratified vector fields on arbitrary compact (real or complex) varieties equipped with a Whitney stratification (and even more generally, see [141]), and it measures the lack of radiality of the vector field:

$$
\operatorname{Ind}_{\text {rad }}(v ; \underline{0}, V)=1+d\left(v, v_{\text {rad }}\right),
$$

where $d\left(v, v_{\text {rad }}\right)$ is the total Poincaré-Hopf index of a vector field in a cylinder in $V$ bounded by two copies of the link, and which is radial in the "smaller" link and coincides with $v$ in the "larger" link.

The extension of this index for vector fields on varieties with nonisolated singularities is straightforward (see [4,41]).

(ii) The GSV index. The GSV index was the first "index of vector fields" in the literature defined in general, since the Schwartz index and the local Euler obstruction were defined only for radial vector fields; see Figure 5(b). To define the GSV index, we need $V$ to be a complete intersection. For simplicity we assume $(V, \underline{0})$ is a hypersurface defined by some map $f$ in an open neighborhood $U$ of $\underline{0}$ in $\mathbb{C}^{n+1}$. Given a continuous vector field $v$ tangent to $V$ and singular only at $\underline{0}$, we notice that $(\bar{\nabla} f, v)$ determine a continuous map from the link $L_{f}$ to the Stiefel 
manifold of complex orthonormal 2-frames in $\mathbb{C}^{n+1}$. Such maps have a degree, and this is the GSV index of $v, \operatorname{Ind}_{\mathrm{GSV}}(v ; \underline{0}, V)$; see [41,111 for details.

The GSV index can be interpreted as follows: We can always assume that $v$ is the restriction of a continuous vector field $\tilde{v}$ on $U$, which is tangent to the Milnor fibers $F_{t}$ for all $t$ sufficiently near to $0 \in \mathbb{C}$, and the restriction $v_{t}$ of $\tilde{v}$ to each $F_{t}$ has finitely many singularities. As $t$ tends to 0 , the fibers $F_{t}$ degenerate to the special fiber $V$ and the vector fields $v_{t}$ degenerate to $v$. If $V$ is irreducible, then $\operatorname{Ind}_{\mathrm{GSV}}(v ; \underline{0}, V)$ equals the total Poincaré-Hopf index of each $v_{t}$.

One has (see [41]):

Proposition 8.1. Given an ICIS germ $(V, \underline{0})$ and a continuous vector field $v$ on $V$ singular only at $\underline{0}$, the difference of the radial and $G S V$ indices is independent of the vector field and equals the Milnor number up to sign:

$$
\mu(f)=(-1)^{n}\left(\operatorname{Ind}_{\mathrm{GSV}}(v ; \underline{0}, V)-\operatorname{Ind}_{\mathrm{rad}}(v ; \underline{0}, V)\right) .
$$

(iii) The virtual index. The virtual index was defined in 162 for $v$ holomorphic and extended to continuous vector fields in 251. The idea is very simple. Recall first that classical Chern-Weil theory tells us how to construct the Chern classes of complex manifolds out of a connection. Recall too that the Gauss-Bonnet formula allows us to identify the Euler characteristic $\chi(M)$ of a compact complex $m$-manifold $M$ with $c_{m}(M)[M]$, the top Chern class of $M$ evaluated on the orientation cycle. When we have a vector field with singularities on $M$, we can follow the classical Baum-Bott theory and construct a special connection which, around each connected component of the singular set $S$ of $v$, is $v$-trivial. This yields a curvature form on $M$ which determines a representative of $c_{m}(M)$ that vanishes away from a regular neighborhood of $S$. We thus get an expression for $c_{m}(M)[M]$ which is localized at $S$. When $S$ consists of isolated points, the contribution of $v$ at each singularity is the Poincaré-Hopf local index of $v$. The point is that exactly the same idea goes through for vector fields on a complete intersection $V$ in a compact complex manifold $M$, defined by a regular section of a rank $k$ vector bundle $E$ over $M$. Now we have the virtual tangent bundle of $V$, which by definition is $\tau V:=\left.T M\right|_{V}-\left.E\right|_{V}$. This virtual bundle has well-defined Chern classes, and just as above, a continuous vector field $v$ on $V$ allows us to localize the top Chern class of this virtual bundle at the singular set $S$ of $v$. What we get is the virtual index of $v$ associated to each connected component of $S$. When the singularities of both $V$ and $v$ are isolated, the virtual index coincides with the local GSV index (see [41,162]). One has [41, Theorem 6.2]:

Theorem 8.2. Let $V$ be a global complete intersection in a complex manifold $M$, and let $S$ be a connected component of the singular set of $V$. Let $v$ be a continuous vector field defined in a neighborhood of $S$ in $V$, with no singularities away from $S$. Then the difference between the radial and the GSV indices is independent of $v$. Furthermore, if $V$ has codimension 1 in $M$, then this difference is Parusinski's Milnor number up to sign: $\mu(S ; V)=(-1)^{n}\left(\operatorname{Ind}_{\mathrm{Vir}}(v ; S, V)-\operatorname{Ind}_{\mathrm{rad}}(v ; S, V)\right)$.

(iv) The homological index. Using the fact that when the ambient space is smooth, the Poincaré-Hopf local index can be interpreted as the Euler characteristic of a certain Koszul complex, Gómez-Mont introduced in 112 the notion of a homological index of holomorphic vector fields. Let us explain this invariant. Let $(V, \underline{0}) \subset\left(\mathbb{C}^{m}, \underline{0}\right)$ be a germ of a complex analytic variety of pure dimension $n$, 
which is regular on $V \backslash\{\underline{0}\}$. A vector field $v$ on $(V, \underline{0})$ can always be defined as the restriction to $V$ of a vector field $\widehat{v}$ in the ambient space which is tangent to $V \backslash\{\underline{0}\} ; v$ is holomorphic if $\widehat{v}$ can be chosen to be holomorphic. So we may write $v$ as $v=\left(a_{1}, \ldots, a_{m}\right)$, where the $a_{i}$ are restrictions to $V$ of holomorphic functions on a neighborhood of $\underline{0}$ in $\mathbb{C}^{m}$.

A (germ of) holomorphic $j$-form on $V$ at $\underline{0}$ means the restriction to $V$ of a holomorphic $j$-form on a neighborhood of $\underline{0}$ in $\mathbb{C}^{m}$. We denote by $\Omega_{V, \underline{0}}^{j}$ the space of all such forms (germs); these are the Kähler differential forms on $V$ at $\underline{0}$. So $\Omega_{V, \underline{0}}^{0}$ is the local structure ring $\mathcal{O}_{(V, \underline{0})}$ of holomorphic functions on $V$ at $\underline{0}$ and each $\Omega_{V, \underline{0}}^{j}$ is an $\Omega_{V, \underline{0}}^{0}$-module. Now, given a holomorphic vector field $\widehat{v}$ at $\underline{0} \in \mathbb{C}^{m}$ with an isolated singularity at the origin and a differential form $\omega \in \Omega_{\mathbb{C}^{m}, \underline{0}}^{j}$, we can always contract $\omega$ by $v$ in the usual way, thus getting a differential form $i_{v}(\omega) \in \Omega_{\mathbb{C}^{m}, 0}^{j-1}$. If $v=\left.\widehat{v}\right|_{V}$ is tangent to $V$, then contraction is well-defined at the level of differential forms on $V$ at $\underline{0}$, and one gets a complex $\left(\Omega_{V, \underline{0}}^{\bullet}, v\right)$ :

$$
0 \longrightarrow \Omega_{V, \underline{0}}^{n} \longrightarrow \Omega_{V, \underline{0}}^{n-1} \longrightarrow \cdots \longrightarrow \mathcal{O}_{V, \underline{0}} \longrightarrow 0,
$$

where the arrows are contractions by $v$, and $n$ is the dimension of $V$. We consider the homology groups of this complex:

$$
H_{j}\left(\Omega_{V, \underline{0}}^{\bullet}, v\right)=\operatorname{Ker}\left(\Omega_{V, \underline{0}}^{j} \rightarrow \Omega_{V, \underline{0}}^{j-1}\right) / \operatorname{Im}\left(\Omega_{V, \underline{0}}^{j+1} \rightarrow \Omega_{V, \underline{0}}^{j}\right) .
$$

Since the contraction maps are $\mathcal{O}_{V, \underline{0}}$-module maps, this implies that if $V$ has an isolated singularity at the origin, then the homology groups of this complex are concentrated at $\underline{0}$, and they are finite dimensional because the sheaves of Kähler differentials on $V$ are coherent. Hence it makes sense to define the following.

Definition 8.4. The homological index $\operatorname{Ind}_{\text {hom }}(v, \underline{0} ; V)$ of a holomorphic vector field $v$ on $(V, \underline{0})$ with an isolated singularity at $\underline{0}$ is the Euler characteristic of the above complex,

$$
\operatorname{Ind}_{\text {hom }}(v, \underline{0} ; V)=\sum_{i=0}^{n}(-1)^{i} h_{i}\left(\Omega_{V, \underline{0}}^{\bullet}, v\right),
$$

where $h_{i}\left(\Omega_{V, \underline{0}}^{\bullet}, v\right)$ is the dimension of the corresponding homology group as a vector space over $\mathbb{C}$.

The homological index coincides with the GSV index when the germ of $V$ is a complete intersection, by [36]. Hence in this case its difference with the radial index is the Milnor number. Yet, the homological and the radial index are defined for holomorphic vector fields on arbitrary normal isolated complex singularity germs. It is an exercise to show that their difference does not depend on the choice of vector field, so it is an invariant of the germ $(V, \underline{0})$. This leads us to a question.

Question 8.5. If $(V, \underline{0})$ is a normal isolated complex singularity germ which is not a complete intersection, and $v$ is a holomorphic vector field tangent to $V$ and is nonsingular away from $\underline{0}$, what is the difference between its homological and radial indices?

8.1. The local Euler obstruction. MacPherson defined in [174] the notion of the local Euler obstruction of a complex analytic space at each of its points. This was a key ingredient for constructing Chern classes for singular varieties. 
The idea is that whenever we have a singular variety $V$ in some complex manifold $M$, we can consider its Nash transform $\widetilde{V}$. This is the analytic space obtained by removing from $V$ its singular set, and replacing this by all limits of tangent spaces over the regular part $V_{\text {reg. So }}$. Se may think of it as being a blowup. The space $\widetilde{V}$ itself is again singular in general, but it has the nice property that it has a natural projection $\widetilde{V} \stackrel{\nu}{\rightarrow} V$ which is a biholomorphism over $V_{\text {reg }}$, and $\widetilde{V}$ is naturally equipped with a vector bundle $\widetilde{T}$ called the Nash bundle, which over $\nu^{-1}\left(V_{\text {reg }}\right)$ is isomorphic to the tangent bundle.

Recall that if we equip $V$ with a Whitney stratification (a stratified vector field means the restriction to $V$ of a continuous vector field $v$ in a neighborhood of $V$ in $M)$ such that for each $x \in V$, the vector $v(x)$ is contained in the space tangent at $x$ to the corresponding stratum. We know from [37 that the Whitney $a$-condition implies that every stratified vector field on $V$ has a natural lifting to a section of $\widetilde{T}$.

We may now consider a local index $\operatorname{Eu}_{V}(v, x)$ of vector fields as follows: Given $V$ as above (and equipped with a Whitney stratification), a point $x \in V$ and a stratified vector field $v$ in a neighborhood $U$ of $x$, which is nonsingular away from $x$, we lift it to a nowhere-zero section $\tilde{v}$ of the Nash bundle $\widetilde{T}$ over $\nu^{-1}(U \backslash\{x\})$. Then $\operatorname{Eu}_{V}(v, x)$ is an integer which is the obstruction to extending $\tilde{v}$ as a nowherezero section of $\widetilde{T}$ over $\nu^{-1}(U)$.

A stratified vector field $v$ in a neighborhood of a point $x_{o}$ in $V$ is radial at $x_{o}$ if there is small ball $\mathbb{B}_{\varepsilon}$ in $M$, such that $v(x)$ is pointing outward the ball at each point $x \in \mathbb{S}_{\varepsilon} \cap V$, where $\mathbb{S}_{\varepsilon}:=\partial \mathbb{B}_{\varepsilon}$ is the boundary sphere.

Definition 8.6. The local Euler obstruction $\operatorname{Eu}_{V}(x) \in \mathbb{Z}$ of $V$ at a point $x$ is the local index $\operatorname{Eu}_{V}\left(v_{\mathrm{rad}}, x\right)$ of a stratified vector field which is radial at $x$.

Besides its importance for MacPherson's proof of the Deligne-Grothendieck conjecture that we discuss in section [9, this index has important relations with the Milnor number when $V$ is an ICIS (see [39,75]) and with various important invariants of singular varieties (cf. 40, 86, 87, 115, 124, 158, 252 ). In particular one has Dubson's theorem [75]:

Theorem 8.7. Let $f:\left(\mathbb{C}^{n+1}, \underline{0}\right) \rightarrow(\mathbb{C}, 0)$ be holomorphic with an isolated critical point at $\underline{0}$, and let $H$ be a generic hyperplane in $\mathbb{C}^{n+1}$ passing through $\underline{0}$. Set $V=f^{-1}(0)$. Then $\operatorname{Eu}_{V}(\underline{0})$ is determined by the Milnor number of the hyperplane section $V \cap H$. More precisely,

$$
\operatorname{Eu}_{V}(\underline{0})=1+(-1)^{n} \mu(V \cap H) .
$$

This generalizes to varieties with arbitrary singular locus 39 . If we cut down $V$ by a linear form which is not general enough or by a function with an isolated critical point on $V$ at $\underline{0}$, the above formula gets a correction term: this is the Euler defect in [40].

\section{Milnor Classes}

Milnor classes are another generalization of the classical Milnor number to varieties with arbitrary singularities. These measure the difference between two natural extensions for singular varieties of the classical Chern classes, namely the total Schwartz-MacPherson class $c_{*}^{S M}(X)$ (see [174,244]) and the total Fulton-Johnson class $c_{*}^{F J}(X)$ (see [101]). By definition, the total Milnor class is

$$
\mathcal{M}(X):=(-1)^{\operatorname{dim} X}\left(c^{F J}(X)-c^{S M}(X)\right) .
$$


Given the category of compact algebraic varieties, one has the functor $\mathcal{F}(X)$ that assigns to each such variety $X$ the abelian group of $\mathbb{Z}$-valued constructible functions on $X$. One also has the funtor $H_{*}(X)$ that assigns to $X$ its singular homology with integer coefficients. In 1974 MacPherson [174] settled a conjecture of Deligne and Grothendieck stating that there is a unique natural transformation from the functor $\mathcal{F}$ to homology, associating to the constant function $\mathbb{1}_{X}$ on a nonsingular variety $X$ the Poincaré dual of the total Chern class of the tangent bundle $T X$ of $X$ (cf. [258, p. 168] and Grothendieck's own comments in [123, Note 871, p. 376]). Therefore, for a possibly singular variety $X$, the homology class corresponding to the function $\mathbb{1}_{X}$ is a natural candidate for a notion of Chern class. It was proved in 37] that these classes agree, up to Alexander duality, with certain classes defined earlier by M.-H. Schwartz in analogy with the classical definition of Chern classes via obstruction theory. Hence these are known as Schwartz-MacPherson classes. In particular, the 0-degree class is an integer and it equals the Euler characteristic, essentially by definition. A key ingredient in MacPherson's proof of the DeligneGrothendieck conjecture is the local Euler obstruction introduced in section 7.

On the other hand, whenever we have a subscheme $X$ of a nonsingular variety $M$, its Fulton class [101] is the cap product of the total Chern class of $M$ with the Segre class of $X$ :

$$
c_{F}(X):=c\left(\left.T M\right|_{X}\right) \cap s(X, M) .
$$

When $X$ is an $(n-k)$ local complete intersection in $M$, defined by a regular section of some rank $k$ holomorphic bundle $E$ over $M$, the Fulton class is the total Chern class of the virtual bundle $\tau X:=\left.T M\right|_{X}-\left.E\right|_{X}$, and it coincides with the FultonJohnson class previously defined in [102]. Fulton proved in [101] that $c_{F}(X)$ is independent of $M$.

In the complex analytic context, Milnor classes are elements in the homology group $H_{2 *}(X, \mathbb{Z})$, and in the algebraic context, these can be lifted to elements in the Chow group $A_{*}(X)$.

Just as Chern classes are related to the local index of Poincaré-Hopf, so too, the Schwartz-MacPherson and the Fulton-Johnson classes are related to the radial and virtual indices defined in section 8 (see 41]).

We know from 38, 261 that for local complete intersections the total Milnor class actually has support in the singular set $\operatorname{Sing}(X)$ of $X$, and there is a Milnor class in each dimension, from 0 to that of $\operatorname{Sing}(Y)$. Presumably, this also happens in general for varieties with arbitrary singular locus, but to my knowledge, this has not yet been proved.

It follows that if $X$ is a complete intersection and its singularities are all isolated, then there is only a 0-degree Milnor class, which is an integer, and we know from 251. that this integer is the sum of the local Milnor numbers. This is a consequence of Proposition 8.1, the fact that the 0-degree Schwartz-MacPherson class can be regarded as being the total radial index of a continuous vector field on $X$, and that for complete intersections, the 0-degree Fulton-Johnson class equals the total GSV index of a vector field on $X$. This justifies the name "Milnor classes".

Milnor classes spring from 9], and they are an active field of current research with significant applications to other related areas. There is a large literature on Milnor classes, for instance [10, 11, 25, 38, 41, 42, 53, 55, 58, 182, 183, 217, 230, 245. Milnor classes encode much information about the varieties in question, and this is being studied by various authors from several points of view. 
Most of the literature on Milnor classes is for hypersurfaces, though there are some recent works that throw light on the subject in the case of complete intersections. In [54 the authors study the total Milnor class of complete intersections $Z(s)$ defined by a regular section $s$ of a rank $r$ holomorphic bundle $E$ over a compact manifold $M$. It is noticed that $s$ determines a hypersurface $Z(\tilde{s})$ in the total space of the projectivization $\mathbb{P}\left(E^{\vee}\right)$ of the dual bundle $E^{\vee}$, and one has a formula expressing the total Milnor class of $Z(s)$ in terms of the Milnor classes of the hypersurface $Z(\tilde{s})$. This means that morally, everything known for the Milnor classes of hypersurfaces is also known for complete intersections.

In [55] there is a surprisingly simple formula for the total Milnor class when $X$ is defined by a finite number of hypersurfaces $X_{1}, \ldots, X_{r}$ in a complex manifold $M$, satisfying certain transversality conditions:

$$
\begin{aligned}
\mathcal{M}(X) & =(-1)^{\operatorname{dim} X} c\left(\left(\left.T M\right|_{X}\right)^{\oplus r-1}\right)^{-1} \\
& \cap\left(c^{F J}\left(X_{1}\right) \cdots c^{F J}\left(X_{r}\right)-c^{S M}\left(X_{1}\right) \cdots c^{S M}\left(X_{r}\right)\right) .
\end{aligned}
$$

Notice that for $r=1$ this is just the definition of the class $\mathcal{M}(X)$. There is also [246] where the author gives a general transversality formula that throws light on the theory of Chern classes for complete intersections.

For varieties which are not complete intersections, essentially nothing is known regarding their Milnor classes. In fact, there is even an ambiguity in the definition, since it is not clear if one should consider the Fulton or the Fulton-Johnson class [102, which coincide for complete intersections; perhaps both and get two different interesting concepts. Here is an easy way to state the question (cf. Question 7.5).

Question 9.2. Consider an algebraic normal isolated singularity germ $(V, P)$ of dimension $n \geq 1$ which is not a complete intersection. Consider a projective compactification of it, and resolve its singularities at infinity. We get a compact variety $\bar{V}$ with an isolated singularity. What is the difference between its Fulton and Schwartz-MacPherson classes?

\section{Equisingularity}

The vast field of equisingularity theory emerged after the seminal work of $O$. Zariski [281 284, B. Teissier (see for instance [263, 264,266]), Lê Dũng Tráng (see for instance [151, 156, 160]), and many others, for instance [94, 95, 104 106, 176, 216]. A recent extensive survey with open problems is given in [117.

The term "equisingular" refers to a relation of equivalence which formalizes the intuitive idea of singularities of "the same type" in some sense. In general one would like to find conditions, and perhaps numerical invariants, that grant some type of equisingularity. There are several important notions of equisingularity. The most basic one is as follows.

Definition 10.1. Two germs of reduced complex analytic hypersurfaces $\left(V_{1}, q_{1}\right)$ and $\left(V_{2}, q_{2}\right)$ in $\mathbb{C}^{n+1}$ are topologically equisingular if they have the same embedded topological type, i.e., if there exist representatives of these germs $\left(V_{i}, q_{i}\right) \subset\left(U_{i}, q_{i}\right)$, $i=1,2$, with $U_{i}$ an open set in $\mathbb{C}^{n+1}$, and a homeomorphism of pairs $\left(U_{1}, q_{1}\right) \cong$ $\left(U_{2}, q_{2}\right)$ taking $V_{1}$ into $V_{2}$.

One has the celebrated Question A by Zariski in [285, known as Zariski's multiplicity conjecture: 
Question 10.2. Does topological equisingularity of two hypersurface germs $V_{1}$ and $V_{2}$ at $q_{1}$ and $q_{1}$ imply that these have the same multiplicity, $\nu\left(V_{1}, q_{1}\right)=\nu\left(V_{2}, q_{2}\right)$ ?

Recall that if $f:\left(\mathbb{C}^{n+1}, \underline{0}\right) \rightarrow(\mathbb{C}, 0)$ is a holomorphic map-germ, we can write it in terms of its Taylor series expansion,

$$
f(z)=\sum_{j=1}^{\infty} f^{j}(z), \quad \text { with } f^{j}(z)=\sum_{\alpha_{0}+\cdots+\alpha_{n}=j} a_{\alpha} z^{\alpha},
$$

where each $f^{j}$ is homogeneous of degree $j, \alpha=\left(\alpha_{0}, \ldots, \alpha_{n}\right) \in \mathbb{N}^{n+1}, z^{\alpha}=$ $z_{0}^{\alpha_{1}} \cdots z_{n}^{\alpha_{n}}$ and $a_{\alpha} \in \mathbb{C}$. The order of $f$ at $\underline{0}$ is the smallest degree $j$ such that $f^{j}$ is not identically 0 . If the germ is reduced, this coincides with its multiplicity $\nu(f, \underline{0})$, which by definition is the number of points of intersection, near $\underline{0}$, of $V=f^{-1}(0)$ with a generic complex line in $\mathbb{C}^{n+1}$ passing arbitrarily close to $\underline{0}$ but not through $\underline{0}$.

There is a vast literature about this question, known as Zariski's multiplicity conjecture, with many partial answers. We refer to 93 for a survey on the topic, and to 98 for the answer to a metric version.

Another important notion of equisingularity is Whitney regularity, which has already appeared several times in this work. The existence of Whitney stratifications for every analytic space $X$ was proved by Whitney in [278, Theorem 19.2] for complex varieties, and by Hironaka [132] in a more general setting. Thom [267] and Mather [181] proved that Whitney equisingularity implies local topological triviality; this is essentially a consequence of the first Thom isotopy lemma (cf. [3, 274]).

Definition 10.3. Let $V$ be a reduced complex analytic hypersurface in $\mathbb{C}^{n+1}$, and let $V_{\alpha} \subset V$ be a stratum of some Whitney stratification. We say that $V$ is topologically trivial along $V_{\alpha}$ at a point $x \in V_{\alpha}$ if there is a neighborhood $W$ of $x$ in $\mathbb{C}^{n+1}$, homeomorphic to $\Delta \times U_{\alpha}$, where $U_{\alpha}$ is a neighborhood of $x$ in $V_{\alpha}$ and $\Delta$ is a small closed disk through $x$ of complex dimension $(n+1)-\operatorname{dim}_{\mathbb{C}} V_{\alpha}$, transverse to all the strata of $V$, and such that $W \cap V_{\beta}=\left(\Delta \cap V_{\beta}\right) \times U_{\alpha}$ for each stratum $V_{\beta}$ with $x \in \overline{V_{\beta}}$.

In his remarkable "Cargèse" article [263, B. Teissier introduced a decreasing sequence of numbers $\mu^{*}$, today known as the Teissier sequence, and proved that if a family has constant $\mu^{*}$-sequence, then it is Whitney equisingular. The converse, that Whitney equisingularity implies constant $\mu^{*}$-sequence was proved later by Briançon and Speder in [46] (cf. [45]). The $\mu^{*}$-sequence of an isolated hypersurface singularity $(X, \underline{0})$ in $\mathbb{C}^{n+1}$ is

$$
\mu^{*}:=\left\{\mu^{n+1}(X), \ldots, \mu^{i}(X), \ldots, \mu^{0}(X)\right\},
$$

where $\mu^{n+1}(X)=\mu(X)$ is the usual Milnor number, and $\mu^{i}(X)$ is the Milnor number of the intersection of $X$ with a general plane in $\mathbb{C}^{n+1}$ of dimension $i$, passing through $\underline{0}$.

There are several other concepts of equisingularity, for instance the Milnor equisingularity studied in [160], $\mu$-constant families as in Lê-Ramanujam's theorem [156, bi-Lipschitz equisingularity (see [197,228]), etc. The concept of equisingularity is also closely related to that of "simultaneous resolution" and work by Hironaka, Lipman, and others (see [264, p. 595]). 
Equisingularity has proved to be a very subtle subject, with a myriad of different aspects and open questions. For more on this subject, we refer to the literature, which is vast; see for instance the survey articles [120, 165] or [94, 96, 108, 175, 177].

10.1. Lipschitz geometry of singularities. Recall that a continuous map $f: Y \rightarrow Z$ between metric spaces $\left(Y, d_{Y}\right)$ and $\left(Z, d_{Z}\right)$ is Lipschitz if there is a constant $L \geq 1$ such that

$$
L^{-1} d_{Y}(a, b) \leq d_{Z}(f(a), f(b)) \leq L d_{Y}(a, b)
$$

for each pair of points $a, b \in X$. A metric space $Y$ admits local bi-Lipschitz parameterizations by $\mathbb{R}^{n}$ if every point in $Y$ has a neighborhood that is bi-Lipschitz homeomorphic to an open subset of $\mathbb{R}^{n}$. Two metric spaces $Y, Z$ are bi-Lipschitz equivalent if there is a bi-Lipschitz homeomorphism $Y \rightarrow Z$. Bi-Lipschitz classification is stronger than topological and weaker than $C^{\infty}$ classifications.

A topological $n$-manifold, $n \geq 2$, is a Lipschitz manifold if it can be equipped with an atlas where all transition functions are bi-Lipschitz. Such a manifold is smoothable if there is further a subsystem of charts where the transition functions are diffeomorphisms. A deep theorem of D. Sullivan in [260] guarantees that in dimensions $\neq 4$, there is a unique Lipschitz structure on every topological manifold [57], and this can be used to study the smoothability of topological manifolds, which is a deep theory explored in 254].

Bi-Lipschitz homeomorphisms have good properties that can be of interest in various fields of research, particularly for studying the geometry and topology of analytic spaces.

The study of bi-Lipschitz geometry of complex spaces started with Pham and Teissier in 233. Later, Mostowski in [196, 197] studied Lipschitz equisingularity and Lipschitz stratifications in analytic sets, a notion that grants the constancy of the Lipschitz type of the stratified set along each stratum. The existence of Lipschitz stratifications for complex analytic sets was established in [197, and in the real analytic case, including semianalytic sets, this was done by Parusiński in 228, 229. (see also 215]).

In [30] the authors look at Lipschitz properties of semialgebraic sets with singularities and study the concept of normal embeddings, which has opened an important line of research. For this, notice that given an analytic subset $X$ of $\mathbb{R}^{n}$, we have two natural metrics on $X$ : One is the metric induced from the ambient space; this is called the outer metric. The other is the inner, or length, metric defined in the usual way in differential geometry - as the infimum of lengths of piecewise smooth curves connecting two given points. The Lipschitz equivalence in terms of the outer metric is more rigid, and it implies the equivalence in an inner metric, but not inversely. The set $X$ is normally embedded if these two metrics are equivalent.

The main result in 30 states that every compact semialgebraic set is bi-Lipschitz equivalent to some normally embedded semialgebraic set. The article [27] started the study of the bi-Lipschitz geometry of complex surface singularities, and in recent years there has been remarkable progress in this and other related topics thanks to the work of L. Birbrair, A. Fernandes, A. Pichon, W. Neumann, G. Valette, D. Kerner, T. Gaffney, J. E. Sampaio, and many others (see for instance [26 29, 31, 98, 107, 139, 210, 214, 271, and the references therein).

Some of the important recent results in this area are the complete classification of the inner metrics of surfaces in [31]; the proof in [212] that Zariski equisingularity 
is equivalent to bi-Lipschitz triviality in the case of surfaces; the proof in [28] that outer Lipschitz regularity implies smoothness; and the important partial answer in 98] of Zariski's multiplicity conjecture (10.2), proving that if $f, g: \mathbb{C}^{n} \rightarrow \mathbb{C}$ are irreducible homogeneous polynomials such that there is a bi-Lipschitz homeomorphism $h:\left(\mathbb{C}^{n}, V(f), 0\right) \rightarrow\left(\mathbb{C}^{n}, V(g), 0\right)$, then $f$ and $g$ have the same multiplicity at 0 .

\section{Relations With OTHER BRANCHES}

11.1. Fibered knots and open-books. The concept of open-books was introduced by E. Winkelnkemper, and we refer to his appendix in 240] for a clear account of the subject. An open-book decomposition of a smooth $n$-manifold $M$ consists of a codimension 2 submanifold $N$, called the binding, embedded in $M$ with trivial normal bundle, together with a fiber bundle decomposition of its complement

$$
\theta: M-N \rightarrow \mathbb{S}^{1},
$$

satisfying that on a tubular neighborhood of $N$, diffeomorphic to $N \times \mathbb{D}^{2}$, the restriction of $\theta$ to $N \times\left(\mathbb{D}^{2}-\{0\}\right)$ is the map $(x, y) \mapsto y /\|y\|$. The fibers of $\theta$ are called the pages of the open-book. These are all diffeomorphic and each page $F$ can be compactified by attaching the binding $N$ as its boundary, thus getting a compact manifold with boundary.

Milnor's fibration theorem grants that in the isolated singularity case, we get open-book decompositions. In fact, given a complex analytic space $X$ which is nonsingular away from a point, say $\underline{0}$, and a holomorphic map-germ

$$
f:(X, \underline{0}) \longrightarrow(\mathbb{C}, 0),
$$

which is regular away from $\underline{0}$, by the fibration theorem one has an open-book decomposition on the link $L_{X}$ of $X$,

$$
\varphi:=\frac{f}{|f|}: L_{X} \backslash L_{f} \longrightarrow \mathbb{S}^{1},
$$

where $L_{f}$ is the link of $f$ in $X$ and the pages of the open-book are the Milnor fibers.

Recall that if $M$ is a smooth, closed, connected manifold, a knot in $M$ means a smooth codimension 2 closed, connected submanifold $N$ of $M$. If $N$ has several connected components then it is called a link in $M$; so the binding of an open-book is a knot (or link). The name "algebraic knot" was coined by Lê Dũng Tráng in [154 to characterize the knots defined by an algebraic (or analytic) equation with complex values.

In the literature there are also real algebraic knots. For instance Perron proved in 231. that the figure eight knot is real algebraic where, in analogy with the complex case, this means that it is defined by the link of a polynomial map $\mathbb{R}^{4} \rightarrow \mathbb{R}^{2}$ with an isolated critical point. In 8 the authors prove that "all knots are algebraic"; yet, one must be careful that the statement here is slightly different: although the codimension 2 real algebraic varieties that define the knots have an isolated singularity, the functions that define them may have nonisolated critical points. When this happens, the corresponding knot is not fibered: a knot (or link) $N \subset M$ is fibered if it is the binding of an open-book decomposition of $M$. The concept of fibered knots was introduced by A. Durfee and B. Lawson in 78, where they use Milnor fibrations to construct codimension 1 foliations on odd-dimensional spheres. By Milnor's theorem, every (complex) algebraic knot is fibered. 
The classical theory of one-dimensional knots in the 3-sphere which are algebraic goes back to Brauner [44]. The literature on this topic is vast, and we refer to the excellent book [48, for a clear account (see also [122, 276] and [193, Chapter 10]). Just a few words: every map-germ

$$
f:\left(\mathbb{C}^{2}, \underline{0}\right) \longrightarrow(\mathbb{C}, 0),
$$

has an essentially unique prime factorization $f=f_{1}^{a_{1}} \cdots f_{r}^{a_{r}}$. The zero locus $V(f)$ of $f$ is the union of the zero loci $V\left(f_{i}\right)$ of the $f_{i}$. Each of these defines a branch of $f$, an irreducible component of $V(f)$, and we would like to describe these branches.

As an example consider the complex polynomial $f\left(z_{1}, z_{2}\right) \rightarrow z^{p}+z^{q}$, for some $p, q>1$. Let $k$ be the greatest common divisor of $p, q$ and set $p^{\prime}=p / k, q^{\prime}=q / k$. Set $V=f^{-1}(0)$. Then $V$ has $k$ branches and the intersection of each branch with the unit sphere is a torus knot of type $\left(q^{\prime}, p^{\prime}\right)$, i.e., it is wrapped in a torus $S^{1} \times S^{1}$ so that it goes around a parallel $q^{\prime}$ times, and around a meridian $p^{\prime}$ times.

It is known in general that each branch of an analytic plane curve admits a particular type of parameterization called a Newton-Puiseux parameterization. This describes the link as an iterated torus knot, and the corresponding Puiseux pairs tell us exactly how to construct it (see [48] for details).

For $n=1$, the link of the singularity is either a circle or one circle for each branch, and the interesting point is knowing how these knots are embedded in the sphere. For $n>1$, the link itself has interesting topology. It is also interesting to study the links of holomorphic functions on complex surface singularities. These give knots and open-books in the link of the surface singularity, which is a 3-manifold (see for instance [234, 235, 237]).

11.2. Open-books and contact structures. Recall that if $M$ is an oriented $(2 n-1)$-dimensional manifold, a contact structure on $M$ is a hyperplane distribution $\zeta$ in its tangent bundle $T M$, which is locally given by a 1-form $\alpha$ such that $\alpha \wedge(d \alpha)^{(n-1)} \neq 0$. In this case we say that the pair $(M, \zeta)$ is a contact manifold and $\alpha$ is a contact form. The contact structure is called oriented if the vector bundle $\zeta$ is oriented. If $\alpha$ is a contact form, it is called positive if the volume form $\alpha \wedge(d \alpha)^{(n-1)}$ defines the orientation of $M$.

There is a natural way in which contact manifolds arise in complex geometry: Start with a complex manifold $X$ and a real hypersurface $M$ in it. At each point $z \in M$, we have the tangent spaces $T_{z} M \subset T_{z} X$. Multiplication of $T_{z} M$ by the complex number $i$ gives another real hyperplane $i\left(T_{z} M\right) \subset T_{z} X$. The intersection $\zeta_{z}:=T_{z} M \cap i\left(T_{z} M\right)$ is a real hyperplane in $T_{z} M$.

This hyperplane distribution in $M$ may or may not be a contact structure: if the real hypersurface $M$ in the complex manifold $X$ is strongly pseudoconvex, then the distribution $\zeta$ defined above is a naturally oriented contact structure (see for instance [239, Proposition 5.11]). Pseudoconvex means that $M$ can be defined locally, in a neighborhood of each of its points, as a regular level of a strictly plurisubharmonic function.

Consider now a complex analytic variety $X$ of pure dimension $n$ in some complex space $\mathbb{C}^{m}$, and let $p$ be an isolated singularity in $X$. Consider the intersection of $X$ with the spheres in $\mathbb{C}^{m}$ centered at $p$. Varchenko in [273] noticed that the square of the distance function restricted to $X$ is still a strictly plurisubharmonic function, so the link $L_{X}$ is pseudoconvex and it can be equipped with a natural contact structure as above. Furthermore, this contact structure on the link is 
independent of the choices of the embedding and of the (sufficiently small) spheres, up to contactomorphisms (well-defined up to isotopy). This leads to the following definition.

Definition 11.1. The oriented contact manifold associated in this way to every isolated singularity germ $(X, p)$, up to contactomorphisms isotopic to the identity, is called the contact boundary of $(X, p)$ and is denoted $(\partial(X, p), \xi(X, p))$.

An oriented contact manifold which is contactomorphic to the contact boundary of an isolated singularity is called Milnor fillable, a name introduced in [59] in reference to Milnor's work [193].

Every Milnor fillable contact manifold $(M, \xi)$ is holomorphically fillable, since every resolution of a singularity whose contact boundary $(\partial(X, p), \xi(X, p))$ is contactomorphic to $(M, \xi)$ gives a holomorphic filling of it. Moreover, if there is a singularity germ $(X, p)$ with contact boundary $(\partial(X, p), \xi(X, p))$ which is smoothable (see Definition (7.6), then it is easy to construct Stein representatives of its Milnor fibers, and these are Stein fillings of the contact boundary $(\partial(X, p), \xi(X, p))$ (see [239, Proposition 6.8]).

As pointed out in [239, p. 62] there is a remarkable difference between complex dimension 2 and higher dimensions which is highlighted by the following two theorems. First recall the Pham-Brieskorn singularities envisaged in section 1 . One knows from [4] that in the special case

$$
z_{0}^{2}+z_{1}^{2}+\cdots+z_{2 m-1}^{2}+z_{2 m}^{p}=0, \quad m \geq 2,
$$

with $p \equiv \pm 1(\bmod 8)$, the link is always diffeomorphic to the standard $(4 m-1)$ sphere. One has Ustilovsky's theorem in [270]:

Theorem 11.3. Varying $p \in \mathbb{N}$ in (11.2) above, the contact boundaries of the corresponding isolated hypersurface singularities are pairwise noncontactomorphic. One thus gets in this way infinitely many different contact structures on the $(4 m-1)$ sphere which are contact boundaries of some isolated complex hypersurface singularity.

In complex dimension 2 the situation is radically different, as was proved by Caubel, Némethi, and Popescu-Pampu in [59]:

Theorem 11.4. Every Milnor fillable oriented 3-manifold admits a unique Milnor fillable contact structure up to contactomorphism.

The proof of Theorem 11.4 uses work by E. Giroux in 109 that motivates the title for this subsection: the notion of contact structures carried by an open-book.

Definition 11.5. A positive contact structure $\xi$ on a closed oriented manifold $M$ is carried by an open-book $(N, \theta)$ if it admits a defining contact form $\alpha$ which verifies the following:

- $\alpha$ induces a positive contact structure on $N$;

- $d \alpha$ induces a positive symplectic structure on each fiber of $\theta$.

If a contact form $\alpha$ satisfies these conditions, then it is said to be adapted to $(N, \theta)$.

Giroux proved in [109] that on each three-dimensional closed oriented manifold, every contact structure is carried by some open-book, and two positive contact structures carried by the same open-book are isotopic. Thus, in order to describe a positive contact structure on a three-dimensional closed and oriented manifold, it 
is enough to describe an open-book which carries it. This is the strategy adopted in 59 to prove Theorem 11.4 .

There is another important line of research that springs from the following theorem of Eliashberg [91.

Theorem 11.6. Every Stein filling of the natural contact structure on $\mathbb{S}^{3}$ is diffeomorphic to the four-dimensional compact ball.

We may naturally ask when and how this theorem extends to the contact boundaries of normal complex surface singularities. For this we may want to characterize the Milnor fibers of a given isolated singularity (up to diffeomorphisms) amongst the fillings of the boundary of the singularity. Since the Milnor fibers of every smoothing can be choosen to be Stein fillings, it is natural to restrict our attention to the Stein fillings of the contact boundary. In complex dimension 2 one has Theorem 6.3, so in this case one is led to asking the following questions (see 239, Section $6.2])$.

- Is it possible to characterize the Milnor fibers of the various isolated surface singularities with a given topological type among the Stein fillings of the associated Milnor fillable contact 3-manifold?

- Are there situations in which one gets all the Stein fillings up to diffeomorphisms as such Milnor fibers?

There are several important contributions to this line of research done by various authors, and we refer to [239, Section 6.2] for an account of this. We finish this subsection with the following theorem from 204 that provides a generalization of Eliashberg's theorem, Theorem 11.6.

Theorem 11.7. The Milnor fibers of a cyclic quotient singularity exhaust the Stein fillings of its contact boundary up to diffeomorphism.

We refer to [239, Section 6] for a clear account of the subject discussed in this subsection, including a fairly complete bibliography and a list of interesting open questions.

11.3. Low-dimensional manifolds. If $(V, \underline{0})$ is a normal surface singularity in some affine space $\mathbb{C}^{N}$, then its link $L_{V}:=V \cap \mathbb{S}_{\varepsilon}$ is a closed oriented 3-manifold with a rich geometry. The interplay between 3 -manifold theory and complex surface singularities goes back to F. Klein [143] and many others; see for instance [72,73, 193, 194, 199, 207, 208. We refer to [249, Chapters 3, 4] for a thorough discussion on that subject.

For instance consider the polynomial map $\left(\mathbb{C}^{3}, \underline{0}\right) \stackrel{f}{\rightarrow}(\mathbb{C}, 0)$ given by

$$
\left(z_{1}, z_{2}, z_{3}\right) \mapsto z_{1}^{p}+z_{2}^{q}+z_{3}^{r}, \quad \text { with } p, q, r \geq 2 .
$$

The link is a three-dimensional Brieskorn manifold. Klein in 143 showed that when $1 / p+1 / q+1 / r>1$, the link is diffeomorphic to a quotient of $\mathbb{S}^{3}$ divided by a discrete subgroup. For instance for the triple $(2,2, r)$ we get the quotient $\mathbb{S}^{3} / \mathbb{Z}_{n}$ which is the lens space $L(n, 1)$. For the triple $(2,3,5)$, the group is the binary icosahedral group and $L_{f}$ is Poincaré's homology 3 -sphere.

Notice that if we order $p, q, r$ so that $p \leq q \leq r$, then the condition $1 / p+1 / q+$ $1 / r>1$ is satisfied only for the triples $(2,2, r)$, for every $r \geq 2,(2,3,3),(2,3,4)$, and $(2,3,5)$. In all cases the singularity we obtain is a rational double point, also called a Klein or Du Val singularity. 
For $1 / p+1 / q+1 / r=1$, the only possible triples up to permutation are $(2,3,6)$, $(2,4,4),(3,3,3)$; Milnor proved in [194 that the links of all these singularities are quotients of the three-dimensional Heisenberg group of all $3 \times 3$ matrices which are upper triangular, with 1's in the diagonal, divided by appropriate discrete subgroups. In all other cases we have $1 / p+1 / q+1 / r<1$, and the corresponding links are quotients of the universal cover $\widetilde{\mathrm{SL}}(2, \mathbb{R})$ of $\operatorname{PSL}(2, \mathbb{R})$ divided by the commutator of the lifting of the triangle group $\langle p, q, r\rangle \subset \operatorname{PSL}(2, \mathbb{R})$ to $\widetilde{\mathrm{SL}}(2, \mathbb{R})$. We refer to [194] or [249, Chapter 3] for details; see also [72, 73, 208].

In general, it follows from work by Grauert and Mumford and also (independently) $\mathrm{Du}$ Val, that an oriented closed 3-manifold $M$ is orientation preserving diffeomorphic to the link of an isolated complex surface singularity if and only if it is a Waldhausen manifold with negative definite intersection matrix (cf. [207]). The following is a classical open problem and, to my knowledge, there has not been any significant improvement in more than two decades (cf. [280]).

Problem 11.8. Characterize the Waldhausen manifolds (with negative definite intersection matrix) that appear as links of surface singularities in $\mathbb{C}^{3}$.

By 207] the orientation preserving homeomorphism type of the link $L_{V}$ of a normal surface singularity $(V, \underline{0})$ depends only on the analytic type of $(V, \underline{0})$. Hence every 3-manifold invariant is an invariant of singularities and, conversely, whatever invariant of 3-manifolds we want to understand, the links of surface singularities are a great source of examples.

It is interesting to study, in particular, the relations between topological invariants of the singularity, which can be determined from the link, and analytic invariants, for instance the Milnor number, the geometric genus, and the signature of the Milnor fiber.

This begins with M. Artin in 21,22, proving that the rational singularities are taut, i.e., they are characterized by the topology of the link. Laufer proved in [146] a formula for the Milnor number of hypersurface singularities in $\mathbb{C}^{3}$ using the Hirzebruch-Riemann-Roch theorem,

$$
\mu+1=\chi(\widetilde{V})+K_{\widetilde{V}}^{2}+12 \rho(V)
$$

where $\widetilde{V}$ is a good resolution, $\chi$ is its topological Euler characteristic, $K_{\widetilde{V}}^{2}$ is the self-intersection number of the canonical class of the resolution, and $\rho(V)$ is the geometric genus. This formula was extended in [257] to all smoothable Gorenstein surface singularities.

Notice that the right-hand side in Laufer's formula is well-defined for nonsmoothable singularities; this is called the Laufer invariant in 250. In fact that invariant splits into two parts, $\chi(\widetilde{V})+K_{\widetilde{V}}^{2}$ and $12 \rho(V, q)$. The first of these is topological, depending only on the topology of the link $L_{V}$. Notice that if $L_{V}$ is a rational homology sphere, then the first Betti number $b_{1}(\widetilde{V})$ of $\widetilde{V}$ vanishes and $\chi(\widetilde{V})+K_{\widetilde{V}}^{2}$ essentially coincides with the invariant in a conjecture by Némethi and Nicolaescu (see [202, Remark 4.8]; also [203, Subsection 2.4]) related to the Casson invariant conjecture that we state below.

Laufer's formula inspired Durfee's theorem in [76], which states that if a normal surface singularity $(V, p)$ is numerically Gorenstein, smoothable, and the complex tangent bundle of the Milnor fiber $F$ of a smoothing is trivial, then the signature 
of $F$ can be expressed as

$$
\sigma(F)=-\frac{1}{3}\left(2\left(\chi\left(V_{t}\right)-1\right)+K_{\widetilde{V}}^{2}+2 b_{1}(\widetilde{V})+b_{2}(\widetilde{V})\right) .
$$

The proof is based on Hirzebruch's signature theorem for closed oriented 4-manifolds. This formula was completed in [247] by proving [76, Conjecture 1.6], that the tangent bundle of the Milnor fiber of every smoothing of a Gorenstein surface singularity is trivial. Hence Durfee's formula applies to all Gorenstein smoothable singularities.

Just as Laufer's formula, Durfee's signature formula can be regarded as a consequence of the general Atiyah-Singer index theorem. It is then natural to ask what information about invariants such as the Milnor number, the signature of the Milnor fiber, and the geometric genus can be determined topologically. This brings us to [92, 247] and several important articles by Némethi et al.; see for instance 200, 203, 206.

Consider the minimal resolution $\pi: \widetilde{V} \rightarrow V$ of a Gorenstein surface singularity $(V, \underline{0})$, and let $K:=K_{\widetilde{V}}$ be a divisor of the canonical bundle $\mathcal{K}:=\mathcal{K}_{\widetilde{V}}$. Assume further (with no loss of generality) that the divisor $K$ is vertical; i.e., the support of the divisor is contained in the exceptional curve. For each vertical divisor $D \geq 0$, set $W=2 D-K_{\widetilde{V}}$. Such a divisor $W$ was called characteristic in [92, in analogy with the classical theory of characteristic vectors and submanifolds (see for instance [99, 142, 144]), because $W$ represents an integral homology class whose reduction modulo 2 essentially is the second Stiefel-Whitney class of $K_{\widetilde{V}}$. Then we have the equalities

$$
\operatorname{dim} H^{1}\left(\widetilde{V}, \mathcal{O}_{\widetilde{V}}\right)=\operatorname{dim} H^{0}\left(-K,\left.\mathcal{K}\right|_{K}\right)=\operatorname{dim} H^{0}\left(W,\left.\mathcal{D}\right|_{W}\right)+\frac{1}{8}\left(W^{2}-K_{\widetilde{V}}^{2}\right),
$$

where $\mathcal{D}$ is the line bundle of the divisor $D$. Since the first term above is the geometric genus and the last term in the right is obviously topological, it follows that $\rho_{g}$ is topological whenever we can find a characteristic divisor $W$ for which the integer $\operatorname{dim} H^{0}\left(W,\left.\mathcal{D}\right|_{W}\right)$ is topological.

The 3-manifolds which are links of complex surface singularities carry canonical contact and $\mathrm{Spin}^{\mathrm{c}}$ structures inherited from the holomorphic structure on $V$. If the singularity is Gorenstein, which includes all hypersurface and ICIS germs, then the link also has a Spin structure, canonical up to homotopy (by [250]). Assume the link is an integral homology sphere $\Sigma$, and let

$$
R(\Sigma)=\operatorname{Hom}^{*}\left(\pi_{1}(\Sigma), \mathrm{SU}(2)\right) /(\operatorname{ad~SU}(2))
$$

be the space of irreducible $\mathrm{SU}(2)$-representations of its fundamental group modulo conjugation. The space $R(\Sigma)$ is nondegenerate if it satisfies a certain condition for every $\alpha \in R(\Sigma)$ (see [262]). In this case $R(\Sigma)$ has finite cardinality and its Casson invariant is defined via a signed count of its points

$$
\lambda(\Sigma)=\frac{1}{2} \sum_{\alpha \in R(\Sigma)} \varepsilon_{\alpha}, \quad \text { with } \varepsilon_{\alpha}= \pm 1 .
$$

The integers $\varepsilon_{\alpha}= \pm 1$ are determined from an intersection theory associated with a Heegaard splitting of $\Sigma$. If $R(\Sigma)$ is degenerate, then it needs to be perturbed first to make it finite and then $\lambda(\Sigma)$ can be defined similarly; see [262. In [100] Fintushel and Stern proved that the Casson invariant of the Brieskorn homology 
spheres $\Sigma(p, q, r)$ is

$$
\lambda(\Sigma(p, q, r))=\frac{1}{8} \sigma(F(p, q, r)),
$$

where $F(p, q, r)$ is the Milnor fiber (see 69] for a geometric proof). This led to the Casson invariant conjecture stated in [209].

Conjecture 11.9. If $\Sigma$ is an integral homology sphere which is the link of an ICIS surface singularity, then its Casson invariant equals $1 / 8$ the signature of the Milnor fiber,

$$
\lambda(\Sigma)=\frac{1}{8} \sigma(F)
$$

This conjecture was proved in 209] for all weighted homogeneous surface singularities and for other families also, including the Brieskorn-Hamm complete intersections. Yet, the conjecture remains open.

This can also be regarded from the viewpoint of the Seiberg-Witten monopole equations (see [202, p. 282, 3.1]). The Seiberg-Witten invariant of a closed oriented 3 -manifold $M$ is a function SW from the set $\mathcal{S}(M)$ of $\operatorname{Spin}^{c}$-structures on $M$ to the integers $\mathbb{Z}$. Roughly speaking, this invariant counts the gauge equivalence classes of solutions to the Seiberg-Witten equations. In the case of homology spheres $\Sigma$ there is only one Spin $^{c}$-structure and, therefore, a single Seiberg-Witten invariant that we denote $\mathrm{SW}(\Sigma)$. In [198] is proved that for the Brieskorn homology spheres, this coincides with the Casson invariant

$$
\mathrm{SW}(\Sigma(p, q, r))=\lambda(\Sigma(p, q, r)) .
$$

It was then conjectured by P. Kronheimer that these two invariants coincide for all three-dimensional homology spheres. That conjecture is proved in 163 . Hence the Casson invariant conjecture can be studied in terms of Seiberg-Witten invariants. This line of research is being done by A. Némethi et al. (cf. [145, 201, 203, 206]). They also study conditions under which the geometric genus is a topological invariant.

In the previous discussion the surface singularities in question are isolated, so the link is a smooth 3-manifold, and if the singularity is an ICIS, then the link is isotopic to the boundary of the Milnor fiber. In [205] the authors consider the boundaries of Milnor fibers of nonisolated surface singularities in $\mathbb{C}^{3}$. These turn out to be Waldhausen manifolds as well, by [189, 190, 205. In 205] the authors give a way to determine the Waldhausen decomposition of these manifolds, and they thoroughly study their geometry and topology. The fact that the boundaries of the Milnor fibers of nonisolated complex hypersurfaces in $\mathbb{C}^{3}$ are Waldhausen manifolds is proved also in [97, where the authors extend that theorem to singularities defined by functions of the type $f \bar{g}$ with $f, g$ being holomorphic (see section 13).

\section{Part II. Beyond the Holomorphic Realm}

We now look at Milnor fibrations for real analytic singularities. This emerged too from Milnor's seminal work in [192,193. We also look at meromorphic functions, and remark that the much of the discussion below goes through for semialgebraic and subanalytic maps (see $70,79,80$ ). 


\section{Foundations AND First Steps}

The following was stated as Hypothesis 11.1 in 193 .

Definition 12.1. A real analytic map-germ $f:\left(\mathbb{R}^{n}, \underline{0}\right) \rightarrow\left(\mathbb{R}^{p}, 0\right), n \geq p>0$, satisfies the Milnor condition at $\underline{0}$ if the derivative $D f(x)$ has rank $p$ at every point $x \in U \backslash \underline{0}$, where $U$ is some open neighborhood of $\underline{0} \in \mathbb{R}^{n}$.

The following extends the fibration theorem to the real setting.

Theorem 12.2 (Milnor). Let $f$ satisfy Definition 12.1 at $\underline{0}$. For every $\varepsilon>0$ sufficiently small, let $\delta>0$ be sufficiently small with respect to $\varepsilon$, and consider the Milnor tube $N(\varepsilon, \delta):=f^{-1}\left(\partial \mathbb{D}_{\delta}\right) \cap \mathbb{B}_{\varepsilon}$, where $\mathbb{D}_{\delta}$ is the disc in $\mathbb{R}^{p}$ of radius $\delta$ and center at $0, \partial \mathbb{D}_{\delta}$ is its boundary, and $\mathbb{B}_{\varepsilon}$ is the closed ball in $\mathbb{R}^{n}$ of radius $\varepsilon$ and center $\underline{0}$. Then

$$
\left.f\right|_{N(\varepsilon, \delta)}: N(\varepsilon, \delta) \longrightarrow \partial \mathbb{D}_{\delta}
$$

is a fiber bundle. Moreover, the tube $N(\varepsilon, \delta)$ is diffeomorphic to

$$
\mathbb{S}_{\varepsilon}^{n-1} \backslash\left(f^{-1}\left(\stackrel{\circ}{D}_{\delta}\right) \cap \mathbb{S}_{\varepsilon}^{n-1}\right)
$$

where $\stackrel{\circ}{\mathbb{D}}_{\delta}$ is the interior, and (12.3) determines an equivalent fiber bundle

$$
\varphi: \mathbb{S}_{\varepsilon}^{n-1} \backslash L_{f} \rightarrow \mathbb{S}^{p-1},
$$

where $L_{f}=f^{-1}(0) \cap \mathbb{S}_{\varepsilon}^{n-1}$ is the link. The projection $\varphi$ is $f /\|f\|$ in a tubular neighborhood of $L_{f}$.

The statement that $\varphi=f /\|f\|$ in a tubular neighborhood of the link $L_{f}$ is implicit in Milnor's book, and it was made explicit in [66, 237]. The proof of (12.3) is an easy extension of the proof of Ehresmann's fibration lemma. As in the complex case, one then constructs an integrable vector field $v$ in the ball $\overline{\mathbb{B}}_{\varepsilon}$, which is transverse to all spheres in this ball centered at $\underline{0}$ and is transverse to all Milnor tubes. The integral curves of $v$ allow us to carry $N(\varepsilon, \delta)$ diffeomorphically into the complement of $f^{-1}\left(\mathbb{D}_{\delta}\right) \cap \mathbb{S}_{\varepsilon}^{n-1}$ in the sphere $\mathbb{S}_{\varepsilon}^{n-1}$, keeping its boundary fixed, and one extends the induced fibration to all of $\mathbb{S}_{\varepsilon}^{n-1} \backslash L_{f}$ using, for instance, that the normal bundle of the link is trivial.

Yet, we cannot in general inflate the tube in such a way that the projection $\varphi$ is $f /\|f\|$ everywhere. In fact this theorem has two weaknesses:

(1) It is much too stringent - map-germs satisfying Definition 12.1 are highly nongeneric.

(2) One has no control over the projection map $\varphi$ outside a neighborhood of the link.

Of course every complex-valued holomorphic function with an isolated critical point satisfies Definition 12.1 and does so also if we compose such a function with a real analytic local diffeomorphism of either the target or the source. The interesting point is finding examples which are honestly real analytic. Milnor exhibited the following examples in his book, suggested to him by N. Kuiper. Let $A$ denote either the complex numbers, the quaternions, or the Cayley numbers, and define

$$
h: A \times A \rightarrow A \times \mathbb{R},
$$


by $h(x, y)=\left(2 x \bar{y},|y|^{2}-|x|^{2}\right)$. Milnor first proves $([193,11.6])$ that this mapping carries the unit sphere of $A \times A$ to the unit sphere of $A \times \mathbb{R}$ by a Hopf fibration. Then he defines, more generally,

$$
f: A^{n} \times A^{n} \rightarrow A \times \mathbb{R},
$$

by

$$
f(x, y)=\left(2\langle x, y\rangle,\|y\|^{2}-\|x\|^{2}\right),
$$

where $\langle\cdot, \cdot\rangle$ is the Hermitian inner product in $A$. This map is a local submersion on a punctured neighborhood of $(0,0) \in A^{n} \times A^{n}$. The link of the corresponding singularity is the Stiefel manifold of 2 -frames in $A^{n}$ and the Milnor fiber is a disc bundle over the unit sphere of $A^{n}$.

For $p=1$, Definition 12.1 is always satisfied (see for instance 274]). For maps into $\mathbb{R}^{2}$, generically the critical values are real curves converging to $(0,0)$, though there are several families of singularities satisfying Definition 12.1, see for instance section 15. For $p>2$, few examples are known of map-germs satisfying Definition 12.1 and having a nontrivial Milnor fibration, where "nontrivial" means that the fibers are not discs.

There are in fact pairs $(n, p)$ as above for which no such examples exist, as stated in Theorem 12.6 below, as proved by Church and Lamotke in 61, and completing previous work by Looijenga in [166]:

Theorem 12.6. Let $n, p$ be positive integers.

(1) If $2 \geq n-p \geq 0$, then such examples exist for the pairs $\{(2,2),(4,3),(4,2)\}$.

(2) If $n-p=3$, nontrivial examples exist for $(5,2)$ and $(8,5) 6$ and perhaps for $(6,3)$.

(3) If $n-p \geq 4$, then such examples exist for all $(n, p)$.

In particular, if $p=2$, such examples exist for all $n \geq 4$. The case $(6,3)$ was left open and it was recently settled affirmatively in [15.

The proof in 61 follows the line in [166 and consists of an inductive process to decide for which pairs $(n, p)$ there exists a codimension $p$ submanifold $K$ of the sphere $\mathbb{S}^{n-1}$ with a tubular neighborhood $N$ which is a product $N \cong K \times D^{p}$, such that the natural projection $K \times\left(D^{p} \backslash\{0\}\right) \rightarrow \mathbb{S}^{p-1}$ given by $(x, y) \mapsto y /\|y\|$ extends to a smooth fiber bundle projection $\mathbb{S}_{n-1} \backslash K \rightarrow \mathbb{S}^{p-1}$. No explicit singularities satisfying the Milnor condition (Definition 12.1) were given.

The first explicit nontrivial example of a real analytic singularity with target $\mathbb{R}^{2}$ satisfying Definition 12.1] other than those in [193], was given by A'Campo [1]. This is the map $\mathbb{C}^{m+2} \rightarrow \mathbb{C}$ defined by

$$
\left(u, v, z_{1}, \ldots, z_{m}\right) \longmapsto u v(\bar{u}+\bar{v})+z_{1}^{2}+\cdots+z_{m}^{2} .
$$

The following notion was introduced in 241 .

Definition 12.7. Let $f:\left(\mathbb{R}^{n}, \underline{0}\right) \rightarrow\left(\mathbb{R}^{p}, 0\right), n>p \geq 2$, be analytic and satisfy the Milnor condition at $\underline{0}$, and let $L_{f}$ be its link. We say that $f$ satisfies the strong Milnor condition at $\underline{0}$ if for every sufficiently small sphere $\mathbb{S}_{\varepsilon}$ around $\underline{0}$,

$$
\frac{f}{|f|}: \mathbb{S}_{\varepsilon}-L_{f} \rightarrow \mathbb{S}^{p-1}
$$

is a fiber bundle. 
Jacquemard in 138 studied sufficient conditions to ensure that maps into $\mathbb{R}^{2}$ satisfying Definition 12.1 actually satisfy the strong Milnor condition. He gave two conditions which are sufficient but not necessary, and he constructs several examples of maps satisfying these conditions. The first of Jacquemard's conditions is geometric, the second condition is algebraic:

Condition A. There exists a neighborhood $U$ of the origin in $\mathbb{R}^{n}$ and a real number $0<\rho<1$ such that for all $x \in U-0$, one has

$$
\frac{\left|\left\langle\operatorname{grad} f_{1}(x), \operatorname{grad} f_{2}(x)\right\rangle\right|}{\left\|\operatorname{grad} f_{1}(x)\right\| \cdot\left\|\operatorname{grad} f_{2}(x)\right\|} \leq 1-\rho,
$$

where $\langle\cdot, \cdot\rangle$ is the usual inner product in $\mathbb{R}^{n}$.

Condition B. If $\varepsilon_{n}$ denotes the local ring of analytic map-germs at the origin in $\mathbb{R}^{n}$, then the integral closures in $\varepsilon_{n}$ of the ideals generated by the partial derivatives

$$
\left(\frac{\partial f_{1}}{\partial x_{1}}, \frac{\partial f_{1}}{\partial x_{2}}, \ldots, \frac{\partial f_{1}}{\partial x_{n}}\right) \text { and }\left(\frac{\partial f_{2}}{\partial x_{1}}, \frac{\partial f_{2}}{\partial x_{2}}, \ldots, \frac{\partial f_{2}}{\partial x_{n}}\right)
$$

coincide, where $f_{1}, f_{2}$ are the components of $f$.

One has the following.

Theorem 12.9 (Jacquemard). Let $f:\left(\mathbb{R}^{n}, \underline{0}\right) \rightarrow\left(\mathbb{R}^{2}, 0\right), n>2$, be an analytic map-germ. If the component functions $f_{1}$ and $f_{2}$ of $f$ satisfy Conditions $\mathrm{A}$ and $\mathrm{B}$, then for every sphere $\mathbb{S}_{\varepsilon}^{n-1}$ of radius $\varepsilon>0$ sufficiently small and centered at $\underline{0}$, one has that the projection map in (12.4),

$$
\varphi: \mathbb{S}_{\varepsilon}^{n-1} \backslash L_{f} \rightarrow \mathbb{S}^{1}
$$

can be taken to be $f /\|f\|$ everywhere.

Problem 12.10. What is the equivalent of Theorem 12.6 for the strong Milnor condition? That is, for which pairs $(n, p)$ does there exist an analytic map-germ $f:\left(\mathbb{R}^{n}, \underline{0}\right) \rightarrow\left(\mathbb{R}^{p}, 0\right), n>p \geq 2$, satisfying the strong Milnor condition at $\underline{0}$ ?

When $p=2$, such maps exist for all $n \geq 4$. There are also several examples with $p=3$ in 138 .

It was noted in 241 that Condition B can be relaxed and still have sufficient conditions to guarantee the strong Milnor condition. For this we recall the notion of the real integral closure of an ideal as given in [104].

Definition 12.11. Let $I$ be an ideal in the ring $\varepsilon_{m}$. The real integral closure of $I$, denoted by $\bar{I}_{\mathbb{R}}$, is the set of $h \in \varepsilon_{m}$ such that for all analytic $\varphi:(\mathbb{R}, 0) \rightarrow\left(\mathbb{R}^{m}, 0\right)$, we have $h \circ \varphi \in\left(\varphi^{*}(I)\right) \varepsilon_{1}$.

Given $f:\left(\mathbb{R}^{n}, 0\right) \rightarrow\left(\mathbb{R}^{2}, 0\right)$ as above, let us set the following.

Condition $\mathbf{B}_{\mathbb{R}}$. The real integral closures of the Jacobian ideals in (12.8) coincide.

For complex analytic germs, both conditions $B$ and $B_{\mathbb{R}}$ are equivalent (see [263], [104]). As pointed out in 241, essentially the same proof of Jacquemard in [138] gives the following.

Theorem 12.12. $h$ Let $f:\left(\mathbb{R}^{n}, 0\right) \rightarrow\left(\mathbb{R}^{2}, 0\right)$ be an analytic map-germ that satisfies the Milnor condition. If its components $f_{1}, f_{2}$ satisfy Conditions $\mathrm{A}$ and $\mathrm{B}_{\mathbb{R}}$, then $f$ satisfies the strong Milnor condition. 
This improvement of Theorem 12.9 was used in 241] to prove a stability theorem for real singularities with the strong Milnor condition. This was also used in 16 to find a theorem in the vein of Theorem 12.12 but using regularity conditions instead of Jacquemard's conditions. This inspired [241,248] and the use of the canonical pencil described in section 14. We refer for instance to [14, 15, 80, 82, 220, 222, 224, 236 for recent work on the topology of the Milnor fibers.

D. Massey in [180] improved Theorem 12.12 using a different viewpoint, via a generalized Lojasiewicz inequality (see section 13). We remark that Massey's viewpoint applies in a larger setting, which does not require $f$ to have an isolated critical point, and it significantly relaxes condition $\mathrm{B}_{\mathbb{R}}$.

\section{ON FUNCTIONS WITH A NONISOLATED CRITICAL POINT}

As noted before, considering map-germs $\left(\mathbb{R}^{n}, \underline{0}\right) \rightarrow\left(\mathbb{R}^{p}, 0\right)$ with an isolated critical point is very stringent. We now discuss the general case of arbitrary critical locus, starting with the slightly more general case of functions with an isolated critical value.

13.1. Functions with an isolated critical value. Every holomorphic map-germ $\left(\mathbb{C}^{m}, \underline{0}\right) \rightarrow(\mathbb{C}, 0)$ with a critical point at $\underline{0}$ has an isolated critical value, and the fibration theorems in Theorems 4.1 and 4.2 hold in this setting. It is thus natural to look for extensions of Milnor's theorem, Theorem 12.2, for analytic map-germs $f:=\left(f_{1}, \ldots, f_{p}\right):\left(\mathbb{R}^{n}, \underline{0}\right) \rightarrow\left(\mathbb{R}^{p}, 0\right)$ with a possibly nonisolated critical point at $\underline{0}$, such that $0 \in \mathbb{R}^{p}$ is the only critical value; i.e., all critical points of $f$ are in the special fiber $V:=f^{-1}(0)$. This was first done in [237.

Definition 13.1. If $f$ admits a fibration in tubes of the type (12.3), then we call this the (local) Milnor-Lê fibration of $f$ (or the Milnor fibration in tubes). If it admits a fibration on the spheres of the type in Definition 12.7, then we call this the (local) Milnor fibration of $f$ (or the Milnor fibration on spheres).

Given a real analytic map-germ $\left(\mathbb{R}^{n}, \underline{0}\right) \stackrel{f}{\rightarrow}\left(\mathbb{R}^{p}, 0\right), n>p \geq 1$, with an isolated critical value at $0 \in \mathbb{R}^{p}$, we want to know when is there a local Milnor-Lê fibration. That is, we want conditions to ensure that given a ball $\mathbb{B}_{\varepsilon}$ bounded by a Milnor sphere $\mathbb{S}_{\varepsilon}$ for $f$ (see Definition 3.3 ), there exists a ball $\mathbb{D}_{\delta}$ of some radius $\delta$ in $\mathbb{R}^{p}$, centered at 0 , such that if we set $N_{f}(\varepsilon, \delta):=f^{-1}\left(\mathbb{D}_{\delta} \backslash\{0\}\right) \cap \mathbb{B}_{\varepsilon}$, then

$$
\left.f\right|_{N_{f}(\varepsilon, \delta)}: N_{f}(\varepsilon, \delta) \longrightarrow \Delta_{\delta} \backslash\{0\}
$$

is a $C^{\infty}$ fiber bundle.

We know from 193 that this is always satisfied when $f$ has an isolated critical point (that is immediate from the implicit function theorem). Yet, when the critical point is not isolated; the situation is more delicate. In [237 it was noticed that if the map-germ $f$ is such that $V(f)=f^{-1}(0)$ has dimension $>0$ and $f$ has the Thom $a_{f}$-property, then $f$ has a local Milnor-Lê fibration.

The study of Milnor fibrations for real analytic map-germs was also addressed by D. Massey in 180. Recall that in 129. Hamm and Lê used the complex analytic Łojasiewicz inequality to show that Thom stratifications exist. Massey gives the appropriate generalization for the real analytic setting:

Definition 13.2. An analytic germ $\left(\mathbb{R}^{n}, \underline{0}\right) \stackrel{f}{\rightarrow}\left(\mathbb{R}^{2}, 0\right)$ satisfies the strong Eojasiewicz inequality at $\underline{0}$ if there exists a neighborhood $\mathcal{W}$ of $\underline{0}$ and constants $c, \theta \in \mathbb{R}$ 
with $c>0,0<\theta<1$, such that for all $x \in \mathcal{W}$ one has

$$
\|f(x)\|^{\theta} \leq c \min _{|(a, b)|=1}|a \nabla g(x)+b \nabla h(x)| .
$$

In this case the germ $f$ is said to be E-analytic.

The main theorem in [180] says the following.

Theorem 13.3 (Massey). If $f$ is E-analytic, then for every Milnor sphere $\mathbb{S}_{\varepsilon}$ there is a Milnor tube $N_{f}(\varepsilon, \delta)$ where $f$ is a proper stratified submersion and the projection of a $C^{\infty}$ fiber bundle. That is, E-analytic maps have Milnor-Lê fibrations.

Now we need the following definition from 68.

Definition 13.4. Let $f:\left(\mathbb{R}^{n}, \underline{0}\right) \stackrel{f}{\rightarrow}\left(\mathbb{R}^{p}, 0\right), n>p \geq 1$, have an isolated critical value at $0 \in \mathbb{R}^{p}$. We say that $f$ has the transversality property if for every sufficiently small sphere $\mathbb{S}_{\varepsilon}$ around the origin in $\mathbb{R}^{n}$, there exists $\delta>0$ such that all the fibers $f^{-1}(t)$ with $\|t\| \leq \delta$ meet the sphere $\mathbb{S}_{\varepsilon}$ transversally.

The transversality property appears already in [129, and in 224] this is called the Hamm-Lê condition. Maps with the Thom $a_{f}$-property and nonempty link have the transversality condition, but not conversely: there are examples by $\mathrm{M}$. Oka in 223 . of maps with the transversality property which do not have the Thom $a_{f}$-property (see also [188]).

The theorem below is Theorem 3.4 in [67. This improves [237, Theorem 1.3].

Theorem 13.5. Let $f:\left(\mathbb{R}^{n}, \underline{0}\right) \rightarrow\left(\mathbb{R}^{p}, 0\right), n>p \geq 1$, have an isolated critical value at $0 \in \mathbb{R}^{p}$. Assume further that $f$ has the transversality property and $V(f):=$ $f^{-1}(0)$ has dimension greater than 0 . Then $f$ has a local Milnor-Lê fibration

$$
\left.f\right|_{N_{f}(\varepsilon, \delta)}: N_{f}(\varepsilon, \delta) \longrightarrow \Delta_{\delta} \backslash\{0\},
$$

with $N_{f}(\varepsilon, \delta):=f^{-1}\left(\mathbb{D}_{\delta} \backslash\{0\}\right) \cap \mathbb{B}_{\varepsilon}$ for some ball $\mathbb{D}_{\delta} \subset \mathbb{R}^{p}, 0<\delta \ll \varepsilon$. This determines an equivalent fiber bundle

$$
\varphi:\left(\mathbb{S}_{\varepsilon} \backslash L_{f}\right) \longrightarrow \mathbb{S}^{p-1},
$$

where the projection map $\varphi$ is $f /\|f\|$ restricted to $\left[\mathbb{S}_{\varepsilon} \cap N(\eta, \delta)\right]$.

The way to pass from the fibrations in tubes to that on the sphere is as before: one constructs a smooth vector field $\zeta$ in the ball $\mathbb{B}_{\varepsilon}$ minus $V$, satisfying the following.

- Each integral line is transversal to all spheres in $\mathbb{B}_{\varepsilon}$ centered at 0 .

- Each integral line is transversal to all tubes $f^{-1}\left(\partial \mathbb{D}_{\delta}\right)$ contained in $\mathbb{B}_{\varepsilon}$.

The difference with the holomorphic setting is that we cannot now guarantee a third condition: that the vectors $f(z)$ are collinear for all points in each integral line (cf. Theorem 14.4 below). We discuss this in section 14.

For maps of the type $f \bar{g}$ that we envisage below, there is simple criterion in [97] and [188, Proposition 3.5] for deciding whether or not the map has the transversality property. This is called CT-regularity in [188. The advantage of this criterion is that it is easy to use in practice. 
13.2. Functions with arbitrary discriminant. We now consider the general setting and study Milnor fibrations for map-germs $\left(\mathbb{R}^{n}, \underline{0}\right) \rightarrow\left(\mathbb{R}^{p}, 0\right)$ with arbitrary critical points, following [63, 68]. We start with an example studied in [171] by López de Medrano.

Example 13.6. Consider maps $(f, g): \mathbb{R}^{n} \rightarrow \mathbb{R}^{2}$ the form

$$
(f, g)=\left(\sum_{i=1}^{n} a_{i} x_{i}^{2}, \sum_{i=1}^{n} b_{i} x_{i}^{2}\right),
$$

where the $a_{i}$ and $b_{i}$ are real constants in generic position in the Poincaré domain. This means that the origin is in the convex hull of the points $\lambda_{i}:=\left(a_{i}, b_{i}\right)$ and no two $\lambda_{i}$ are linearly dependent.

A simple calculation shows that $(f, g)$ is a complete intersection and the corresponding link is a smooth nonempty manifold of real codimension 2 in the sphere. The critical points $\Sigma$ of $(f, g)$ are the coordinate axis of $\mathbb{R}^{n}$, and the set $\Delta(f, g)$ of critical values is the union of the $n$ line segments in $\mathbb{R}^{2}$ joining the origin to the points $\lambda_{i}$. Hence $\mathbb{R}^{p}$ splits into various connected components, and it is proved in 171 that the topology of the fibers over points in different components changes. Yet, we know from [68] that these map-germs have the transversality property in Defintion 13.4 and away from the critical set they have a Milnor-Lê fibration. In fact these maps are d-regular too, a concept that we discuss in section 14 and which implies that away from the discriminant, they have also a Milnor fibration on small spheres with projection map $(f, g) /\|(f, g)\|$.

More generally, consider now an open neighborhood $U$ of $\underline{0} \in \mathbb{R}^{n}$ and a $C^{\ell}$ map $f:(U, \underline{0}) \rightarrow\left(\mathbb{R}^{p}, 0\right), n>p \geq 2, \ell \geq 1$, with a critical point at $\underline{0}$. Denote by $\mathcal{C}_{f}$ the set of critical points of $f$ in $\mathbb{B}_{\varepsilon}$, and let $\Delta_{f}$ be the image $f\left(\Delta_{f}\right)$. These are the critical values of $f$; we call $\Delta_{f}$ the discriminant of $f$.

Definition 13.7. We say that the map-germ $f$ has the transversality property at 0 if there exists a real number $\varepsilon_{0}>0$ such that, for every $\varepsilon$ with $0<\varepsilon \leq \varepsilon_{0}$, there exists a real number $\delta$, with $0<\delta \ll \varepsilon$, such that for every $t \in \mathbb{B}_{\delta}^{k} \backslash \Delta_{f}$, one has that either $f^{-1}(t)$ does not intersect the sphere $\mathbb{S}_{\varepsilon}^{n-1}$ or $f^{-1}(t)$ intersects $\mathbb{S}_{\varepsilon}^{n-1}$ transversally in $\mathbb{R}^{n}$.

The transversality condition of the fibers with small spheres ensures having a Milnor-Lê fibration, even for $C^{\ell}$ maps with nonisolated critical values. Of course, as in Example 13.6, if the base of the fibration has several connected components (sectors), then the topology of the fibers can change from one sector to another. We have the following result from [68].

Proposition 13.8. Let $f:\left(\mathbb{R}^{n}, \underline{0}\right) \rightarrow\left(\mathbb{R}^{p}, 0\right), n \geq p \geq 2$, be a map-germ of class $C^{\ell}$ with $\ell \geq 1$. If $f$ has the transversality property, then the restrictions

- $f \mid: \mathbb{B}_{\varepsilon}^{n} \cap f^{-1}\left(\mathbb{D}_{\delta}^{p} \backslash \Delta_{f}\right) \longrightarrow\left(\stackrel{\circ}{D}_{\delta}^{p} \backslash \Delta_{f}\right) \cap \operatorname{Im}(f)$,

- $f \mid: \mathbb{B}_{\varepsilon}^{n} \cap f^{-1}\left(\mathbb{S}_{\delta}^{p-1} \backslash \Delta_{f}\right) \longrightarrow\left(\mathbb{S}_{\delta}^{p-1} \backslash \Delta_{f}\right) \cap \operatorname{Im}(f)$

are $C^{\ell}$ fiber bundles, where $\varepsilon$ and $\delta$ are small enough positive real numbers, $\mathbb{B}_{\varepsilon}^{n} \subset \mathbb{R}^{n}$ and $\mathbb{D}_{\delta}^{p} \subset \mathbb{R}^{p}$ are the closed balls of radius $\varepsilon$ and $\delta$ centered at $\underline{0}$ and 0 , respectively, $\stackrel{\circ}{D}_{\delta}^{p}$ is the interior of the ball $\mathbb{D}_{\delta}^{p}$, and $\mathbb{S}_{\delta}^{p-1}$ is its boundary. If $f$ is analytic, then the fibrations above are $C^{\infty}$. 
In 63] the authors study the topology of the fibers of real analytic maps $\mathbb{R}^{n} \rightarrow$ $\mathbb{R}^{p+k}, n>p+k$, inspired by the classical Lề-Greuel formula for the Milnor number of isolated, complex, complete intersection germs. The idea is that if the map-germ is defined by analytic functions $\left(f_{1}, \ldots, f_{p}, g\right)$, then we can study the topology of its fibers by comparing it with the topology of the germ we get by dropping down $g$. We require for this that the map $f:=\left(f_{1}, \ldots, f_{p}\right)$ actually satisfies the Thom $a_{f}$-property with respect to some Whitney stratification $\left\{S_{\alpha}\right\}$ and that its zero-set $V(f)$ has dimension $\geq 2$ and it is a union of strata. The map-germ $\left(\mathbb{R}^{n}, \underline{0}\right) \stackrel{g}{\rightarrow}\left(\mathbb{R}^{k}, 0\right)$ is assumed to have an isolated critical point in $\mathbb{R}^{n}$ with respect to the stratification $\left\{S_{\alpha}\right\}$. By Proposition 13.8 the map-germs $f$ and $(f, g)$ have associated local MilnorLê fibrations. Then one has the corresponding Lê-Greuel formula [63, Theorem 1], as follows.

Theorem 13.9. Let $F_{f}$ and $F_{f, g}$ be Milnor fibers of $f$ and $(f, g)$ (any Milnor fibers, regardless of the fact that the topology of the fibers may depend on the connected component of the base once we remove the discriminant). Then one has

$$
\chi\left(F_{f}\right)=\chi\left(F_{f, g}\right)+\left.\operatorname{Ind}_{\mathrm{PH}} \nabla \tilde{g}\right|_{F_{f} \cap \mathbb{B}_{\varepsilon^{\prime}}},
$$

where $\tilde{g}: \mathbb{R}^{n} \rightarrow \mathbb{R}$ is given by $\tilde{g}(x)=\left\|g(x)-t_{0}\right\|^{2}$ with $t_{0} \in \mathbb{R}^{k}$ such that $F_{f, g}=$ $\left.g\right|_{F_{f}} ^{-1}\left(t_{0}\right)$ and $\mathbb{B}_{\varepsilon^{\prime}}$ is a small ball in $\mathbb{R}^{n}$ centered at the origin.

The term $\left.\operatorname{Ind}_{\mathrm{PH}} \nabla \tilde{g}\right|_{F_{f} \cap \mathbb{B}_{\varepsilon^{\prime}}}$ on the right, which by definition is the total PoincaréHopf index in $F_{f}$ of the vector field $\left.\nabla \tilde{g}\right|_{F_{f}}$, can be expressed also in the following equivalent ways:

(1) as the Euler class of the tangent bundle of $F_{f}$ relative to the vector field $\left.\nabla \tilde{g}\right|_{F_{f} \cap \mathbb{B}_{\varepsilon^{\prime}}}$ on its boundary;

(2) as a sum of polar multiplicities relative to $\tilde{g}$ on $F_{f} \cap \mathbb{B}_{\varepsilon^{\prime}}$;

(3) as the index of the gradient vector field of a map $\tilde{g}$ on $F_{f}$ associated to $g$;

(4) as the number of critical points of $\tilde{g}$ on $F_{f}$;

(5) when $p=1=k$, this invariant can also be expressed algebraically, as the signature of a certain bilinear form that originates from [17, 85, 89, 113,114 .

When $n=2 m, p=2 q, k=2$, and $\mathbb{C}^{m} \stackrel{(f, g)}{\longrightarrow} \mathbb{C}^{q+1}$ is holomorphic, this is a reformulation of the classical Lê-Greuel formula, Theorem 7.3 .

We remark that when $k=1$ and the germs of $f$ and $(f, g)$ are both ICIS germs, there is a Lê-Greuel type formula in 81] expressed in terms of normal data of $f$ with respect to an appropriate Whitney stratification. See also [187] for refinements of the above discussion.

\section{4. d-REgularity AND Milnor Fibrations}

The concept of d-regularity introduced in [66] is inspired by [16, 24, 241, 248, and it is a key for understanding the difference between real and complex singularities concerning Milnor fibrations.

14.1. The case of an isolated critical value. Let $U$ be an open neighborhood of the origin $\underline{0} \in \mathbb{R}^{n}$, and consider a real analytic germ $f:(U, \underline{0}) \rightarrow\left(\mathbb{R}^{p}, 0\right)$ which is a submersion for each $x \notin V:=f^{-1}(0)$ and has a critical point at $\underline{0}$.

Definition 14.1. The canonical pencil of $f$ is a family $\left\{X_{\ell}\right\}$ of real analytic spaces parameterized by $\mathbb{R} \mathbb{P}^{p-1}$ and defined as follows. For each $\ell \in \mathbb{R} \mathbb{P}^{p-1}$, consider the 
line $\mathcal{L}_{\ell} \subset \mathbb{R}^{p}$ that determines $\ell$, and set

$$
X_{\ell}=\left\{x \in U \mid f(x) \in \mathcal{L}_{\ell}\right\} .
$$

Note that every two distinct elements of the pencils $X_{\mathcal{L}}$ and $X_{\mathcal{L}^{\prime}}$ satisfy

$$
X_{\mathcal{L}} \cap X_{\mathcal{L}^{\prime}}=V .
$$

Each $X_{\mathcal{L}}$ has dimension $n-p+1$, is nonsingular outside $V$, and their union covers all of $U$.

Each line $\mathcal{L}$ intersects the sphere $\mathbb{S}^{p-1}$ in two antipodal points $\theta^{-}$and $\theta^{+}$. We decompose the line $\mathcal{L}$ into the corresponding half-lines accordingly:

$$
\mathcal{L}=\mathcal{L}^{-} \cup\{0\} \cup \mathcal{L}^{+} .
$$

If we define $E_{\theta \mp}$ to be the inverse image $f^{-1}\left(\mathcal{L}^{\mp}\right)$, respectively, then we can express each element of the canonical pencil as the union

$$
X_{\mathcal{L}}=E_{\theta^{-}} \cup V \cup E_{\theta^{+}} .
$$

If $L_{V}$ is the link of $f$, we can describe the fibers of the map $\varphi=f /\|f\|: \mathbb{S}_{\varepsilon}^{n-1} \backslash L_{V} \rightarrow$ $\mathbb{S}^{p-1}$ as

$$
\varphi^{-1}\left(\theta^{-}\right)=E_{\theta^{-}} \cap \mathbb{S}_{\varepsilon}^{n-1}, \quad \varphi^{-1}\left(\theta^{+}\right)=E_{\theta^{+}} \cap \mathbb{S}_{\varepsilon}^{n-1} .
$$

Then we can write

$$
X_{\mathcal{L}} \cap \mathbb{S}_{\varepsilon}^{n-1}=\left(E_{\theta^{-}} \cap \mathbb{S}_{\varepsilon}^{n-1}\right) \cup L_{V} \cup\left(E_{\theta^{+}} \cap \mathbb{S}_{\varepsilon}^{n-1}\right)=\varphi^{-1}\left(\theta^{-}\right) \cup L_{V} \cup \varphi^{-1}\left(\theta^{+}\right) .
$$

We now assume that $f: U \rightarrow \mathbb{R}^{p}$ is real analytic, with an isolated critical value at $\underline{0}$, and it is locally surjective, i.e., the restriction of $f$ to every neighborhood of $\underline{0} \in U$ covers a neighborhood of $0 \in \mathbb{R}^{p}$.

Definition 14.3. We say that $f$ is $d$-regular if there exists $\varepsilon_{0}>0$ such that for every $\varepsilon \leq \varepsilon_{0}$ and for every line $\mathcal{L}$ through the origin in $\mathbb{R}^{p}$, the sphere $\mathbb{S}_{\varepsilon}^{n-1}$ and the manifold $X_{\mathcal{L}} \backslash V$ are transverse.

As examples of d-regular maps one has the following.

- All holomorphic maps $\mathbb{C}^{n} \rightarrow \mathbb{C}$, all polar weighted homogeneous polynomials, and real weighted homogeneous maps with an isolated critical value are d-regular maps.

- If $f$ and $g$ are holomorphic maps $\mathbb{C}^{2} \rightarrow \mathbb{C}$ such that the product $f \bar{g}$ has an isolated critical value at the origin, then the map $f \bar{g}$ is d-regular, by [237].

- The strongly nondegenerate mixed functions in 221 are all d-regular, by 66, 221.

- Direct sums of d-regular maps; that is, if $f$ is d-regular in the variables $\left(x_{1}, \ldots, x_{n}\right)$ and $g$ is d-regular in the variables $\left(y_{1}, \ldots, y_{m}\right)$, then $f+g$ is d-regular in the variables $\left(x_{1}, \ldots, x_{n}, y_{1}, \ldots, y_{m}\right)$, by [66].

The following is a fundamental property of d-regularity. We refer to 66] for its proof.

Theorem 14.4. The real analytic map $f$ is d-regular if and only if there exists a smooth vector field $\zeta$ such that its integral lines are transverse to all spheres around $\underline{0}$, transverse to all Milnor tubes $f^{-1}\left(\partial \mathbb{D}_{\eta}\right) \cap \mathbb{B}_{\varepsilon}$, and tangent to each element $X_{\mathcal{L}}$ of the canonical pencil.

Such a vector field allows us to inflate the tube and get a fibration on the sphere minus the link, granting that the projection map is $f /\|f\|$. Hence we get the slight refinement of [66. Theorem 1] below. 
Theorem 14.5. Let $f:=\left(f_{1}, \ldots, f_{p}\right):\left(\mathbb{R}^{n}, \underline{0}\right) \rightarrow\left(\mathbb{R}^{p}, 0\right)$ be a locally surjective real analytic map with an isolated critical value at $0 \in \mathbb{R}^{p}$, and assume $V=f^{-1}(0)$ has dimension $>0$. Then $f$ admits a Milnor-Lê fibration if and only if it has the transversality property. If this is so, then $f$ is d-regular at 0 if and only if one has a commutative diagram of smooth fiber bundles

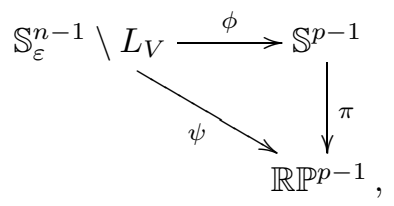

where $L_{V}$ is the link, $\psi=\left(f_{1}(x): \ldots: f_{p}(x)\right)$, and $\phi=\frac{f}{\|f\|}: \mathbb{S}_{\varepsilon}^{n-1} \backslash K_{\eta} \rightarrow \mathbb{S}^{p-1}$ is the Milnor fibration. Furthermore, if the two fibrations exist (one on a Milnor tube, another on the sphere minus the link), then these fibrations are smoothly equivalent. That is, there exists a diffeomorphism between their corresponding total spaces, carrying fibers into fibers.

This answers affirmatively a question raised by R. Araújo dos Santos in [13, where the author proved it for $p=2$ and $f$ weighted homogeneous. The proof in [66] of the equivalence of the two fibrations has a small gap that has been filled in 68] where the theorem is proved in the more general setting of real analytic maps with arbitrary linear discriminant; see Definition 14.7. In [66, 67] there are other criteria to determine d-regularity which can be useful in practice.

The following corollary is an immediate consequence of the previous theorem.

Corollary 14.6. Given $f:\left(\mathbb{R}^{n}, \underline{0}\right) \rightarrow\left(\mathbb{R}^{p}, 0\right)$ as in Theorem 14.5 , consider its Milnor fibration,

$$
\phi=\frac{f}{\|f\|}: \mathbb{S}_{\eta}^{n-1} \backslash K_{\eta} \rightarrow \mathbb{S}^{p-1} .
$$

Then the union of the link $K_{\eta}$ and each pair of fibers of $\phi$ over antipodal points of $\mathbb{S}^{p-1}$ corresponding to the line $\mathcal{L}_{\ell}$ is the link of the real analytic variety $X_{\theta}$.

For instance, if $f:\left(\mathbb{C}^{n}, 0\right) \rightarrow(\mathbb{C}, 0)$ is holomorphic and it has an isolated critical point at 0 , then $\{\operatorname{Re} f=0\}$ is a real hypersurface, and its link is the double of the Milnor fiber of $f$ with the link $L_{f}$ as an equator. If $n=2$, then the link of $\operatorname{Re}(f)=0$ is a compact Riemann surface of genus $2 g_{f}+r-1$, where $g_{f}$ is the genus of the Milnor fiber of $f$ and $r$ is the number of connected components of the link of $f$. Thus for instance, we know from [65] that for the map $\left(z_{1}, z_{2}\right) \stackrel{f}{\mapsto} z_{1}^{2}+z_{2}^{q}$, one gets that the link of Re $f$ is a closed oriented surface in the 3 -sphere, the union of the Milnor fibers over the points $\pm i$. An easy computation shows that it has genus $q-1$. This provides an explicit way to determine closed surfaces of all genera $\geq 1$ in the 3 -sphere by a single analytic equation.

It would be interesting to study the geometry and topology of the 4-manifolds one gets in this way, by considering the link of the hypersurface in $\mathbb{C}^{3}$ defined by the real part of a holomorphic function with an isolated critical point. For example, for the map $\left(z_{1}, z_{2}, z_{3}\right) \stackrel{f}{\mapsto} z_{1}^{2}+z_{2}^{3}+z_{3}^{5}$, the corresponding 4-manifold is the double of the $E_{8}$ manifold, whose boundary is Poincaré's homology 3-sphere. 
14.2. The general case. In 68 the authors continue the work begun in 63 and extend the above discussion on d-regularity to differentiable functions $\left(\mathbb{R}^{n}, 0\right) \stackrel{f}{\rightarrow}$ $\left(\mathbb{R}^{p}, 0\right)$ of class $\mathbb{C}^{\ell}, \ell \geq 1, n \geq p \geq 2$, with a critical point at $\underline{0} \in \mathbb{R}^{n}$, arbitrary critical values $\Delta_{f}$, and nonempty link $L_{V}$. This is immediate when the discriminant $\Delta_{f}$ is linear,

Definition 14.7. The map-germ $f:\left(\mathbb{R}^{n}, 0\right) \rightarrow\left(\mathbb{R}^{p}, 0\right)$ has linear discriminant if for some representative $f$ there exists $\eta=\eta(f)>0$ such that the intersection of $\Delta_{f}$ with the closed ball $\mathbb{D}_{\eta}^{p}$ is a union of line segments, i.e.,

$$
\Delta_{f} \cap \mathbb{D}_{\eta}^{p}=\operatorname{Cone}\left(\Delta_{f} \cap \mathbb{S}_{\eta}^{p-1}\right) .
$$

We call $\eta$ a linearity radius for $\Delta_{f}$. (The case where $f$ has $0 \in \mathbb{R}^{p}$ as an isolated critical value is considered to have linear discriminant with arbitrary linearity radius.)

Let $f:\left(\mathbb{R}^{n}, 0\right) \rightarrow\left(\mathbb{R}^{p}, 0\right)$ be as above, with linear discriminant, and consider a representative $f$ with linearity radius $\eta>0$. Set $\partial \Delta_{\eta}:=\Delta_{f} \cap \mathbb{S}_{\eta}^{p-1}$. For each point $\theta \in \mathbb{S}_{\eta}^{p-1}$, let $\mathcal{L}_{\theta} \subset \mathbb{R}^{p}$ be the open segment of line that starts at the origin and ends at the point $\theta$ (but which does not contain these two points). Set $E_{\theta}:=f^{-1}\left(\mathcal{L}_{\theta}\right)$, so each $E_{\theta}$ is a manifold of class $C^{\ell}$ for every $\theta$ in $\mathbb{S}_{\eta}^{p-1} \backslash \partial \Delta_{\eta}$.

Definition 14.8. Let $f:\left(\mathbb{R}^{n}, 0\right) \rightarrow\left(\mathbb{R}^{p}, 0\right)$ be a map-germ of class $C^{\ell}$ with $\ell \geq 1$ and linear discriminant. We say that $f$ is d-regular at 0 if for some representative $f$ there exists $\varepsilon_{0}>0$ small enough such that $f\left(\mathbb{B}_{\varepsilon_{0}}^{n}\right) \subset \mathbb{D}_{\eta}^{p}$, where $\eta$ is a linearity radius for $\Delta_{f}$, and such that every $E_{\theta}$ intersects the sphere $\mathbb{S}_{\varepsilon}^{n-1}$ transversally in $\mathbb{R}^{n}$, for every $\varepsilon$ with $0<\varepsilon \leq \varepsilon_{0}$ and for all $\theta \in \mathbb{S}_{\eta}^{p-1} \backslash \partial \Delta_{\eta}$ such that the intersection is not empty.

Example 14.9. Let $\mathcal{K}$ be either $\mathbb{R}$ or $\mathbb{C}$. Let $(f, g): \mathcal{K}^{n} \rightarrow \mathcal{K}^{2}$ be a $\mathcal{K}$-analytic map of the form

$$
(f, g)=\left(\sum_{i=1}^{n} a_{i} x_{i}^{q}, \sum_{i=1}^{n} b_{i} x_{i}^{q}\right),
$$

where $\left(a_{i}, b_{i}\right) \in \mathcal{K}$ are constants in generic position as in Example 13.6, and $q \geq 2$ is an integer. By 68 the discriminant $\Delta$ is linear and $(f, g)$ is d-regular.

It is proved in 68 that the fibration theorems, Theorems 14.4 and 14.5] extend to this general setting of $C^{\ell}$ maps with linear discriminant which have the transversality property and are d-regular. Also, in 68 there are examples of nonanalytic maps for which these fibration theorems apply.

Consider now the following example that generalizes Example 14.9. Let $(f, g): \mathbb{R}^{n} \rightarrow \mathbb{R}^{2}$ be

$$
(f, g)=\left(\sum_{i=1}^{n} a_{i} x_{i}^{p}, \sum_{i=1}^{n} b_{i} x_{i}^{q}\right),
$$

with $p, q \geq 2$ integers and the $\left(a_{i}, b_{i}\right)$ as above. If $p \neq q$, the discriminant $\Delta_{(f, g)}$ is not linear. Yet we can always linearize it with a homeomorphism $h$ in $\mathbb{R}^{2}$. Moreover, these maps have the transversality property and they are $\mathrm{d}_{h}$-regular in an appropriate sense that depends on the homeomorphism $h$. The fibration theorems in 68] extend to this setting, and in fact to all $C^{\ell}$-maps that admit an appropriate conic modification, a condition that seems to be always satisfied. 


\section{Mixed Singularities}

A mixed function is a real analytic function $\mathbb{C}^{n} \rightarrow \mathbb{C}$ in the complex variables $z=\left(z_{1},, z_{n}\right)$ and their conjugates $\bar{z}=\left(\bar{z}_{1},, \bar{z}_{n}\right)$. This type of function appeared in singularity theory already in [193, Chapter 11] as well as in the work of N. A'Campo [1] and Lee Rudolph 242. The modern study of mixed functions in singularity theory springs from [241,249]. The term "mixed singularity" was coined by M. Oka in 221.

15.1. Twisted Pham-Brieskorn singularities. The paradigm of real analytic functions with target $\mathbb{R}^{2}$ and satisfying the strong Milnor condition Definition 12.7 are the twisted Pham-Brieskorn singularities [249, Chapter VII],

$$
\left(z_{1}, \ldots, z_{n}\right) \stackrel{f}{\mapsto} z_{1}^{a_{1}} \bar{z}_{\sigma(1)}+\cdots+z_{n}^{a_{n}} \bar{z}_{\sigma(n)}, \quad a_{i} \geq 2,
$$

where $\sigma$ is a permutation of the set $\{1, \ldots, n\}$. It was noticed in [241, 248] that there exists a smooth action of $\mathbb{S}^{1} \times \mathbb{R}^{+}$on $\mathbb{C}^{n}$ of the form

$$
(\lambda, r) \cdot\left(z_{1}, \ldots, z_{n}\right)=\left(\lambda^{d_{1}} r^{p_{1}} z_{1}, \ldots, \lambda^{d_{n}} r^{p_{n}} z_{n}\right), \quad \lambda \in \mathbb{S}^{1}, r \in \mathbb{R}^{+},
$$

where the $d_{j}, p_{j}$ are positive integers such that $\operatorname{gcd}\left(d_{1}, \ldots, d_{n}\right)=1=\operatorname{gcd}\left(p_{1}, \ldots, p_{n}\right)$, and one has

$$
f\left((\lambda, r) \cdot\left(z_{1}, \ldots, z_{n}\right)\right)=\lambda^{d} r^{p} f\left(z_{1}, \ldots, z_{n}\right)
$$

for some positive integers $d, p$. So these are reminiscent of weighted homogeneous complex polynomials.

The basic properties are the following.

Lemma 15.1. The above $\mathbb{S}^{1}$-action on $\mathbb{C}^{n}$ has $V:=f^{-1}(0)$ as an invariant set, and it permutes the elements $X_{\theta}$ of the canonical pencil defined in section 14 .

Lemma 15.2. The orbits of the $\mathbb{R}^{+}$-action are transversal to every sphere around $0 \in \mathbb{C}^{n}$, except the orbit of $\underline{0} \in \mathbb{C}^{n}$, which is a fixed point, and all the orbits converge to $\underline{0}$ when the time tends to $-\infty$. This action leaves $V$ and each $X_{\theta}$ invariant.

The explicit weights for the above mentioned action of $\mathbb{S}^{1} \times \mathbb{R}^{+}$are easily computable from the exponents $a_{i}$ and the permutation $\sigma$ (see Lemmas 4.3 and 4.4 in Chapter VII of [249]).

The name twisted Pham-Brieskorn singularities comes from the similarity that these have with the classical Pham-Brieskorn polynomials (cf. section 21) and the fact, proved in 222,241 , that if the twisting $\sigma$ is the identity, then the corresponding open-books are equivalent to those of the usual Pham-Brieskorn singularities.

It was proved in [241,248 that these functions have an isolated critical point at $\underline{0}$, so they have a Milnor-Lê fibration in a tube, and in fact the local triviality of this bundle follows easily from the $\mathbb{S}^{1}$-action in Lemma 15.1. The first return map of this action is the monodromy map of the fibration. Then the flow given by Lemma 15.2 carries this fibration into one in the sphere with projection map $f /|f|$.

15.2. Polar weighted and radial weighted singularities. Consider, more generally, nonconstant polynomial maps $f: \mathbb{R}^{2 n} \rightarrow \mathbb{R}^{2}, n>1$, that carry the origin $\underline{0} \in \mathbb{R}^{2 n}$ into the origin $0 \in \mathbb{R}^{2}$. We identify the plane $\mathbb{R}^{2}$ with the complex line $\mathbb{C}$ and equip $\mathbb{C}^{*}$ with polar coordinates $\left\{r e^{i \theta} \mid r>0 ; \theta \in[0,2 \pi)\right\}$. We also identify $\mathbb{R}^{2 n}$ with $\mathbb{C}^{n}$ in the usual way. The following concept is introduced in 62 . 
Definition 15.3. The map $f$ is a polar weighted homogeneous polynomial if there exists an action of $\mathbb{S}^{1} \times \mathbb{R}^{+}$on $\mathbb{C}^{n}$ of the form

$$
(\lambda, r) \cdot\left(z_{1}, \ldots, z_{n}\right)=\left(\lambda^{d_{1}} r^{p_{1}} z_{1}, \ldots, \lambda^{d_{n}} r^{p_{n}} z_{n}\right), \quad \lambda \in \mathbb{S}^{1}, r \in \mathbb{R}^{+},
$$

where the $d_{j}, p_{j}$ are positive integers such that $\operatorname{gcd}\left(d_{1}, \ldots, d_{n}\right)=1=\operatorname{gcd}\left(p_{1}, \ldots, p_{n}\right)$, and one has

$$
f\left((\lambda, r) \cdot\left(z_{1}, \ldots, z_{n}\right)\right)=\lambda^{d} r^{p} f\left(z_{1}, \ldots, z_{n}\right),
$$

for some positive integers $d, p$. The $\mathbb{S}^{1}$-action is called a polar action while that of $\mathbb{R}^{+}$is a radial action.

This includes the twisted Pham-Brieskorn singularities as well as the weighted homogeneous complex polynomials. There are many other families (see [64, 220]). We remark that the polar action implies the critical value at $0 \in \mathbb{C}$, unless $f$ is constant.

Assuming that the dimension of $V=f^{-1}(0)$ is more than 0 , the Properties 1-4 in section 2 continue to hold in this setting. One has the canonical pencil as in section 14. Its elements $X_{\theta}$ are real algebraic hypersurfaces, smooth away from $V:=f^{-1}(0)$, they fill out the whole ambient space, and meet exactly at $V$. The $\mathbb{R}^{+}$action leaves invariant every element of the pencil, which therefore is transverse to all spheres around $\underline{0}$. And the orbits of the $\mathbb{S}^{1}$-action are tangent to all the spheres around $\underline{0}$ and permute the hypersurfaces $X_{\theta}$. Therefore, one has a global fibration as in equation (2.1), which restricts to the Milnor fibration Theorem 4.1 on each sphere, and by Property 2 this is equivalent to the fibration on a tube as in Theorem 4.2. The monodromy is the first return map of the $\mathbb{S}^{1}$-action.

In 64 the authors give a classification of the mixed homogeneous polynomials in three variables which are polar weighted with an isolated critical point. This generalizes classical work of Orlik and Wagreich on complex weighted homogeneous polynomials. In 135 137] there are interesting relations with contact structures and the enhaced Milnor number. And in 32, a special class of mixed singularities is used to show that certain braids can be compactified to become fibered real algebraic knots.

15.3. Meromorphic germs and the case $f \bar{g}$. Given two holomorphic map-germs $\mathbb{C}^{n} \stackrel{f, g}{\longrightarrow} \mathbb{C}$, we can associate to them:

- the meromorphic germ $f / g$;

- the real analytic map $f \bar{g}$, where $\bar{g}$ denotes complex conjugation.

Notice that the zero locus $V(f \bar{g})$ of $f \bar{g}$ equals, as a set, the zero locus of $f g$ and consists of $V(f) \cup V(g)$, the union of the zero loci of $f$ and $g$. Away from $V(f \bar{g})$ one has

$$
\frac{f / g}{|f / g|}=\frac{f \bar{g}}{|f \bar{g}|} .
$$

Therefore the two cases are equivalent when we look at Milnor fibrations with this map as projection. But they are very different when we look at the Milnor tubes; see Figure 6. We discuss first the case $f \bar{g}$.

The singular set of $V(f \bar{g})$ contains all points in $V(f) \cap V(g)$, which necessarily are critical points of $f \bar{g}$. Hence if $n>2$, this type of function must have nonisolated critical points. Yet, these may have an isolated critical value, as in the following 


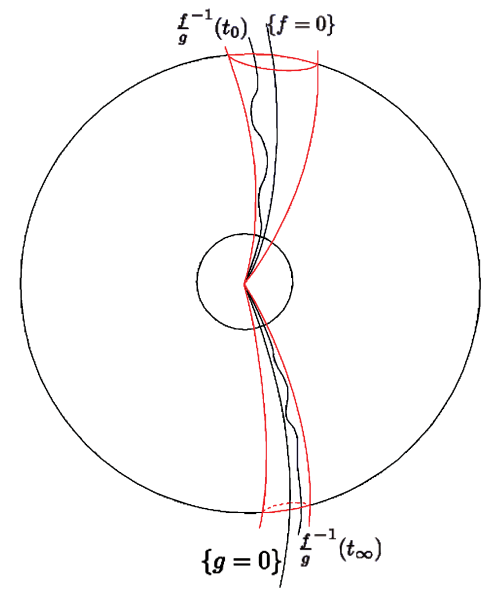

Figure 6. A Milnor tube $f / g=\left|t_{0}\right|$ for a meromorphic map.

examples from [237. Define $h: \mathbb{R}^{2 n} \rightarrow \mathbb{R}^{2}, n>1$, by $h=f \bar{g}$, where $g\left(z_{1}, \ldots, z_{n}\right)=$ $z_{1} \cdots z_{n}$ and $f$ is the Pham-Brieskorn polynomial

$$
f\left(z_{1}, \ldots, z_{n}\right)=\left(z_{1}^{a_{1}}+\cdots+z_{n}^{a_{n}}\right), \quad a_{i} \geq 2 .
$$

A straightforward computation shows that $h$ has an isolated critical value at $0 \in \mathbb{R}^{2}$ if and only if the $a_{i}$ satisfy $\sum_{i=1}^{n} 1 / a_{i} \neq 1$. For $n=2$ this means that at least one $a_{i}$ is more than 2. For $n=3$ the condition is that the unordered triple $(p, q, r)$ is not $(2,3,6),(2,4,4)$, or $(3,3,3)$.

In 235, 237 the authors study functions $f \bar{g}$ restricted to a complex analytic surface $X$ with (at most) an isolated singularity; of course $X$ can be $\mathbb{C}^{2}$. It is proved that these functions have the so-called Thom property, and this implies that one has a fibration of the Milnor-Lê type Theorem 4.2. Moreover, they prove that in this case one has a Milnor fibration

$$
f \bar{g} /|f \bar{g}|: \mathcal{L}_{X} \backslash L_{f \bar{g}} \rightarrow \mathbb{S}^{1}
$$

In [237, Theorem 5.2] they further look at the geometry of the fibration near the multilink $L_{f \bar{g}}$ in $\mathcal{L}_{X}$, and they prove the equivalence of the following three statements (we refer to 90 for background on fibered multilinks):

(i) the real analytic germ $f \bar{g}:(X, p) \rightarrow\left(\mathbb{R}^{2}, 0\right)$ has 0 as an isolated critical value;

(ii) the multilink $L_{f}-L_{g}$ is fibered; and

(iii) if $\pi: \tilde{X} \rightarrow X$ is a resolution of the holomorphic germ $f g:(X, p) \rightarrow(\mathbb{C}, 0)$, then for each rupture vertex $(j)$ of the decorated dual graph of $\pi$ one has $m_{j}^{f} \neq m_{j}^{g}$.

Moreover, it is proved that if these conditions hold, then the Milnor fibration in (15.5) actually is a fibration of the multilink $L_{f}-L_{g}$ (cf. [90]). This extends previous results in 235 for maps $\mathbb{C}^{2} \stackrel{f \bar{g}}{\rightarrow} \mathbb{C}$ to map-germs defined on surface singularities.

The Thom property is used in this setting to grant that the germ $f \bar{g}$ has the transversality property (Definition 13.4), and therefore it has a Milnor-Lê fibration. By 237. this holds for all maps $X \stackrel{f \bar{g}}{\rightarrow} \mathbb{C}$ where $X$ is a complex surface which 
is either regular or has a normal singularity. The same proof was generalized in 238 to higher dimensions, but this is mistaken and the erratum to that paper gives counterexamples. Yet, all the known counterexamples still satisfy the transversality property (see [188, 223]). This suggests a question.

Question 15.6. Do there exist germs $\mathbb{C}^{n} \stackrel{f \bar{g}}{\rightarrow} \mathbb{C}$ which do not have the transversality property, Definition 13.4? Can we classify them?

We now look at meromorphic germs. Let $U$ be an open neighborhood of $\underline{0}$ in $\mathbb{C}^{n}$, and let $f, g: U \longrightarrow \mathbb{C}$ be two holomorphic functions without common factors such that $f(\underline{0})=g(\underline{0})=0$.

Let us consider the meromorphic function $F=f / g: U \rightarrow \mathbb{C} P^{1}$ defined by $(f / g)(x)=[f(x) / g(x)]$. As in [125], two such germs at $\underline{0}, F=f / g$ and $F^{\prime}=f^{\prime} / g^{\prime}$, are considered as equal (or equivalent) if and only if $f=h f^{\prime}$ and $g=h g^{\prime}$ for some holomorphic germ $h: \mathbb{C}^{n} \rightarrow \mathbb{C}$ such that $h(\underline{0}) \neq 0$. Notice that $f / g$ is not defined on the whole $U$; its indetermination locus is

$$
I=\{z \in U \mid f(x)=0 \text { and } g(x)=0\} .
$$

In particular, the fibers of $F=f / g$ do not contain any point of $I$ : for each $c \in \mathbb{C}$, the fiber $F^{-1}(c)$ is

$$
F^{-1}(c)=\{x \in U \mid f(x)-c g(x)=0\} \backslash I .
$$

In a series of articles, Gusein-Zade, Luengo, and Melle-Hernández studied local Milnor-Lê type fibrations in Milnor tubes $f / g=|c|$ associated to every critical value of the meromorphic map $F=f / g$. See for instance [125, 126]. Of course these Milnor tubes are in fact pinched tubes that have $\underline{0}$ in their closure.

It is thus natural to ask whether one has for meromorphic map-germs Milnor fibrations on spheres, and if so, how these are related to those of the Milnor-Lê type, in Milnor tubes. The first of these questions was addressed in [33, 235, 237] from two different viewpoints, while the answer to the second question is the bulk of 34 where the authors compare the local fibrations in Milnor tubes of a meromorphic germ $f / g$, with the Milnor fibration in the sphere. They prove that if the germ $f / g$ satisfies two technical conditions (it is semitame and (IND)-tame), then the Milnor fibration for $f / g$ in the sphere is obtained from the Milnor-Lê fibrations of $f$ and $g$ in local tubes at $\underline{0}$ and $\infty$, by a gluing process that is "fiberwise", reminiscent of the classical connected sum of manifolds.

Remark 15.7. We know that if $(V, p)$ is a normal isolated complex surface singularity, then its link $L_{V}$ is a Waldhausen manifold, and there is a rich interaction between 3-manifolds theory and complex singularities (see section 11). When the germ $(V, p)$ is defined by a single equation $f: \mathbb{C}^{3} \rightarrow \mathbb{C}$, then $L_{V}$ can be regarded as the boundary of the Milnor fiber $F_{t}=f^{-1}(t) \cap \stackrel{\circ}{\mathbb{B}_{\varepsilon}}$. If we now consider a holomorphic map-germ $\mathbb{C}^{3} \rightarrow \mathbb{C}$ with a nonisolated critical point, then the corresponding link is no longer smooth, but we still have the Milnor fiber $F_{t}=f^{-1}(t) \cap \stackrel{\circ}{\mathbb{B}_{\varepsilon}}$, that can be regarded as a compact 4 -manifold by attaching to it its boundary $\partial F_{t}=f^{-1}(t) \cap \mathbb{S}_{\varepsilon}$. It is proved in [189, 190,205] that in this setting, the boundary of the Milnor fiber is also a Waldhausen 3-manifold. By [97, this statement actually extends to the boundary of the Milnor fibers of all map-germs in $\mathbb{C}^{3}$ of the form $f \bar{g}$ that have the transversality condition, which includes the holomorphic germs. 
15.4. A glance at Oka's work on mixed functions. Inspired by the theory of complex singularities, Oka in 221 introduces for mixed functions the useful notion of nondegeneracy with respect to a naturally defined Newton boundary. He uses this to prove a fibration theorem for strongly nondegenerate convenient mixed functions and to study their topology. We say a few words about this.

Let $f(z)$ be a mixed analytic function of the form

$$
f(z)=\sum_{\nu, \mu} c_{\nu, \mu} z^{\nu} \bar{z}^{\mu}
$$

where $\nu+\mu$ is the sum of the multi-indices of $z^{\nu} \bar{z}^{\mu}$, i.e.,

$$
\nu+\mu=\left(\nu_{1}+\mu_{1}, \ldots, \nu_{n}+\mu_{n}\right) .
$$

Assume for simplicity $c_{0,0}=0$, so that $\underline{0} \in V(f):=f^{-1}(0)$. Following Oka, we call $V(f)$ a mixed hypersurface, though in general it has real codimension 2 . The (radial) Newton polygon (at the origin) $\Gamma_{+}(f)$ is defined in the usual way: it is the convex hull of

$$
\bigcup_{c_{\nu, \mu} \neq 0}(\nu+\mu)+\mathbb{R}^{+n} .
$$

In analogy with complex polynomials, define the Newton boundary $\Gamma(f)$ as the union of the compact faces of $\Gamma_{+}(f)$. To every given positive integer (weight) vector $P=\left(p_{1}, \ldots, p_{n}\right)$, we associate a linear function $\ell_{P}$ on the Newton boundary $\Gamma(f)$ defined by

$$
\ell_{P}(\nu)=\sum_{j=1}^{n} p_{j} \nu_{j}
$$

for $\nu \in \Gamma(f)$. Let $\Delta(P, f)=\Delta(P)$ be the face where $\ell_{P}$ attains its minimal value. Then, for a positive weight $P$, define the face function $f_{P}(z)$ by

$$
f_{P}(z)=\sum_{\nu+\mu \in \Delta(P)} c_{\nu, \mu} z^{\nu} \bar{z}^{\mu} .
$$

Definition 15.8. Let $P$ be a strictly positive weight vector. We say that $f(z)$ is nondegenerate for $P$ if the fiber $f^{-1}(0) \cap \mathbb{C}^{* n}$ contains no critical point of the map $\mathbb{C}^{* n} \stackrel{f_{P}}{\rightarrow} \mathbb{C}$. The map $f$ is strongly nondegenerate for $P$ if the mapping $\mathbb{C}^{* n} \stackrel{f_{P}}{\rightarrow} \mathbb{C}$ has no critical points at all, $\operatorname{dim} \Delta(P) \geq 1$, and $f_{P}: \mathbb{C}^{* n} \rightarrow \mathbb{C}$ is surjective. The function $f(z)$ is called nondegenerate (resp., strongly nondegenerate) if it is nondegenerate (resp., strongly nondegenerate) for every strictly positive weight vector $P$.

For a subset $J \subset\{1,2, \ldots, n\}$, we consider the subspace $\mathbb{C}^{J}$ and the restriction $f^{J}:=\left.f\right|_{\mathbb{C}^{J}}$. Consider the set

$$
\mathcal{N} \mathcal{V}(f)=\left\{I \subset\{1, \ldots, n\} \mid f^{I} \neq 0\right\}
$$

We call $\mathcal{N} \mathcal{V}(f)$ the set of nonvanishing coordinate subspaces for $f$.

Definition 15.9. We say that $f$ is $k$-convenient if $J \in \mathcal{N} \mathcal{V}(f)$ for every $J \subset$ $\{1, \ldots, n\}$ with $|J|=n-k$. We say that $f$ is convenient if $f$ is $(n-1)$-convenient.

We may now state the main result in 221] concerning Milnor fibrations. In [221] this is stated as Theorems 29, 33, and 36; here we combine them into a single statement. 
Theorem 15.10. Assume the mixed polynomial $f(z)$ is convenient and strongly nondegenerate. Then one has a fibration of the Milnor-Lê type in a Milnor tube as in Theorem 4.2, as well as a Milnor fibration on every sufficiently small sphere with projection map $f /\|f\|$, as in Theorem 4.1, and the two fibrations are smoothly equivalent.

In 221 Oka also uses toric geometry to get a resolution of the corresponding singularity, in analogy with the complex case (see for instance the book [219]). He then uses this to study the topology of the links, as well as the topology of the Milnor fibers. Oka has subsequently studied and published various articles on the subject. We list some of them $(220223)$, but there are several more, with important results that cover a wide spectrum of topics, from intersection theory to contact structures.

\section{LinEAR ACTIONS, INTERSECTIONS OF QUADRICS, AND LVM MANIFOLDS}

The motivation in 248 for studying the twisted Pham-Brieskorn singularities comes from the pioneering work 169 by S. López de Medrano on intersections of quadrics and the space of Siegel leaves of holomorphic linear flows. In fact, notice that if $\xi$ is the holomorphic vector field in $\mathbb{C}^{n}$ defined by

$$
\xi(z)=\left(\lambda_{1} z_{\sigma(1)}^{a_{1}}, \ldots, \lambda_{n} z_{\sigma(n)}^{a_{n}}\right), \quad \lambda_{i} \in \mathbb{C}^{*},
$$

where $\sigma$ is a permutation of the set $\{1, \ldots, n\}$, then the set of points where the Hermitian product $\langle\xi(z), z\rangle$ vanishes is the mixed variety in $\mathbb{C}^{n}$ defined by

$$
V_{\xi}:=\left\{\lambda_{1} z_{\sigma(1)}^{a_{1}} \bar{z}_{1}+\cdots+\lambda_{n} z_{\sigma(n)}^{a_{n}} \bar{z}_{n}=0\right\} .
$$

Relabelling the variables $\left(z_{1}, \ldots, z_{n}\right)$, and assuming all $a_{i}>1$, we arrive at the twisted Pham-Brieskorn polynomials (up to the $\lambda_{i}$ 's). Notice that the variety defined in (16.1) is the set of points where the leaves (or solutions) of the vector field $\xi$ are tangent to the foliation in $\mathbb{C}^{n}$ by all the spheres centered at $\underline{0}$, union $\underline{0}$ itself.

If the exponents $a_{i}$ are all 1 , the vector field is linear. And if the twisting $\sigma$ is the identity, then $\xi$ is a linear diagonal vector field with eigenvalues $\lambda_{1}, \ldots, \lambda_{n}$. In this case one has

$$
\langle\xi(z), z\rangle=\sum_{i=1}^{n} \lambda_{i}\left|z_{i}\right|^{2},
$$

and the variety $V_{\xi}$ in (16.1) is the intersection of the two real quadrics below, corresponding to the real and the imaginary parts

$$
\operatorname{Re}\left(\sum_{i=1}^{n} \lambda_{i}\left|z_{i}\right|^{2}\right)=0 \quad \text { and } \quad \operatorname{Im}\left(\sum_{i=1}^{n} \lambda_{i}\left|z_{i}\right|^{2}\right)=0 .
$$

We say that $\xi$ is in the Siegel domain if the convex hull of the $\lambda_{i}$ in $\mathbb{C}$ contains the origin $\underline{0}$; otherwise $\xi$ is in the Poincaré domain; see Figure 7

When $\xi$ is in the Poincaré domain, it is easy to show that the variety $V_{\xi}$ actually consists only of the point $\underline{0}$. So we will restrict our discussion from now on to linear vector fields in the Siegel domain.

It is known that the equality, and even real dependence, of two eigenvalues of $F$ complicates the topology of $\mathcal{F}$ and $V_{F}$ very much. Therefore, one usually assumes 

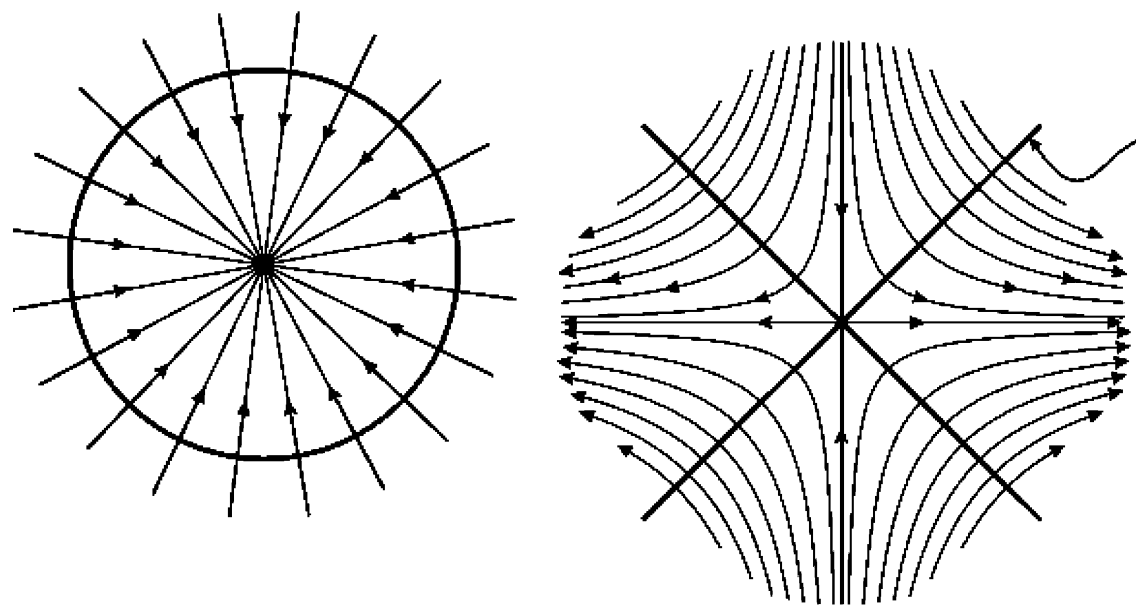

FiguRE 7. Linear vector fields in the Poincaré and Siegel domains, respectively. The curved arrow points into the space of Siegel leaves.

the following generic hyperbolicity hypothesis. Any two eigenvalues are independent over $\mathbb{R}$ :

$$
i \neq j \Rightarrow \lambda_{i} \notin \mathbb{R} \lambda_{j} \quad \forall i, j=1, \ldots, n
$$

So we now let $F$ be a linear vector field in the Siegel domain satisfying (16.2). It is clear that a point $z \in \mathbb{C}^{n}-0$ is in $V_{F}$ iff the restriction of the real function $d(z)=$ $\|z\|^{2}=\sum_{i=1}^{n}\left|z_{i}\right|^{2}$ to the leaf $\mathcal{L}_{z}$ through $z$ has a critical point at $z$. Furthermore, as noted in [57, Section 3], the fact that the solutions of $F$ are parameterized by exponential maps implies that the leaves of $F$ are concave. Thus, if a leaf $\mathcal{L}$ meets $V_{F}^{*}$, then it has a unique point in $V_{F}$, and it is the point in $\mathcal{L}$ of minimal distance to $0 \in \mathbb{C}^{n}$. Such a leaf is called a Siegel leaf. It is a copy of $\mathbb{C}$ embedded in $\mathbb{C}^{n}$ and can be characterized by its unique point in $V_{F}$. Furthermore, the fact that the intersection $\mathcal{L} \cap V_{F}$ of each leaf that meets $V_{F}$ is at a local minimal point in $\mathcal{L}$, implies that $\mathcal{L} \cap V_{F}$ is a transverse intersection. By the flow-box theorem for complex differential equations, this means that we have at each $z \in V_{F}^{*}$ a neighborhood of the form $U_{z} \times \mathbb{D}^{2}$, where $U_{z}$ is a disc of real dimension $2 n-2$ and the second factor denotes small discs in the leaves. It follows that $V_{F}^{*}$ is a smooth real submanifold of $\mathbb{C}^{n}$ of codimension 2 . In fact $V_{F}$ is a real analytic complete intersection in $\mathbb{C}^{n}$, and the union $W=V_{F}^{*} \times \mathbb{C}$ of all the Siegel leaves of $F$ is an open subset of $\mathbb{C}^{n}$ that can be identified with the total space of the normal bundle of $V_{F}^{*}$.

It is shown in [57] that $W$ is actually dense in $\mathbb{C}^{n}$. It is an exercise to see that $V_{F}$ is globally embedded as a cone with vertex at 0 and base the intersection $M=V_{F} \cap \mathbb{S}^{2 n-1}$ with the unit sphere, which is the link of the corresponding singularity.

We summarize part of the above discussion in the following theorem, which rephrases results in [57]. 
Theorem 16.3. Let $F(z)=\left(\lambda_{1} z_{1}, \ldots, \lambda_{n} z_{n}\right)$ be a linear vector field in the Siegel domain satisfying the hyperbolicity condition (16.2). Then the real analytic variety

$$
V_{F}=\left\{z \in \mathbb{C}^{n} \mid \operatorname{Re}\left(\sum_{i=1}^{n} \lambda_{i}\left|z_{i}\right|^{2}\right)=0\right\} \cap\left\{z \in \mathbb{C}^{n} \mid \operatorname{Im}\left(\sum_{i=1}^{n} \lambda_{i}\left|z_{i}\right|^{2}\right)=0\right\},
$$

is a complete intersection of real codimension 2 with an isolated singularity at 0 , and the regular points of $V_{F}$ parameterize the Siegel leaves of $F$.

Notice that one has the mixed function defined by $\left(\operatorname{Re}\left(\sum_{i=1}^{n} \lambda_{i}\left|z_{i}\right|^{2}\right)\right.$, $\left.\operatorname{Im}\left(\sum_{i=1}^{n} \lambda_{i}\left|z_{i}\right|^{2}\right)\right)$, which determines the complete intersection germ in Theorem 16.3. It is easy to see that its discriminant $\Delta_{F}$ consists of the $n$ half-lines in $\mathbb{C}$ determined by the eigenvalues $\lambda_{i}$. These split $\mathbb{C}$ into $n$-sectors and, just as in Example 13.6. one has a Milnor-Lê fibration over each sector. The topology of the Milnor fibers over the various sectors is determined in [171]. In [172] it is shown that the fibers over points in the discriminant necessarily are singular. This is related with a problem studied by C. T. C. Wall 275$]$ in relation with the topological stability of smooth mappings.

The topology of the link of these singularities is studied in [57] when $n=3$ and in $\left[169,170\right.$, for $n>3$. For $n=3$, the link is always a 3 -torus $\mathbb{S}^{1} \times \mathbb{S}^{1} \times \mathbb{S}^{1}$. For $n>3$, the link is always a connected sum of products of spheres determined by the geometry of the polytope spanned by the eigenvalues of $F$.

These constructions extend to sets of $m$ commuting vector fields on $\mathbb{C}^{n}, m \ll n$. This is studied in 173, 184, 185. A remarkable point is that in all these cases, the manifold $V_{F}^{*}:=V_{F} \backslash\{0\}$ has a canonical complex structure. In fact, under the appropriate hyperbolicity condition, $V_{F}^{*}$ is a smooth submanifold of $\mathbb{C}^{n}$ of real codimension $2 m$; the leaves of $\mathcal{F}$ are transversal to $V_{F}^{*}$ everywhere, and this equips $V_{F}^{*}$ with a canonical complex structure, by a theorem of Haefliger in 127. We remark that $V_{F}^{*}$ is not embedded in $\mathbb{C}^{n}$ as a complex submanifold.

This all is very surprising: for instance, consider the case $n=3$ and $m=1$. One has that $V_{F}$ is a real analytic singularity with a canonical complex structure away from 0 and the link is $\mathbb{S}^{1} \times \mathbb{S}^{1} \times \mathbb{S}^{1}$. Then $V_{F}$ is a real analytic complete intersection with a canonical complex structure on its regular part $V_{F}^{*}$, but $V_{F}$ cannot be complex at 0 because the 3 -torus cannot be the link of a complex singularity, by 259.

The complex manifolds $V_{F}^{*}$ one gets in this way are equipped with a canonical $\mathbb{C}^{*}$-action, and the quotient $V_{F}^{*} / \mathbb{C}^{*}$ is a compact complex manifold. These are known as LVM-manifolds (see [186]), and they are a special class of the so-called moment-angle manifolds, with remarkable geometric and topological properties (cf. 23,110 ).

It is also interesting to determine how the topology of the singularities defined as above varies as we pass from one component in the Siegel domain to another one, i.e., as we break the weak hyperbolicity condition. This is beautifully answered in [35. This is a wall-crossing problem as the authors explain, and they show that crossing a wall means performing a precise surgery, which they describe. From the viewpoint of singularities, what we do when crossing a wall is to put two complete intersection singularities in a one-parameter family of singularities which are all complete intersections, except that they bifurcate when crossing the wall. 


\section{ABOut THE AUthor}

José Seade works at the Mathematics Institute of the National University of Mexico (UNAM), where he currently is Director. He has published research papers and monographs with various authors on several aspects of real and complex singularities and on discrete group actions on complex spaces.

\section{ACKNOWLEDGMENTS}

I am happy to thank Patrick Popescu-Pampu, Bernard Teissier, Lê Dũng Tráng, Javier Fernándes de Bobadilla, David Massey, Jawad Snoussi, José Luis Cisneros, Roberto Callejas-Bedregal, Michelle Morgado, and Aurelio Menegon for wonderful conversations and useful comments that greatly enriched this presentation. I am also indebted to Martin Guest for reading the manuscript and making many helpful remarks, and to the referee, whose comments made this article far more readable.

\section{REFERENCES}

[1] N. A'Campo, Le nombre de Lefschetz d'une monodromie (French), Nederl. Akad. Wetensch. Proc. Ser. A 76 = Indag. Math. 35 (1973), 113-118. MR0320364 1284 299 318 327

[2] N. A'Campo, La fonction zêta d'une monodromie (French), Comment. Math. Helv. 50 (1975), 233-248, DOI 10.1007/BF02565748. MR0371889 299

[3] N. A'Campo, Structures de pseudovariété sur les espaces analytiques complexes (French), Intersection cohomology (Bern, 1983), Progr. Math., vol. 50, Birkhäuser Boston, Boston, MA, 1984, pp. 41-45, DOI 10.1007/978-0-8176-4765-0_4. MR788175 1308

[4] M. A. Aguilar, J. A. Seade, and A. Verjovsky, Indices of vector fields and topological invariants of real analytic singularities, J. Reine Angew. Math. 504 (1998), 159-176. MR.1656755 1302

[5] H. Aguilar-Cabrera, New open-book decompositions in singularity theory, Geom. Dedicata 158 (2012), 87-108, DOI 10.1007/s10711-011-9622-z. MR.2922705 1284

[6] H. Aguilar-Cabrera, Open-book decompositions of $\mathbb{S}^{5}$ and real singularities, Internat. J. Math. 25 (2014), no. 9, 1450085, 17, DOI 10.1142/S0129167X14500852. MR3266528 1284

[7] H. Aguilar-Cabrera, The topology of real suspension singularities of type $f \bar{g}+z^{n}$, Math. Z. 277 (2014), no. 1-2, 209-240, DOI 10.1007/s00209-013-1251-9. MR.3205769 284

[8] S. Akbulut and H. King, All knots are algebraic, Comment. Math. Helv. 56 (1981), no. 3, 339-351, DOI 10.1007/BF02566217. MR639356 1310

[9] P. Aluffi, Chern classes for singular hypersurfaces, Trans. Amer. Math. Soc. 351 (1999), no. 10, 3989-4026, DOI 10.1090/S0002-9947-99-02256-4. MR.1697199 «306

[10] P. Aluffi, Inclusion-exclusion and Segre classes, Comm. Algebra 31 (2003), no. 8, 3619-3630, DOI 10.1081/AGB-120022435. Special issue in honor of Steven L. Kleiman. MR2007377 306

[11] P. Aluffi and M. Marcolli, Feynman motives of banana graphs, Commun. Number Theory Phys. 3 (2009), no. 1, 1-57, DOI 10.4310/CNTP.2009.v3.n1.a1. MR.2504753 1306

[12] A. Andreotti and T. Frankel, The Lefschetz theorem on hyperplane sections, Ann. of Math. (2) 69 (1959), 713-717, DOI 10.2307/1970034. MR0177422 1296

[13] R. N. Araújo dos Santos, Equivalence of real Milnor fibrations for quasi-homogeneous singularities, Rocky Mountain J. Math. 42 (2012), no. 2, 439-449, DOI 10.1216/RMJ-2012-422-439. MR2915500 325

[14] R. Araújo dos Santos, D. Dreibelbis, and N. Dutertre, Topology of the real Milnor fiber for isolated singularities, Real and complex singularities, Contemp. Math., vol. 569, Amer. Math. Soc., Providence, RI, 2012, pp. 67-75, DOI 10.1090/conm/569/11242. MR/2934590 320

[15] R. Araújo Dos Santos, M. A. B. Hohlenwerger, O. Saeki, and T. O. Souza, New examples of Neuwirth-Stallings pairs and non-trivial real Milnor fibrations (English, with English and French summaries), Ann. Inst. Fourier (Grenoble) 66 (2016), no. 1, 83-104. MR.3477871 $1 3 1 8 \longdiv { 3 2 0 }$ 
[16] R. N. Araújo dos Santos and M. A. S. Ruas, Real Milnor fibrations and (c)-regularity, Manuscripta Math. 117 (2005), no. 2, 207-218, DOI 10.1007/s00229-005-0555-4. MR.2150482 1320,323

[17] V. I. Arnol'd, The index of a singular point of a vector field, the Petrovskiǔ-Oleinik inequalities, and mixed Hodge structures (Russian), Funkcional. Anal. i Priložen. 12 (1978), no. 1, 1-14. MR 04985921323

[18] V. I. Arnol'd, Catastrophe theory, Springer-Verlag, Berlin, 1984. Translated from the Russian by R. K. Thomas. MR.727639 1285

[19] E. Artal Bartolo, P. Cassou-Noguès, I. Luengo, and A. Melle Hernández, Quasi-ordinary power series and their zeta functions, Mem. Amer. Math. Soc. 178 (2005), no. 841, vi+85, DOI 10.1090/memo/0841. MR2172403 1299

[20] E. Artal Bartolo, I. Luengo, and A. Melle-Hernández, Milnor number at infinity, topology and Newton boundary of a polynomial function, Math. Z. 233 (2000), no. 4, 679-696, DOI 10.1007/s002090050493. MR1759267 300

[21] M. Artin, Some numerical criteria for contractability of curves on algebraic surfaces, Amer. J. Math. 84 (1962), 485-496, DOI 10.2307/2372985. MR0146182 1314

[22] M. Artin, On isolated rational singularities of surfaces, Amer. J. Math. 88 (1966), 129-136, DOI 10.2307/2373050. MR0199191 1314

[23] Y. Barreto, S. López de Medrano, and A. Verjovsky, Some open book and contact structures on moment-angle manifolds, Bol. Soc. Mat. Mex. (3) 23 (2017), no. 1, 423-437, DOI 10.1007/s40590-016-0113-y. MR3633142 1334

[24] K. Bekka, C-régularité et trivialité topologique (French), Singularity theory and its applications, Part I (Coventry, 1988/1989), Lecture Notes in Math., vol. 1462, Springer, Berlin, 1991, pp. 42-62, DOI 10.1007/BFb0086373. MR1129023 1323

[25] K. Behrend, Donaldson-Thomas type invariants via microlocal geometry, Ann. of Math. (2) 170 (2009), no. 3, 1307-1338, DOI 10.4007/annals.2009.170.1307. MR2600874 1306

[26] L. Birbrair, Local bi-Lipschitz classification of 2-dimensional semialgebraic sets, Houston J. Math. 25 (1999), no. 3, 453-472. MR.1730886 309

[27] L. Birbrair and A. Fernandes, Inner metric geometry of complex algebraic surfaces with isolated singularities, Comm. Pure Appl. Math. 61 (2008), no. 11, 1483-1494, DOI 10.1002/cpa.20244. MR2444373 1309

[28] L. Birbrair, A. Fernandes, D. T. Lê, and J. E. Sampaio, Lipschitz regular complex algebraic sets are smooth, Proc. Amer. Math. Soc. 144 (2016), no. 3, 983-987, DOI 10.1090/proc/12783. MR 3447652 309 310

[29] L. Birbrair, A. Fernandes, and W. D. Neumann, Bi-Lipschitz geometry of complex surface singularities, Geom. Dedicata 139 (2009), 259-267, DOI 10.1007/s10711-008-9333-2. MR2481850 309

[30] L. Birbrair and T. Mostowski, Normal embeddings of semialgebraic sets, Michigan Math. J. 47 (2000), no. 1, 125-132, DOI 10.1307/mmj/1030374672. MR 1755260 1309

[31] L. Birbrair, W. D. Neumann, and A. Pichon, The thick-thin decomposition and the bilipschitz classification of normal surface singularities, Acta Math. 212 (2014), no. 2, 199-256, DOI 10.1007/s11511-014-0111-8. MR3207758 1309

[32] B. Bode. Constructing links of isolated singularities of polynomials $\mathbb{R}^{4} \rightarrow \mathbb{R}^{2}$, arXiv:1705.09255 (2018). 328

[33] A. Bodin and A. Pichon, Meromorphic functions, bifurcation sets and fibred links, Math. Res. Lett. 14 (2007), no. 3, 413-422, DOI 10.4310/MRL.2007.v14.n3.a6. MR2318645 1284. 330

[34] A. Bodin, A. Pichon, and J. Seade, Milnor fibrations of meromorphic functions, J. Lond. Math. Soc. (2) 80 (2009), no. 2, 311-325, DOI 10.1112/jlms/jdp027. MR2545254 1284 330

[35] F. Bosio and L. Meersseman, Real quadrics in $\mathbf{C}^{n}$, complex manifolds and convex polytopes, Acta Math. 197 (2006), no. 1, 53-127, DOI 10.1007/s11511-006-0008-2. MR2285318 1334

[36] H.-Ch. Graf von Bothmer, W. Ebeling, and X. Gómez-Mont, An algebraic formula for the index of a vector field on an isolated complete intersection singularity (English, with English and French summaries), Ann. Inst. Fourier (Grenoble) 58 (2008), no. 5, 1761-1783. MR 2445833 1304

[37] J.-P. Brasselet and M.-H. Schwartz, Sur les classes de Chern d'un ensemble analytique complexe (French), The Euler-Poincaré characteristic (French), Astérisque, vol. 82, Soc. Math. France, Paris, 1981, pp. 93-147. MR629125 1305 306 
[38] J.-P. Brasselet, D. Lehmann, J. Seade, and T. Suwa, Milnor classes of local complete intersections, Trans. Amer. Math. Soc. 354 (2002), no. 4, 1351-1371, DOI 10.1090/S0002-994701-02846-X. MR 1873009 1306

[39] J.-P. Brasselet, Lê Dũng Tráng, and J. Seade, Euler obstruction and indices of vector fields, Topology 39 (2000), no. 6, 1193-1208, DOI 10.1016/S0040-9383(99)00009-9. MR.1783853 305

[40] J.-P. Brasselet, D. Massey, A. J. Parameswaran, and J. Seade, Euler obstruction and defects of functions on singular varieties, J. London Math. Soc. (2) 70 (2004), no. 1, 59-76, DOI 10.1112/S0024610704005447. MR2064752 1305

[41] J.-P. Brasselet, J. Seade, and T. Suwa, Vector fields on singular varieties, Lecture Notes in

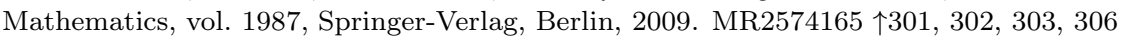

[42] J.-P. Brasselet, J. Schürmann, and S. Yokura, Hirzebruch classes and motivic Chern classes for singular spaces, J. Topol. Anal. 2 (2010), no. 1, 1-55, DOI 10.1142/S1793525310000239. MR2646988 306

[43] J.-P. Brasselet, J. Seade, and T. Suwa, Vector fields on singular varieties, Lecture Notes in Mathematics, vol. 1987, Springer-Verlag, Berlin, 2009. MR2574165 1301 $302 \quad 303 \quad 306$

[44] K. Brauner. Zur Geometrie der Funktionen zweier komplexen Veränderlichen III, IV. Abh. Math. Sem. Hamburg 6 (1928), 8-24. 311

[45] J. Briançon and J.-P. Speder, La trivialité topologique n'implique pas les conditions de Whitney (French, with English summary), C. R. Acad. Sci. Paris Sér. A-B 280 (1975), no. 6, Aiii, A365-A367. MR0425165 308

[46] J. Briançon and J.-P. Speder, Les conditions de Whitney impliquent " $\left({ }^{*}\right)$ constant" (French, with English summary), Ann. Inst. Fourier (Grenoble) 26 (1976), no. 2, xi, 153-163. MR0419825 308

[47] E. Brieskorn, Beispiele zur Differentialtopologie von Singularitäten (German), Invent. Math. 2 (1966), 1-14, DOI 10.1007/BF01403388. MR0206972 1282 286 287 312

[48] E. Brieskorn and H. Knörrer, Plane algebraic curves, Modern Birkhäuser Classics, Birkhäuser/Springer Basel AG, Basel, 1986. Translated from the German original by John Stillwell; [2012] reprint of the 1986 edition. MR2975988 1311

[49] B. Bories and W. Veys, Igusa's p-adic local zeta function and the monodromy conjecture for non-degenerate surface singularities, Mem. Amer. Math. Soc. 242 (2016), no. 1145, vii+131, DOI 10.1090/memo/1145. MR3498149 299

[50] Ch. Brücker and G.-M. Greuel, Deformationen isolierter Kurvensingluaritäten mit eingebetteten Komponenten, Manuscripta Math. 70 (1990), 93-114. 1301

[51] D. Burghelea and A. Verona, Local homological properties of analytic sets, Manuscripta Math. 7 (1972), 55-66, DOI 10.1007/BF01303536. MR0310285 1291

[52] R.-O. Buchweitz and G.-M. Greuel, The Milnor number and deformations of complex curve singularities, Invent. Math. 58 (1980), no. 3, 241-281, DOI 10.1007/BF01390254. MR571575 300.301

[53] R. Callejas-Bedregal, M. F. Z. Morgado, and J. Seade, Lê cycles and Milnor classes, Invent. Math. 197 (2014), no. 2, 453-482; erratum, 483-489, DOI 10.1007/s00222-013-0450-7. MR.3232012 1306

[54] R. Callejas-Bedregal, M. F. Z. Morgado, and J. Seade, On the total Milnor class of complete intersections, preprint (2018). 307

[55] R. Callejas-Bedregal, M. F. Z. Morgado. and J. Seade, On the Chern classes of singular complete intersections, preprint (2018). 306307

[56] R. Callejas-Bedregal, M. F. Z. Morgado, M. Saia, and J. Seade, The Lê-Greuel formula for functions on analytic spaces, Tohoku Math. J. (2) 68 (2016), no. 3, 439-456, DOI 10.2748/tmj/1474652267. MR 3550927 300

[57] C. Camacho, N. H. Kuiper, and J. Palis, The topology of holomorphic flows with singularity, Inst. Hautes Études Sci. Publ. Math. 48 (1978), 5-38. MR.516913 333333

[58] S. E. Cappell, L. Maxim, J. Schürmann, and J. L. Shaneson, Characteristic classes of complex hypersurfaces, Adv. Math. 225 (2010), no. 5, 2616-2647, DOI 10.1016/j.aim.2010.05.007. MR.2680178 306

[59] C. Caubel, A. Némethi, and P. Popescu-Pampu, Milnor open books and Milnor fillable contact 3-manifolds, Topology 45 (2006), no. 3, 673-689, DOI 10.1016/j.top.2006.01.002. MR2218761 312313 
[60] T. Cauwbergs and W. Veys, Monodromy eigenvalues and poles of zeta functions, Bull. Lond. Math. Soc. 49 (2017), no. 2, 342-350, DOI 10.1112/blms.12003. MR3656302 299

[61] P. T. Church and K. Lamotke, Non-trivial polynomial isolated singularities, Nederl. Akad. Wetensch. Proc. Ser. A 78=Indag. Math. 37 (1975), 149-154. MR0365592 1318

[62] J. L. Cisneros-Molina, Join theorem for polar weighted homogeneous singularities, Singularities II, Contemp. Math., vol. 475, Amer. Math. Soc., Providence, RI, 2008, pp. 43-59, DOI 10.1090/conm/475/09274. MR2454370 1284,327

[63] J. L. Cisneros-Molina, J. Seade, and N. G. Grulha Jr., On the topology of real analytic maps, Internat. J. Math. 25 (2014), no. 7, 1450069, 30, DOI 10.1142/S0129167X14500694. MR.3238091 $1300 \quad 322 \quad 323 \quad 326$

[64] J. L. Cisneros-Molina and A. Romano-Velázquez, Classification of isolated polar weighted homogeneous singularities, Singularities in geometry, topology, foliations and dynamics, Trends Math., Birkhäuser/Springer, Cham, 2017, pp. 77-104. MR3706214 328

[65] J. L. Cisneros-Molina, J. Seade, and J. Snoussi, Refinements of Milnor's fibration theorem for complex singularities, Adv. Math. 222 (2009), no. 3, 937-970, DOI 10.1016/j.aim.2009.05.010. MR2553374 1294 295 325

[66] J. L. Cisneros-Molina, J. Seade, and J. Snoussi, Milnor fibrations and d-regularity for real analytic singularities, Internat. J. Math. 21 (2010), no. 4, 419-434, DOI 10.1142/S0129167X10006124. MR2647448 1317 323 324 325

[67] J. L. Cisneros-Molina, J. Seade, and J. Snoussi, Milnor fibrations and the concept of d-regularity for analytic map germs, Real and complex singularities, Contemp. Math., vol. 569, Amer. Math. Soc., Providence, RI, 2012, pp. 1-28, DOI 10.1090/conm/569/11241. MR.2934587 321325

[68] J. L. Cisneros-Molina, A. Menegón, J. Seade, and J. Snoussi, Fibration theorems and $d$ regularity for differentiable map-germs with non-isolated critical value, preprint (2017). $321322,325,326$

[69] O. Collin and N. Saveliev, A geometric proof of the Fintushel-Stern formula, Adv. Math. 147 (1999), no. 2, 304-314, DOI 10.1006/aima.1999.1842. MR.1734525 1316

[70] R. de Souza Martins and A. Menegon, Milnor-Lê type fibrations for subanalytic maps, arXiv:1806.05349 (2018). 1316

[71] A. Dimca, Singularities and topology of hypersurfaces, Universitext, Springer-Verlag, New York, 1992. MR1194180 291 299

[72] I. V. Dolgačev, Conic quotient singularities of complex surfaces (Russian), Funkcional. Anal. i Priložen. 8 (1974), no. 2, 75-76. MR 0345974 1313 314

[73] I. V. Dolgačev, Automorphic forms, and quasihomogeneous singularities (Russian), Funkcional. Anal. i Priložen. 9 (1975), no. 2, 67-68. MR0568895 313314

[74] A. A. du Plessis and C. T. C. Wall, Discriminants, vector fields and singular hypersurfaces, New developments in singularity theory (Cambridge, 2000), NATO Sci. Ser. II Math. Phys. Chem., vol. 21, Kluwer Acad. Publ., Dordrecht, 2001, pp. 351-377. MR.1849316 293

[75] A. S. Dubson, Classes caractéristiques des variétés singulières (French, with English summary), C. R. Acad. Sci. Paris Sér. A-B 287 (1978), no. 4, A237-A240. MR0499290 1305

[76] A. H. Durfee, The signature of smoothings of complex surface singularities, Math. Ann. 232 (1978), no. 1, 85-98, DOI 10.1007/BF01420624. MR.0466620 1314 315

[77] A. H. Durfee, Neighborhoods of algebraic sets, Trans. Amer. Math. Soc. 276 (1983), no. 2, 517-530, DOI 10.2307/1999065. MR688959 288 291

[78] A. H. Durfee and H. B. Lawson Jr., Fibered knots and foliations of highly connected manifolds, Invent. Math. 17 (1972), 203-215, DOI 10.1007/BF01425448. MR0326752 1310

[79] N. Dutertre, On the topology of semi-algebraic functions on closed semi-algebraic sets, Manuscripta Math. 139 (2012), no. 3-4, 415-441, DOI 10.1007/s00229-011-0523-0. MR 2974284 316

[80] N. Dutertre, R. N. Araújo dos Santos, Y. Chen, and A. Andrade do Espirito Santo, Open book structures on semi-algebraic manifolds, Manuscripta Math. 149 (2016), no. 1-2, 205222, DOI 10.1007/s00229-015-0772-4. MR3447150 1316 320

[81] N. Dutertre and N. G. Grulha Jr., Lê-Greuel type formula for the Euler obstruction and applications, Adv. Math. 251 (2014), 127-146, DOI 10.1016/j.aim.2013.10.023. MR.3130338 1300,323

[82] N. Dutertre, R. Araújo dos Santos, Y. Chen, and A. Andrade do Espirito Santo, Fibration structures and formulae for the Euler characteristics of Milnor fibers, Theory of singularities 
of smooth mappings and around it, RIMS Kôkyûroku Bessatsu, B55, Res. Inst. Math. Sci. (RIMS), Kyoto, 2016, pp. 41-65. MR3617499 320

[83] W. Ebeling, Milnor lattices and geometric bases of some special singularities, Enseign. Math. (2) 29 (1983), no. 3-4, 263-280. MR719312 1299

[84] W. Ebeling, Monodromy, Singularities and computer algebra, London Math. Soc. Lecture Note Ser., vol. 324, Cambridge Univ. Press, Cambridge, 2006, pp. 129-155, DOI 10.1017/CBO9780511526374.008. MR2228229 1299

[85] W. Ebeling and S. M. Gusein-Zade, On the index of a vector field at an isolated singularity, The Arnoldfest (Toronto, ON, 1997), Fields Inst. Commun., vol. 24, Amer. Math. Soc., Providence, RI, 1999, pp. 141-152. MR1733572 1301 30232

[86] W. Ebeling and S. M. Gusein-Zade, Radial index and Euler obstruction of a 1-form on a singular variety, Geom. Dedicata 113 (2005), 231-241, DOI 10.1007/s10711-005-2184-1. MR2171307 301305

[87] W. Ebeling and S. M. Gusein-Zade, Indices of vector fields or 1-forms and characteristic numbers, Bull. London Math. Soc. 37 (2005), no. 5, 747-754, DOI 10.1112/S0024609305004789. MR2164837 301 305

[88] W. Ebeling, S. M. Gusein-Zade, and J. Seade, Homological index for 1-forms and a Milnor number for isolated singularities, Internat. J. Math. 15 (2004), no. 9, 895-905, DOI 10.1142/S0129167X04002624. MR2106152 1301

[89] D. Eisenbud and H. I. Levine, An algebraic formula for the degree of a $C^{\infty}$ map germ, Ann. of Math. (2) 106 (1977), no. 1, 19-44, DOI 10.2307/1971156. With an appendix by Bernard Teissier, "Sur une inégalité à la Minkowski pour les multiplicités". MR0467800 1323

[90] D. Eisenbud and W. Neumann, Three-dimensional link theory and invariants of plane curve singularities, Annals of Mathematics Studies, vol. 110, Princeton University Press, Princeton, NJ, 1985. MR817982 1329

[91] Y. Eliashberg, Filling by holomorphic discs and its applications, Geometry of lowdimensional manifolds, 2 (Durham, 1989), London Math. Soc. Lecture Note Ser., vol. 151, Cambridge Univ. Press, Cambridge, 1990, pp. 45-67. MR.1171908 313

[92] H. Esnault, J. Seade, and E. Viehweg, Characteristic divisors on complex manifolds, J. Reine Angew. Math. 424 (1992), 17-30. MR.1144159 1315

[93] C. Eyral, Zariski's multiplicity question-a survey, New Zealand J. Math. 36 (2007), 253276. MR 2476643 1308

[94] J. Fernández de Bobadilla, Answers to some equisingularity questions, Invent. Math. 161 (2005), no. 3, 657-675, DOI 10.1007/s00222-005-0441-4. MR2181471 1307 309

[95] J. Fernández de Bobadilla, Topological equisingularity of hypersurfaces with 1-dimensional critical set, Adv. Math. 248 (2013), 1199-1253, DOI 10.1016/j.aim.2013.08.001. MR.3107539 307309

[96] J. Fernández de Bobadilla, On homotopy types of complements of analytic sets and Milnor fibres, Topology of algebraic varieties and singularities, Contemp. Math., vol. 538, Amer. Math. Soc., Providence, RI, 2011, pp. 363-367, DOI 10.1090/conm/538/10612. MR 2777829 309

[97] J. Fernández de Bobadilla and A. Menegon Neto, The boundary of the Milnor fibre of complex and real analytic non-isolated singularities, Geom. Dedicata 173 (2014), 143-162, DOI 10.1007/s10711-013-9933-3. MR3275295 1316 321 330.

[98] A. Fernandes and J. E. Sampaio, Multiplicity of analytic hypersurface singularities under biLipschitz homeomorphisms, J. Topol. 9 (2016), no. 3, 927-933, DOI 10.1112/jtopol/jtw014. MR 3551843 308 309 310

[99] M. Freedman and R. Kirby, A geometric proof of Rochlin's theorem, Algebraic and geometric topology (Proc. Sympos. Pure Math., Stanford Univ., Stanford, Calif., 1976), Proc. Sympos. Pure Math., XXXII, Amer. Math. Soc., Providence, R.I., 1978, pp. 85-97. MR.520525 1315

[100] R. Fintushel and R. J. Stern, Instanton homology of Seifert fibred homology three spheres, Proc. London Math. Soc. (3) 61 (1990), no. 1, 109-137, DOI 10.1112/plms/s3-61.1.109. MR.1051101 315

[101] W. Fulton, Intersection theory, Ergebnisse der Mathematik und ihrer Grenzgebiete (3) [Results in Mathematics and Related Areas (3)], vol. 2, Springer-Verlag, Berlin, 1984. MR 732620 1305 306

[102] W. Fulton and K. Johnson, Canonical classes on singular varieties, Manuscripta Math. 32 (1980), no. 3-4, 381-389, DOI 10.1007/BF01299611. MR595428 306 307 
[103] A. M. Gabrièlov, Intersection matrices for certain singularities (Russian), Funkcional. Anal. i Priložen. 7 (1973), no. 3, 18-32. MR0324066 1299

[104] T. Gaffney, Integral closure of modules and Whitney equisingularity, Invent. Math. 107 (1992), no. 2, 301-322, DOI 10.1007/BF01231892. MR.1144426 1307 319

[105] T. Gaffney, Multiplicities and equisingularity of ICIS germs, Invent. Math. 123 (1996), no. 2, 209-220, DOI 10.1007/s002220050022. MR1374196 1307

[106] T. Gaffney, Non-isolated complete intersection singularities and the $A_{f}$ condition, Singularities I, Contemp. Math., vol. 474, Amer. Math. Soc., Providence, RI, 2008, pp. 85-93, DOI 10.1090/conm/474/09253. MR2454346 1307

[107] T. Gaffney, Bi-Lipschitz equivalence, integral closure and invariants, Real and complex singularities, London Math. Soc. Lecture Note Ser., vol. 380, Cambridge Univ. Press, Cambridge, 2010, pp. 125-137. MR2759079 1309

[108] T. Gaffney, Equisingularity and the theory of integral closure, Singularities and foliations. geometry, topology and applications, Springer Proc. Math. Stat., vol. 222, Springer, Cham, 2018, pp. 89-131. MR 3779559 309

[109] E. Giroux, Géométrie de contact: de la dimension trois vers les dimensions supérieures (French, with French summary), Proceedings of the International Congress of Mathematicians, Vol. II (Beijing, 2002), Higher Ed. Press, Beijing, 2002, pp. 405-414. MR1957051 1312

[110] S. Gitler and S. López de Medrano, Intersections of quadrics, moment-angle manifolds and connected sums, Geom. Topol. 17 (2013), no. 3, 1497-1534, DOI 10.2140/gt.2013.17.1497. MR 3073929 334

[111] X. Gómez-Mont, J. Seade, and A. Verjovsky, The index of a holomorphic flow with an isolated singularity, Math. Ann. 291 (1991), no. 4, 737-751, DOI 10.1007/BF01445237. MR 1135541 1303

[112] X. Gómez-Mont, An algebraic formula for the index of a vector field on a hypersurface with an isolated singularity, J. Algebraic Geom. 7 (1998), no. 4, 731-752. MR1642757 303

[113] X. Gómez-Mont and P. Mardešić, The index of a vector field tangent to a hypersurface and the signature of the relative Jacobian determinant (English, with English and French summaries), Ann. Inst. Fourier (Grenoble) 47 (1997), no. 5, 1523-1539. MR 1600363 1323

[114] X. Gomes-Mont and P. Mardešić, The index of a vector field tangent to an odd-dimensional hypersurface, and the signature of the relative Hessian (Russian, with Russian summary), Funktsional. Anal. i Prilozhen. 33 (1999), no. 1, 1-13, 96, DOI 10.1007/BF02465138; English transl., Funct. Anal. Appl. 33 (1999), no. 1, 1-10. MR.1711866 1323

[115] G. González-Sprinberg, L'obstruction locale d'Euler et le théorème de MacPherson (French), The Euler-Poincaré characteristic (French), Astérisque, vol. 83, Soc. Math. France, Paris, 1981, pp. 7-32. MR629121 305

[116] M. Goresky and R. MacPherson, Stratified Morse theory, Ergebnisse der Mathematik und ihrer Grenzgebiete (3) [Results in Mathematics and Related Areas (3)], vol. 14, SpringerVerlag, Berlin, 1988. MR932724 1291, 294

[117] G. M. Greuel, Equisingular and equinormalizable deformations of isolated non-normal singularities, Methods Appl. Anal. 24 (2017), 215-276. 301 307

[118] G.-M. Greuel, Der Gauss-Manin-Zusammenhang isolierter Singularitäten von vollständigen Durchschnitten, Math. Ann. 214 (1975), 235-266, DOI 10.1007/BF01352108. MR0396554 300,301

[119] G. M. Greuel and W. Purket, Life and work of Egbert Brieskorn (1936-2013), J. Singularities 18 (2018), 7-34; Special volume in honor of the life and mathematics of Egbert Brieskorn. arXiv:1711.09600 (2017). 1286

[120] G.-M. Greuel and E. Shustin, Geometry of equisingular families of curves, Singularity theory (Liverpool, 1996), London Math. Soc. Lecture Note Ser., vol. 263, Cambridge Univ. Press, Cambridge, 1999, pp. xvi, 79-108. MR 1709347 1294 309

[121] G.-M. Greuel and J. Steenbrink, On the topology of smoothable singularities, Singularities, Part 1 (Arcata, Calif., 1981), Proc. Sympos. Pure Math., vol. 40, Amer. Math. Soc., Providence, R.I., 1983, pp. 535-545. MR713090 1301

[122] G. M. Greuel, Ch. Lossen, and E. Shustin, Singular Algebraic Curves, Springer Verlag, 2018 (to appear). 311 
[123] A. Grothendieck. Récoltes et Semailles; Réflexions et Témoignages sur un passé de mathématicien, 1985, https://www.quarante-deux.org/archives/klein/prefaces/ Romans_1965-1969/Recoltes_et_semailles.pdf. 1306

[124] N. de Góes Grulha Júnior, L'obstruction d'Euler locale d'une application (French, with English and French summaries), Ann. Fac. Sci. Toulouse Math. (6) 17 (2008), no. 1, 53-71. MR2464093 305

[125] S. M. Gusein-Zade, I. Luengo, and A. Melle-Hernández, Zeta functions of germs of meromorphic functions, and the Newton diagram (Russian, with Russian summary), Funktsional. Anal. i Prilozhen. 32 (1998), no. 2, 26-35, 95, DOI 10.1007/BF02482595; English transl., Funct. Anal. Appl. 32 (1998), no. 2, 93-99. MR 1647824 1284 299,330

[126] S. M. Guseřn-Zade, I. Luengo, and A. Melle-Hernández, On the topology of germs of meromorphic functions and its applications (Russian), Algebra i Analiz 11 (1999), no. 5, 92-99; English transl., St. Petersburg Math. J. 11 (2000), no. 5, 775-780. MR.1734347 284 330

[127] A. Haefliger, Deformations of transversely holomorphic flows on spheres and deformations of Hopf manifolds, Compositio Math. 55 (1985), no. 2, 241-251. MR795716 1334

[128] H. Hamm, Lokale topologische Eigenschaften komplexer Räume (German), Math. Ann. 191 (1971), 235-252, DOI 10.1007/BF01578709. MR0286143 1287 $2 9 3 \longdiv { 2 9 9 }$

[129] H. A. Hamm and Lê Dũng Tráng, Un théorème de Zariski du type de Lefschetz, Ann. Sci. École Norm. Sup. (4) 6 (1973), 317-355. MR0401755 297 320 321

[130] M. Hirasawa and L. Rudolph, Constructions of Morse maps for knots and links, and upper bounds on the Morse-Novikov number, arXiv:math/0311134 (2003). 1284

[131] H. Hironaka, Stratification and flatness, Real and complex singularities (Proc. Ninth Nordic Summer School/NAVF Sympos. Math., Oslo, 1976), Sijthoff and Noordhoff, Alphen aan den Rijn, 1977, pp. 199-265. MR0499286 292

[132] H. Hironaka, Subanalytic sets, Number theory, algebraic geometry and commutative algebra, in honor of Yasuo Akizuki, Kinokuniya, Tokyo, 1973, pp. 453-493. MR0377101 308

[133] F. Hirzebruch, Topological methods in algebraic geometry, Third enlarged edition. New appendix and translation from the second German edition by R. L. E. Schwarzenberger, with an additional section by A. Borel. Die Grundlehren der Mathematischen Wissenschaften, Band 131, Springer-Verlag New York, Inc., New York, 1966. MR0202713 1285

[134] F. Hirzebruch, Singularities and exotic spheres, Séminaire Bourbaki, Vol. 10, Soc. Math. France, Paris, 1995, pp. Exp. No. 314, 13-32. MR1610436 1286 287

[135] K. Inaba, On the enhancement to the Milnor number of a class of mixed polynomials, J. Math. Soc. Japan 66 (2014), no. 1, 25-36, DOI 10.2969/jmsj/06610025. MR3161391 1328

[136] K. Inaba, On deformations of isolated singularities of polar weighted homogeneous mixed polynomials, Osaka J. Math. 53 (2016), no. 3, 813-842. MR3533471 1328

[137] K. Inaba, Topology of the Milnor fibrations of polar weighted homogeneous polynomials, Manuscripta Math. 157 (2018), no. 3-4, 411-424, DOI 10.1007/s00229-018-0998-z. MR.3858409 328

[138] A. Jacquemard, Fibrations de Milnor pour des applications réelles (French, with Italian summary), Boll. Un. Mat. Ital. B (7) 3 (1989), no. 3, 591-600. MR1010525 1319

[139] D. Kerner, H. M. Pedersen, and M. A. S. Ruas, Lipschitz normal embeddings in the space of matrices, Math. Z. 290 (2018), no. 1-2, 485-507, DOI 10.1007/s00209-017-2027-4. MR.3848442 309

[140] M. A. Kervaire and J. W. Milnor, Groups of homotopy spheres. I, Ann. of Math. (2) 77 (1963), 504-537, DOI 10.2307/1970128. MR0148075 1285 286

[141] H. King and D. Trotman, Poincaré-Hopf theorems on singular spaces, Proc. Lond. Math. Soc. (3) 108 (2014), no. 3, 682-703, DOI 10.1112/plms/pdt039. MR3180593 1302

[142] R. C. Kirby, The topology of 4-manifolds, Lecture Notes in Mathematics, vol. 1374, SpringerVerlag, Berlin, 1989. MR1001966 1315

[143] F. Klein, Lectures on the icosahedron and the solution of equations of the fifth degree, Second and revised edition, Dover Publications, Inc., New York, N.Y., 1956. Translated into English by George Gavin Morrice. MR.0080930 1313

[144] F. Larrión and J. Seade, Complex surface singularities from the combinatorial point of view, Topology Appl. 66 (1995), no. 3, 251-265, DOI 10.1016/0166-8641(95)00038-I. MR.1359516 1315 
[145] T. László and A. Némethi, Ehrhart theory of polytopes and Seiberg-Witten invariants of plumbed 3-manifolds, Geom. Topol. 18 (2014), no. 2, 717-778, DOI 10.2140/gt.2014.18.717. MR.3180484 1316

[146] H. B. Laufer, On $\mu$ for surface singularities, Several complex variables (Proc. Sympos. Pure Math., Vol. XXX, Part 1, Williams Coll., Williamstown, Mass., 1975), Amer. Math. Soc., Providence, R. I., 1977, pp. 45-49. MR0450287 1301 314

[147] Lê Dũng Tráng, Deux nœuds algébriques FM-équivalents sont égaux (French), C. R. Acad. Sci. Paris Sér. A-B 272 (1971), A214-A216. MR0281190个

[148] Lê Dũng Tráng, Sur un critère d'équisingularité (French), C. R. Acad. Sci. Paris Sér. A-B 272 (1971), A138-A140. MR0273649 个

[149] Lê Dũng Tráng, Calcul du nombre de cycles évanouissants d'une hypersurface complexe (French, with English summary), Ann. Inst. Fourier (Grenoble) 23 (1973), no. 4, 261-270. MR.0330501 297

[150] Lê Dũng Tráng, Computation of the Milnor number of an isolated singularity of a complete intersection (Russian), Funkcional. Anal. i Priložen. 8 (1974), no. 2, 45-49. MR0350064 1300

[151] Lê Dũng Tráng, Un critère d'équisingularité (French), Singularités à Cargèse (Rencontre Singularités Géom. Anal., Inst. Études Sci., Cargèse, 1972), Soc. Math. France, Paris, 1973, pp. 183-192. Astérisque, Nos. 7 et 8. MR0361148 307

[152] Lê Dũng Tráng, Topological use of polar curves, Algebraic geometry (Proc. Sympos. Pure Math., Vol. 29, Humboldt State Univ., Arcata, Calif., 1974), Amer. Math. Soc., Providence, R.I., 1975, pp. 507-512. MR0374481 297

[153] Lé Dũng Tráng, Vanishing cycles on complex analytic sets, Sûrikaisekikenkyûsho Kókyûroku 266 (1976), 299-318. Various problems in algebraic analysis (Proc. Sympos., Res. Inst. Math. Sci., Kyoto Univ., Kyoto, 1975). MR0477132 1297

[154] Lê Dũng Tráng, Some remarks on relative monodromy, Real and complex singularities (Proc. Ninth Nordic Summer School/NAVF Sympos. Math., Oslo, 1976), Sijthoff and Noordhoff, Alphen aan den Rijn, 1977, pp. 397-403. MR0476739 1294 310

[155] Lê Dũng Tráng, Polyèdres évanescents et effondrements (French), A fête of topology, Academic Press, Boston, MA, 1988, pp. 293-329. MR928405 1296

[156] Lê Dũng Tráng and C. P. Ramanujam, The invariance of Milnor's number implies the invariance of the topological type, Amer. J. Math. 98 (1976), no. 1, 67-78, DOI 10.2307/2373614. MR0399088 307 308

[157] Lê Dũng Tráng and B. Perron, Sur la fibre de Milnor d'une singularité isolée en dimension complexe trois (French, with English summary), C. R. Acad. Sci. Paris Sér. A-B 289 (1979), no. 2, A115-A118. MR549082 $1 2 9 6 \longdiv { 2 9 7 }$

[158] Lê Dũng Tráng and B. Teissier, Variétés polaires locales et classes de Chern des variétés singulières (French), Ann. of Math. (2) 114 (1981), no. 3, 457-491, DOI 10.2307/1971299. MR634426 1305

[159] Lê Dũng Tráng and B. Teissier, Cycles evanescents, sections planes et conditions de Whitney. II (French, with English summary), Singularities, Part 2 (Arcata, Calif., 1981), Proc. Sympos. Pure Math., vol. 40, Amer. Math. Soc., Providence, RI, 1983, pp. 65-103. MR713238 1291

[160] D. T. Lê and D. B. Massey, Hypersurface singularities and Milnor equisingularity, Pure Appl. Math. Q. 2 (2006), no. 3, Special Issue: In honor of Robert D. MacPherson., 893-914, DOI 10.4310/PAMQ.2006.v2.n3.a13. MR2252122 1307 308

[161] L. D. Tráng and A. M. Neto, Vanishing polyhedron and collapsing map, Math. Z. 286 (2017), no. 3-4, 1003-1040, DOI 10.1007/s00209-016-1793-8. MR3671569 1296

[162] D. Lehmann, M. Soares, and T. Suwa, On the index of a holomorphic vector field tangent to a singular variety, Bol. Soc. Brasil. Mat. (N.S.) 26 (1995), no. 2, 183-199, DOI 10.1007/BF01236993. MR.1364267 1303

[163] Y. Lim, The equivalence of Seiberg-Witten and Casson invariants for homology 3-spheres, Math. Res. Lett. 6 (1999), no. 5-6, 631-643, DOI 10.4310/MRL.1999.v6.n6.a4. MR1739221 1316

[164] Y. Lim, A non-abelian Seiberg-Witten invariant for integral homology 3-spheres, Geom. Topol. 7 (2003), 965-999, DOI 10.2140/gt.2003.7.965. MR2026552 $\uparrow$ 
[165] J. Lipman, Equisingularity and simultaneous resolution of singularities, Resolution of singularities (Obergurgl, 1997), Progr. Math., vol. 181, Birkhäuser, Basel, 2000, pp. 485-505, DOI 10.1007/978-3-0348-8399-3_17. MR.1748631 1309

[166] E. Looijenga, A note on polynomial isolated singularities, Nederl. Akad. Wetensch. Proc. Ser. A 74=Indag. Math. 33 (1971), 418-421. MR0303557 318

[167] E. J. N. Looijenga, Isolated singular points on complete intersections, London Mathematical Society Lecture Note Series, vol. 77, Cambridge University Press, Cambridge, 1984.

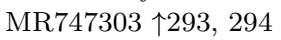

[168] S. Lojasiewicz, Triangulation of semi-analytic sets, Ann. Scuola Norm. Sup. Pisa (3) 18 (1964), 449-474. MR0173265 290

[169] S. López de Medrano, The space of Siegel leaves of a holomorphic vector field, Holomorphic dynamics (Mexico, 1986), Lecture Notes in Math., vol. 1345, Springer, Berlin, 1988, pp. 233245, DOI 10.1007/BFb0081404. MR980961 1332 334

[170] S. López de Medrano, Topology of the intersection of quadrics in $\mathbf{R}^{n}$, Algebraic topology (Arcata, CA, 1986), Lecture Notes in Math., vol. 1370, Springer, Berlin, 1989, pp. 280-292, DOI 10.1007/BFb0085235. MR1000384 334

[171] S. López de Medrano, Singularities of homogeneous quadratic mappings, Rev. R. Acad. Cienc. Exactas Fís. Nat. Ser. A Math. RACSAM 108 (2014), no. 1, 95-112, DOI 10.1007/s13398-012-0102-6. MR3183109 322 334

[172] S. López de Medrano, Singular intersections of quadrics I, Singularities in geometry, topology, foliations and dynamics, Trends Math., Birkhäuser/Springer, Cham, 2017, pp. 155-170. MR 3706218 334

[173] S. López de Medrano and A. Verjovsky, A new family of complex, compact, non-symplectic manifolds, Bol. Soc. Brasil. Mat. (N.S.) 28 (1997), no. 2, 253-269, DOI 10.1007/BF01233394. MR 1479504 1334

[174] R. D. MacPherson, Chern classes for singular algebraic varieties, Ann. of Math. (2) 100 (1974), 423-432, DOI 10.2307/1971080. MR0361141 304 305306

[175] D. B. Massey, The Lê varieties. I, Invent. Math. 99 (1990), no. 2, 357-376, DOI 10.1007/BF01234423. MR 1031905 283, 298, 309

[176] D. B. Massey, The Lê varieties. II, Invent. Math. 104 (1991), no. 1, 113-148, DOI 10.1007/BF01245068. MR 1094048 1283, 298, 307, 309

[177] D. B. Massey, Lê cycles and hypersurface singularities, Lecture Notes in Mathematics, vol. 1615, Springer-Verlag, Berlin, 1995. MR 1441075 295 298, 309

[178] D. B. Massey, Milnor fibres of non-isolated hypersurface singularities, Singularity theory (Trieste, 1991), World Sci. Publ., River Edge, NJ, 1995, pp. 458-467. MR1378416 1295

[179] D. B. Massey, An introduction to the Lê cycles of a hypersurface singularity, Singularity theory (Trieste, 1991), World Sci. Publ., River Edge, NJ, 1995, pp. 468-486, DOI 10.1007/BFb0094409. MR1378417 295,298

[180] D. B. Massey, Real analytic Milnor fibrations and a strong Eojasiewicz inequality, Real and complex singularities, London Math. Soc. Lecture Note Ser., vol. 380, Cambridge Univ. Press, Cambridge, 2010, pp. 268-292. MR2759069 1320 321

[181] J. N. Mather, Stratifications and mappings, Dynamical systems (Proc. Sympos., Univ. Bahia, Salvador, 1971), Academic Press, New York, 1973, pp. 195-232. MR.0368064 1295. 308

[182] L. Maxim, On Milnor classes of complex hypersurfaces, Topology of stratified spaces, Math. Sci. Res. Inst. Publ., vol. 58, Cambridge Univ. Press, Cambridge, 2011, pp. 161175. MR2796411 306

[183] L. Maxim, M. Saito, and J. Schürmann, Hirzebruch-Milnor classes of complete intersections, Adv. Math. 241 (2013), 220-245, DOI 10.1016/j.aim.2013.04.001. MR.3053711 1306

[184] L. Meersseman, A new geometric construction of compact complex manifolds in any dimension, Math. Ann. 317 (2000), no. 1, 79-115, DOI 10.1007/s002080050360. MR1760670 1334

[185] L. Meersseman and A. Verjovsky, Holomorphic principal bundles over projective toric varieties, J. Reine Angew. Math. 572 (2004), 57-96, DOI 10.1515/crll.2004.054. MR2076120 1334

[186] L. Meersseman and A. Verjovsky, Sur les variétés $L V-M$ (French, with French summary), Singularities II, Contemp. Math., vol. 475, Amer. Math. Soc., Providence, RI, 2008, pp. 111134, DOI 10.1090/conm/475/09279. MR2454363 1334 
[187] A. Menegon Neto and J. Seade, On the Lê-Milnor fibration for real analytic maps, Math. Nachr. 290 (2017), no. 2-3, 382-392, DOI 10.1002/mana.201600066. MR.3607111 «323

[188] A. Menegon Neto and J. F. de Bobadilla, The boundary of the Milnor fibre of complex and real analytic non-isolated singularities, Geom. Dedicata 173 (2014), 143-162, DOI 10.1007/s10711-013-9933-3. MR3275295 321 330

[189] F. Michel and A. Pichon, On the boundary of the Milnor fibre of nonisolated singularities, Int. Math. Res. Not. 43 (2003), 2305-2311; erratum, Int. Math. Res. Not. 2004, no. 6, 309-310, DOI 10.1155/S107379280313098X. MR1998468 1316 330

[190] F. Michel and A. Pichon, Carrousel in family and non-isolated hypersurface singularities in $\mathbb{C}^{3}$, J. Reine Angew. Math. 720 (2016), 1-32, DOI 10.1515/crelle-2014-0074. MR3565967 1316 330

[191] J. Milnor, On manifolds homeomorphic to the 7-sphere, Ann. of Math. (2) 64 (1956), 399405, DOI 10.2307/1969983. MR0082103 1285

[192] J. W. Milnor. On isolated singularities of hypersurfaces, preprint June 1966. Unpublished. $284,291,292,316$

[193] J. Milnor, Singular points of complex hypersurfaces, Annals of Mathematics Studies, No. 61, Princeton University Press, Princeton, N.J.; University of Tokyo Press, Tokyo, 1968.

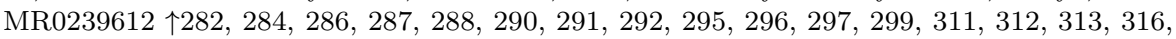
317318320,327

[194] J. Milnor, On the 3-dimensional Brieskorn manifolds $M(p, q, r)$, Knots, groups, and 3manifolds (Papers dedicated to the memory of R. H. Fox), Princeton Univ. Press, Princeton, N. J., 1975, pp. 175-225. Ann. of Math. Studies, No. 84. MR0418127 313,314

[195] D. Mond and D. van Straten, Milnor number equals Tjurina number for functions on space curves, J. London Math. Soc. (2) 63 (2001), no. 1, 177-187, DOI 10.1112/S0024610700001320. MR.1802765 301

[196] T. Mostowski, Lipschitz equisingularity, Dissertationes Math. (Rozprawy Mat.) 243 (1985), 46. MR808226 309

[197] T. Mostowski, A criterion for Lipschitz equisingularity (English, with Russian summary), Bull. Polish Acad. Sci. Math. 37 (1989), no. 1-6, 109-116 (1990). MR.1101458 1308 309

[198] T. Mrowka, P. Ozsváth, and B. Yu, Seiberg-Witten monopoles on Seifert fibered spaces, Comm. Anal. Geom. 5 (1997), no. 4, 685-791, DOI 10.4310/CAG.1997.v5.n4.a3. MR.1611061 1316

[199] D. Mumford, The topology of normal singularities of an algebraic surface and a criterion for simplicity, Inst. Hautes Études Sci. Publ. Math. 9 (1961), 5-22. MR0153682 1286 313

[200] A. Némethi, Invariants of normal surface singularities, Real and complex singularities, Contemp. Math., vol. 354, Amer. Math. Soc., Providence, RI, 2004, pp. 161-208, DOI 10.1090/conm/354/06481. MR2087811 1315

[201] A. Némethi, The Seiberg-Witten invariants of negative definite plumbed 3-manifolds, J. Eur. Math. Soc. (JEMS) 13 (2011), no. 4, 959-974, DOI 10.4171/JEMS/272. MR2800481 315,316

[202] A. Némethi and L. I. Nicolaescu, Seiberg-Witten invariants and surface singularities, Geom. Topol. 6 (2002), 269-328, DOI 10.2140/gt.2002.6.269. MR.1914570 314 315 316

[203] A. Némethi and L. I. Nicolaescu, Seiberg-Witten invariants and surface singularities. II. Singularities with good $\mathbb{C}^{*}$-action, J. London Math. Soc. (2) 69 (2004), no. 3, 593-607, DOI 10.1112/S0024610704005228. MR2050035 1314, 315 316

[204] A. Némethi and P. Popescu-Pampu, On the Milnor fibres of cyclic quotient singularities, Proc. Lond. Math. Soc. (3) 101 (2010), no. 2, 554-588, DOI 10.1112/plms/pdq007. MR2679701 313

[205] A. Némethi and Á. Szilárd, Milnor fiber boundary of a non-isolated surface singularity, Lecture Notes in Mathematics, vol. 2037, Springer, Heidelberg, 2012. MR 3024944 1316 330

[206] A. Némethi and B. Sigurdsson, The geometric genus of hypersurface singularities, J. Eur. Math. Soc. (JEMS) 18 (2016), no. 4, 825-851, DOI 10.4171/JEMS/604. MR3474458 1315. 316

[207] W. D. Neumann, A calculus for plumbing applied to the topology of complex surface singularities and degenerating complex curves, Trans. Amer. Math. Soc. 268 (1981), no. 2, 299-344, DOI 10.2307/1999331. MR632532 1313 314 
[208] W. D. Neumann, Geometry of quasihomogeneous surface singularities, Singularities, Part 2 (Arcata, Calif., 1981), Proc. Sympos. Pure Math., vol. 40, Amer. Math. Soc., Providence, RI, 1983, pp. 245-258. MR713253 1313 314

[209] W. Neumann and J. Wahl, Casson invariant of links of singularities, Comment. Math. Helv. 65 (1990), no. 1, 58-78, DOI 10.1007/BF02566593. MR1036128 1316

[210] W. D. Neumann and A. Pichon, Lipschitz geometry of complex curves, J. Singul. 10 (2014), 225-234. MR3300297 1309

[211] W. D. Neumann and A. Pichon, Lipschitz geometry does not determine embedded topological type, Singularities in geometry, topology, foliations and dynamics, Trends Math., Birkhäuser/Springer, Cham, 2017, pp. 183-195. MR3706220 1309

[212] W. D. Neumann and A. Pichon, Lipschitz geometry of complex surfaces: analytic invariants and equisingularity. arXiv:1211.4897 (2014). 309

[213] W. D. Neumann, H. M. Pedersen, and A. Pichon, A characterization of Lipschitz normally embedded surface singularities. arXiv:1806.11240 (2018). 1309

[214] W. D. Neumann, H. M. Pedersen, A. Pichon, Minimal singularities are Lisschitz normally embedded, arXiv:1503:03301 (2018). 1309

[215] N. Nguyen and G. Valette, Lipschitz stratifications in o-minimal structures (English, with English and French summaries), Ann. Sci. Éc. Norm. Supér. (4) 49 (2016), no. 2, 399-421, DOI 10.24033/asens.2286. MR3481354 1309

[216] J. J. Nuño-Ballesteros, B. Oréfice-Okamoto, and J. N. Tomazella, Equisingularity of families of isolated determinantal singularities, Math. Z. 289 (2018), no. 3-4, 1409-1425, DOI 10.1007/s00209-017-2004-y. MR3830255 307

[217] T. Ohmoto and S. Yokura, Product formulas for the Milnor class, Bull. Polish Acad. Sci. Math. 48 (2000), no. 4, 387-401. MR.1797904 306

[218] M. Oka, Principal zeta-function of nondegenerate complete intersection singularity, J. Fac. Sci. Univ. Tokyo Sect. IA Math. 37 (1990), no. 1, 11-32. MR.1049017 [299

[219] M. Oka, Non-degenerate complete intersection singularity, Actualités Mathématiques. [Current Mathematical Topics], Hermann, Paris, 1997. MR 1483897 284332

[220] M. Oka, Topology of polar weighted homogeneous hypersurfaces, Kodai Math. J. 31 (2008), no. 2, 163-182, DOI 10.2996/kmj/1214442793. MR2435890 320 328 332

[221] M. Oka, Non-degenerate mixed functions, Kodai Math. J. 33 (2010), no. 1, 1-62, DOI 10.2996/kmj/1270559157. MR2732230 320, 324 327 331 332

[222] M. Oka, On mixed Brieskorn variety, Topology of algebraic varieties and singularities, Contemp. Math., vol. 538, Amer. Math. Soc., Providence, RI, 2011, pp. 389-399, DOI 10.1090/conm/538/10615. MR2777832 284, 320 327 332

[223] M. Oka, On Milnor fibrations of mixed functions, $a_{f}$-condition and boundary stability, Kodai Math. J. 38 (2015), no. 3, 581-603, DOI 10.2996/kmj/1446210596. MR3417523 321 330. 332

[224] M. Oka, On the connectivity of Milnor fiber for mixed functions, arXiv:1809.00545 (2018). 320,321

[225] P. Orlik and P. Wagreich, Isolated singularities of algebraic surfaces with $C^{*}$ action, Ann. of Math. (2) 93 (1971), 205-228, DOI 10.2307/1970772. MR0284435

[226] A. J. Parameswaran, Topological equisingularity for isolated complete intersection singularities, Compositio Math. 80 (1991), no. 3, 323-336. MR 1134259

[227] A. Parusiński, A generalization of the Milnor number, Math. Ann. 281 (1988), no. 2, 247254, DOI 10.1007/BF01458431. MR949831 1300

[228] A. Parusiński, Lipschitz stratification, Global analysis in modern mathematics (Orono, ME, 1991; Waltham, MA, 1992) (K. Uhlenbeck, ed.), Publish or Perish, Houston, TX, 1993, pp. 73-89. MR 1278751 308 309

[229] A. Parusiński, Lipschitz properties of semi-analytic sets (English, with French summary), Ann. Inst. Fourier (Grenoble) 38 (1988), no. 4, 189-213. MR.978246 1309

[230] A. Parusiński and P. Pragacz, Characteristic classes of hypersurfaces and characteristic cycles, J. Algebraic Geom. 10 (2001), no. 1, 63-79. MR 1795550 306

[231] B. Perron, Le nøud "huit" est algébrique réel (French), Invent. Math. 65 (1981/82), no. 3, 441-451, DOI 10.1007/BF01396628. MR643562 1310

[232] F. Pham, Formules de Picard-Lefschetz généralisées et ramification des intégrales (French), Bull. Soc. Math. France 93 (1965), 333-367. MR0195868 1286 287 296 
[233] F. Pham, Fractions lipschitziennes et saturation de Zariski des algèbres analytiques complexes (French), Actes du Congrès International des Mathématiciens (Nice, 1970), GauthierVillars, Paris, 1971, pp. 649-654. Exposé d'un travail fait avec Bernard Teisser. Fractions lipschitziennes d'un algèbre analytique complexe et saturation de Zariski, Centre Math. École Polytech., Paris, 1969. MR0590058 \$309

[234] A. Pichon, Fibrations sur le cercle et surfaces complexes (French, with English summary), Ann. Inst. Fourier (Grenoble) 51 (2001), no. 2, 337-374. MR1824957 1311

[235] A. Pichon, Real analytic germs $f \bar{g}$ and open-book decompositions of the 3-sphere, Internat. J. Math. 16 (2005), no. 1, 1-12, DOI 10.1142/S0129167X05002710. MR2115674 1284 311 329,330

[236] A. Pichon and J. Seade, Real singularities and open-book decompositions of the 3-sphere (English, with English and French summaries), Ann. Fac. Sci. Toulouse Math. (6) 12 (2003), no. 2, 245-265. MR 2123256 284 320

[237] A. Pichon and J. Seade, Fibred multilinks and singularities f $\bar{g}$, Math. Ann. 342 (2008), no. 3, 487-514, DOI 10.1007/s00208-008-0234-3. MR2430986 284 311 317 320 321 324. 329330

[238] A. Pichon and J. Seade, Milnor fibrations and the Thom property for maps f $\bar{g}$, J. Singul. 3 (2011), 144-150; erratum, J. Singul. 7 (2013), 21-22. MR2861693 1330

[239] P. Popescu-Pampu, Complex singularities and contact topology, Winter Braids Lect. Notes 3 (2016), no. Winter Braids VI (Lille, 2016), Exp. No. 3, 74, DOI 10.5802/wbln.14. MR 3707744 311 312313

[240] A. Ranicki, High-dimensional knot theory, Springer Monographs in Mathematics, SpringerVerlag, New York, 1998. Algebraic surgery in codimension 2; With an appendix by Elmar Winkelnkemper. MR 1713074 310

[241] M. A. S. Ruas, J. Seade, and A. Verjovsky, On real singularities with a Milnor fibration, Trends in singularities, Trends Math., Birkhäuser, Basel, 2002, pp. 191-213. MR1900787 1284, 318, 319, 320, 323, 327

[242] L. Rudolph, Isolated critical points of mappings from $\mathbf{R}^{4}$ to $\mathbf{R}^{2}$ and a natural splitting of the Milnor number of a classical fibered link. I. Basic theory; examples, Comment. Math. Helv. 62 (1987), no. 4, 630-645, DOI 10.1007/BF02564467. MR920062 1327

[243] K. Saito, Calcul algébrique de la monodromie (French), Singularités à Cargèse (Rencontre Singularités Géom. Anal. Inst. Études Sci., Cargèse, 1972), Soc. Math. France, Paris, 1973, pp. 195-211. Astérisque, Nos. 7 et 8. MR0376670 1299

[244] M.-H. Schwartz, Classes caractéristiques définies par une stratification d'une variété analytique complexe. I (French), C. R. Acad. Sci. Paris 260 (1965), 3262-3264. MR0212842 302305

[245] J. Schürmann, Topology of singular spaces and constructible sheaves, Instytut Matematyczny Polskiej Akademii Nauk. Monografie Matematyczne (New Series) [Mathematics Institute of the Polish Academy of Sciences. Mathematical Monographs (New Series)], vol. 63, Birkhäuser Verlag, Basel, 2003. MR2031639 1306

[246] J. Schürmann, Chern classes and transversality for singular spaces. arXiv:1510.01986v3 (2016). 1307

[247] J. A. Séade, A cobordism invariant for surface singularities, Singularities, Part 2 (Arcata, Calif., 1981), Proc. Sympos. Pure Math., vol. 40, Amer. Math. Soc., Providence, R.I., 1983, pp. 479-484. MR.713273 315

[248] J. Seade, Open book decompositions associated to holomorphic vector fields, Bol. Soc. Mat. Mexicana (3) 3 (1997), no. 2, 323-335. MR 1679312, 1284, 320, 323] 327] 332

[249] J. Seade, On the topology of isolated singularities in analytic spaces, Progress in Mathematics, vol. 241, Birkhäuser Verlag, Basel, 2006. MR 2186327 1284 292 $313,314,327$

[250] J. Seade, Remarks on Laufer's formula for the Milnor number, Rochlin's signature theorem and the analytic Euler characteristic of compact complex manifolds, Methods Appl. Anal. 24 (2017), no. 1, 105-123, DOI 10.4310/MAA.2017.v24.n1.a8. MR3694303 1314 315

[251] J. Seade and T. Suwa, An adjunction formula for local complete intersections, Internat. J. Math. 9 (1998), no. 6, 759-768, DOI 10.1142/S0129167X98000324. MR1644307 303 306

[252] J. Seade, M. Tibăr, and A. Verjovsky, Milnor numbers and Euler obstruction, Bull. Braz. Math. Soc. (N.S.) 36 (2005), no. 2, 275-283, DOI 10.1007/s00574-005-0039-x. MR2152019 1305 
[253] M. Sebastiani and R. Thom, Un résultat sur la monodromie (French), Invent. Math. 13 (1971), 90-96, DOI 10.1007/BF01390095. MR0293122 1299

[254] L. Siebenmann and D. Sullivan, On complexes that are Lipschitz manifolds, Geometric topology (Proc. Georgia Topology Conf., Athens, Ga., 1977), Academic Press, New YorkLondon, 1979, pp. 503-525. MR.537747 1309

[255] D. Siersma, A bouquet theorem for the Milnor fibre, J. Algebraic Geom. 4 (1995), no. 1, 51-66. MR 1299004 1298

[256] S. Smale, On the structure of manifolds, Amer. J. Math. 84 (1962), 387-399, DOI 10.2307/2372978. MR0153022 1287

[257] J. H. M. Steenbrink, Mixed Hodge structures associated with isolated singularities, Singularities, Part 2 (Arcata, Calif., 1981), Proc. Sympos. Pure Math., vol. 40, Amer. Math. Soc., Providence, RI, 1983, pp. 513-536. MR713277 301 314

[258] D. Sullivan, Combinatorial invariants of analytic spaces, Proceedings of Liverpool Singularities-Symposium, I (1969/70), Springer, Berlin, 1971, pp. 165-168. MR0278333 1306

[259] D. Sullivan, On the intersection ring of compact three manifolds, Topology 14 (1975), no. 3, 275-277, DOI 10.1016/0040-9383(75)90009-9. MR0383415 1334

[260] D. Sullivan, Hyperbolic geometry and homeomorphisms, Geometric topology (Proc. Georgia Topology Conf., Athens, Ga., 1977), Academic Press, New York-London, 1979, pp. 543-555. MR.537749 1309

[261] T. Suwa, Classes de Chern des intersections complètes locales (French, with English and French summaries), C. R. Acad. Sci. Paris Sér. I Math. 324 (1997), no. 1, 67-70, DOI 10.1016/S0764-4442(97)80105-X. MR.1435589 306

[262] C. H. Taubes, Casson's invariant and gauge theory, J. Differential Geom. 31 (1990), no. 2, 547-599. MR1037415 315

[263] B. Teissier, Cycles évanescents, sections planes et conditions de Whitney (French), Singularités à Cargèse (Rencontre Singularités Géom. Anal., Inst. Études Sci., Cargèse, 1972), Soc. Math. France, Paris, 1973, pp. 285-362. Astérisque, Nos. 7 et 8. MR0374482 297 300. 307, 308 319

[264] B. Teissier, Introduction to equisingularity problems, Algebraic geometry (Proc. Sympos. Pure Math., Vol. 29, Humboldt State Univ., Arcata, Calif., 1974), Amer. Math. Soc., Providence, R.I., 1975, pp. 593-632. MR0422256 1307 308

[265] B. Teissier, Variétés polaires. I. Invariants polaires des singularités d'hypersurfaces (French), Invent. Math. 40 (1977), no. 3, 267-292, DOI 10.1007/BF01425742. MR0470246 297

[266] B. Teissier, Variétés polaires. II. Multiplicités polaires, sections planes, et conditions de Whitney (French), Algebraic geometry (La Rábida, 1981), Lecture Notes in Math., vol. 961, Springer, Berlin, 1982, pp. 314-491, DOI 10.1007/BFb0071291. MR708342 1307

[267] R. Thom, Ensembles et morphismes stratifiés (French), Bull. Amer. Math. Soc. 75 (1969), 240-284, DOI 10.1090/S0002-9904-1969-12138-5. MR0239613 1308

[268] M. Tibăr, Bouquet decomposition of the Milnor fibre, Topology 35 (1996), no. 1, 227-241, DOI 10.1016/0040-9383(95)00003-8. MR 1367282 298

[269] V. Turaev, Torsion invariants of Spin $^{c}$-structures on 3-manifolds, Math. Res. Lett. 4 (1997), no. 5, 679-695, DOI 10.4310/MRL.1997.v4.n5.a6. MR1484699 $\uparrow$

[270] I. Ustilovsky, Infinitely many contact structures on $S^{4 m+1}$, Internat. Math. Res. Notices 14 (1999), 781-791, DOI 10.1155/S1073792899000392. MR.1704176 倘12

[271] G. Valette, The link of the germ of a semi-algebraic metric space, Proc. Amer. Math. Soc. 135 (2007), no. 10, 3083-3090, DOI 10.1090/S0002-9939-07-08878-8. MR2322737 1309

[272] A. N. Varchenko, Zeta-function of monodromy and Newton's diagram, Invent. Math. 37 (1976), no. 3, 253-262, DOI 10.1007/BF01390323. MR0424806 1299

[273] A. N. Varčenko, Contact structures and isolated singularities (Russian, with English summary), Vestnik Moskov. Univ. Ser. I Mat. Mekh. 2 (1980), 18-21, 101. MR570582 1311

[274] J.-L. Verdier, Stratifications de Whitney et théorème de Bertini-Sard (French), Invent. Math. 36 (1976), 295-312, DOI 10.1007/BF01390015. MR0481096 295 308 318

[275] C. T. C. Wall, Stability, pencils and polytopes, Bull. London Math. Soc. 12 (1980), no. 6, 401-421, DOI 10.1112/blms/12.6.401. MR593960 334

[276] C. T. C. Wall, Singular points of plane curves, London Mathematical Society Student Texts, vol. 63, Cambridge University Press, Cambridge, 2004. MR2107253 1311 
[277] C. Weber (ed.) Noeuds, tresses et singularités. Comptes rendus du séminaire tenu aux Plans-sur-Bex (Suisse) en mars 1982. Monographie No.31 de L'Enseignement Mathématique (1983). 287

[278] H. Whitney, Tangents to an analytic variety, Ann. of Math. (2) 81 (1965), 496-549, DOI 10.2307/1970400. MR0192520 1308

[279] J. A. Wolf, Differentiable fibre spaces and mappings compatible with Riemannian metrics, Michigan Math. J. 11 (1964), 65-70. MR0159285 1292

[280] S. S. T. Yau, Hypersurface weighted dual graphs of normal singularities of surfaces, Amer. J. Math. 101 (1979), no. 4, 761-812, DOI 10.2307/2373918. MR.536041 \314

[281] O. Zariski, Studies in equisingularity. I. Equivalent singularities of plane algebroid curves, Amer. J. Math. 87 (1965), 507-536, DOI 10.2307/2373019. MR0177985 283

[282] O. Zariski, Studies in equisingularity. II. Equisingularity in codimension 1 (and characteristic zero), Amer. J. Math. 87 (1965), 972-1006, DOI 10.2307/2373257. MR0191898 1283. 307

[283] O. Zariski, Studies in equisingularity. III. Saturation of local rings and equisingularity, Amer. J. Math. 90 (1968), 961-1023, DOI 10.2307/2373492. MR0237493 283

[284] O. Zariski, Contributions to the problem of equisingularity, Questions on Algebraic Varieties (C.I.M.E., III Ciclo, Varenna, 1969), Edizioni Cremonese, Rome, 1970, pp. 261-343. MR 0276240 1283 307

[285] O. Zariski, Some open questions in the theory of singularities, Bull. Amer. Math. Soc. 77 (1971), 481-491, DOI 10.1090/S0002-9904-1971-12729-5. MR.0277533 1283 307

Instituto de Matemáticas, Universidad Nacional Autónoma de México, Cuidad UniVERSiTARIA, 04510 COYOACAN, MEXICO

Email address: jseade@im.unam.mx 